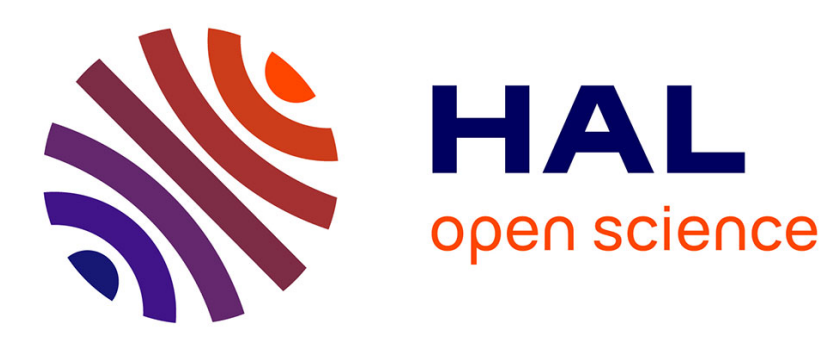

\title{
An uncountable family of pairwise non-Kakutani equivalent smooth diffeomorphisms
}

Mostapha Benhenda

\section{To cite this version:}

Mostapha Benhenda. An uncountable family of pairwise non-Kakutani equivalent smooth diffeomorphisms. 2013. hal-00795683

\section{HAL Id: hal-00795683 \\ https://hal.science/hal-00795683}

Preprint submitted on 1 Mar 2013

HAL is a multi-disciplinary open access archive for the deposit and dissemination of scientific research documents, whether they are published or not. The documents may come from teaching and research institutions in France or abroad, or from public or private research centers.
L'archive ouverte pluridisciplinaire HAL, est destinée au dépôt et à la diffusion de documents scientifiques de niveau recherche, publiés ou non, émanant des établissements d'enseignement et de recherche français ou étrangers, des laboratoires publics ou privés. 


\title{
An uncountable family of pairwise non-Kakutani equivalent smooth diffeomorphisms
}

\author{
Mostapha Benhenda*
}

February 27, 2013

\begin{abstract}
We construct an uncountable family of smooth ergodic zero-entropy diffeomorphisms that are pairwise non-Kakutani equivalent, on any smooth compact connected manifold of dimension greater than two, on which there exists an effective smooth circle action preserving a positive smooth volume. To that end, we first construct a smooth ergodic zero-entropy and non-Loosely Bernoulli diffeomorphism, by suitably modifying a smooth construction by Anosov and Katok. A construction of this kind was announced by Katok in 1977 and 1980 [8, p.141], [9, p.293].
\end{abstract}

\section{Introduction}

An important question on the interface between smooth dynamics and abstract ergodic theory is: what ergodic properties, if any, are imposed upon a dynamical system by the fact that it should be smooth? [11, p.89] [15, p.232] Only one restriction is known, which is that the entropy must be finite, because the dimension of the manifold is finite.

The core of the problem is when the invariant measure is smooth, and the manifold is compact (otherwise, see [10], [2]). No other restriction has been found yet, but examples have been provided: Brin, Feldman and Katok [5] showed that any compact manifold of dimension greater than one admits a smooth Bernoulli diffeomorphism. Katok [9] and Rudolph [14] gave examples of smooth non-Bernoulli K-diffeomorphisms. Ratner [13] showed that the Cartesian square of the horocycle flow is non-Loosely Bernoulli, thus giving an algebraic (hence analytic) example.

In this paper, we construct an uncountable family of smooth ergodic zero-entropy diffeomorphisms that are pairwise non-Kakutani equivalent, on any smooth compact connected manifold of dimension greater than two, on which there exists an effective smooth circle action preserving a positive smooth volume.

Our construction originates from an example given by Feldman [7], of an ergodic transformation of zero entropy that is non-Loosely Bernoulli (i.e. non-Kakutani equivalent to an irrational circle rotation). Ornstein, Rudolph and Weiss [12] extended Feldman's construction to obtain an uncountable family of ergodic zero-entropy transformations that are pairwise non-Kakutani equivalent. Their transformations are discontinuous, they are obtained by "cutting and stacking". The construction given in this

\footnotetext{
* Contact: mostaphabenhenda@gmail.com. I would like to thank Jean-Paul Thouvenot for communicating these questions, Sasha Prikhodko for discussion, and Poncelet Laboratory, Moscow, Russia, for hospitality.
} 
paper corresponds to a smooth version of the construction given by Ornstein, Rudolph and Weiss. To obtain it, we suitably modify a smooth construction by Anosov and Katok [1].

In particular, we also obtain a smooth ergodic non-Loosely Bernoulli diffeomorphism on any smooth compact connected manifold of dimension greater than two, on which there exists an effective smooth circle action preserving a positive smooth volume. This allows to generalize Katok's result on the existence of a smooth nonBernoulli K-diffeomorphism to every manifold of dimension greater than 4 [9, p.293]. This smooth version of Feldman's transformation has been announced by Katok [8, p.141], [9, p.293]. However, up to now, it has not been written.

In this paper, we show the theorems:

Theorem 1.1. Let $M$ be a smooth compact connected manifold of dimension $d \geqslant 2$, on which there exists an effective smooth circle action $\left(S_{t}\right)_{t \in \mathbb{T}^{1}}$ preserving a positive smooth measure $\mu$. There exists an ergodic non-Loosely Bernoulli diffeomorphism $T \in$ $\operatorname{Diff}^{\infty}(M, \mu)$.

Theorem 1.2. Let $M$ be a smooth compact connected manifold of dimension $d \geqslant 2$, on which there exists an effective smooth circle action $\left(S_{t}\right)_{t \in \mathbb{T}^{1}}$ preserving a positive smooth measure $\mu$. There exists an uncountable family of ergodic diffeomorphisms $T_{u} \in \operatorname{Diff}^{\infty}(M, \mu)$ such that if $u \neq v$, then $T_{u}$ and $T_{v}$ are not Kakutani-equivalent.

First, in sections 2 and 3, we show theorem 1.1, which gives a smooth version of Feldman's transformation. In section 4 , we show theorem 1.2 , by adapting the proof of Ornstein, Rudolph and Weiss [12, pp. 84-95].

\subsection{Main ideas}

We recall some definitions found in [7] (we slightly modify the terminology sometimes). We fix an automorphism $T$ of $(M, \mathcal{B}, \mu)$. Let $I$ be an alphabet of size $N$ and $P=\left\{c_{i}, i \in I\right\}$ a finite measurable partition indexed by this alphabet. For any $x \in M$ and integer $n \in \mathbb{N}$, and for any $i=0, \ldots, n-1$, let $a_{i} \in I$ such that $T^{i}(x) \in c_{a_{i}}$. The $n$-trajectory of $x$ by $T$ with respect to $P$ is the word of length $n$ on the alphabet $I$ given by: $a(T, n, x)=a_{0} \ldots a_{n-1}$, such that,$T^{i}(x) \in c_{a_{i}}$ (we do not mention the partition $P$ in the notation when it is fixed once for all). The length of this word is denoted $|a(T, n, x)|$. The trajectory of $x$ (by $T$ with respect to $P$ ) is the infinite word $a_{0} a_{1} \ldots$ where $T^{i}(x) \in c_{a_{i}}$ for any $i \in \mathbb{N}$.

Let $\alpha=a_{1} \ldots a_{n}$ and $\beta=b_{1} \ldots b_{m}$ be two words on the alphabet $I$. A match $\pi$ between $\alpha$ and $\beta$ is an injective, order-preserving partial function $\pi:\{1, \ldots, n\} \rightarrow\{1, \ldots, m\}$ such that for any $j$ in its domain of definition $\mathcal{D}(\pi) \subset\{1, \ldots, n\}, b_{\pi(j)}=a_{j}$. The cardinal of $\mathcal{D}(\pi)$ is denoted $|\mathcal{D}(\pi)|$. Let $\mathcal{R}(\pi)=\pi(\mathcal{D}(\pi)) . \pi$ is denoted:

$$
\pi: \mathcal{D}(\pi) \subset\{1, \ldots, n\} \rightarrow \mathcal{R}(\pi) \subset\{1, \ldots, m\}
$$

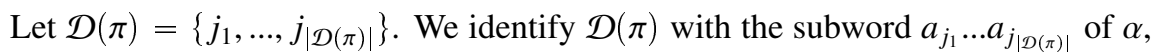
and $\mathcal{R}(\pi)$ with the subword $b_{\pi\left(j_{1}\right)} \ldots b_{\pi\left(j_{|\mathcal{D}(\pi)|}\right)}$ of $\beta$.

The fit of $\pi, \operatorname{fit}(\pi)$ is given by:

$$
\operatorname{fit}(\pi)=\frac{|\mathcal{D}(\pi)|}{\frac{1}{2}(m+n)}
$$

Let also 


$$
\pi^{-1}: \mathcal{R}(\pi) \subset\{1, \ldots, m\} \rightarrow \mathcal{D}(\pi) \subset\{1, \ldots, n\}
$$

such that $\pi^{-1}(\pi(i))=i$. Since $|\mathcal{R}(\pi)|=|\mathcal{D}(\pi)|$, then fit $\left(\pi^{-1}\right)=\operatorname{fit}(\pi)$.

The distance $\bar{f}(\alpha, \beta)$ between $\alpha$ and $\beta$ is:

$$
\bar{f}(\alpha, \beta)=1-\max \{\operatorname{fit}(\pi), \pi: \alpha \rightarrow \beta \text { match }\}
$$

Since fit $\left(\pi^{-1}\right)=\operatorname{fit}(\pi)$ for any match $\pi$, then $\bar{f}(\alpha, \beta)=\bar{f}(\beta, \alpha)$.

Moreover, if $\alpha, \beta$ and $\gamma$ are words of equal length, $\bar{f}(\alpha, \gamma) \leqslant \bar{f}(\alpha, \beta)+\bar{f}(\beta, \gamma)$.

A characterisation of Loosely Bernoulliness in the case of zero entropy is given in [7, p.22]. In this paper, we rather give a definition of non-Loosely Bernoulliness (nLB) in the case of zero entropy, because we want to obtain this property.

Definition 1.3. Suppose $T$ has no entropy. $T$ is non-Loosely Bernoulli $(n L B)$ if there exists $\epsilon>0$ and a finite partition $P$ such that, for an infinity of integers $n$, and for any $A \in \mathcal{B}$, if $\mu(A) \geqslant 1-\epsilon$, there exists $x, y \in A, \bar{f}(a(T, n, x), a(T, n, y)) \geqslant \epsilon$.

Our construction is inspired by the example given by Feldman [7] of a transformation $T_{0}$ that is ergodic, of zero entropy but non-Loosely Bernoulli. His transformation is not smooth (not even continuous) and is carried on $[0,1]$. He constructs words (of length $N(n)$ at the $n^{\text {th }}$ step) by induction. They are defined by $a_{0, i}=a_{i} \in I$ for $i=0, \ldots, N(0)-1$, and for $n \geqslant 0$ and $i=0, \ldots, N(n+1)-1$ :

$$
a_{n+1, i}=\left(a_{n, 1}^{N(n)^{2(i+1)}} \ldots a_{n, N(n)}^{N(n)^{2(i+1)}}\right)^{N(n)^{2(N(n+1)-(i+1))}}
$$

$T_{0}$ is constructed so that, up to minor details, the $N(n)$-trajectory of points in $[0,1]$ are, with equal Lebesgue measure, given by the $a_{n, i}, i=i, \ldots, N(n)$. Under suitable assumptions, this property implies that $T_{0}$ is nLB.

To get a construction looking like Feldman's, but smooth, we rely on three observations: first, the $\bar{f}$-distance is quite flexible: the fit of a match is a ratio of two lengths, and therefore, the addition of unknown letters into a word does not sensibly affect its fit with another word, if the total length of one of the words (or both) is taken sufficiently large. This property allows to approximate Feldman's map by smooth maps.

Second, we rely on a phenomenon of "quasi-concatenation" of finite trajectories: for example, let $q_{n}>0$, let $\zeta=\left\{[0,1] \times\left[i / q_{n},(i+1) / q_{n}\left[, 0 \leqslant i \leqslant q_{n}-1\right\}\right.\right.$ the partition of $[0,1] \times \mathbb{T}^{1}, S_{\frac{1}{q_{n}}}$ the rotation of angle $\frac{1}{q_{n}}$ of $[0,1] \times \mathbb{T}^{1}$. Let $w$ be the $q_{n}$-trajectory of 0 with respect to $S \frac{1}{q_{n}}$ and $\zeta$, and let $q_{n+1}>0$ be an integer that is strictly divided by $q_{n}^{2}$. Let $p_{n+1} / q_{n+1}=1 / q_{n}+1 / q_{n+1}$, and $\sigma$ be the circular permutation on words defined by: $\sigma: a_{1} a_{2} \ldots a_{p} \mapsto a_{2} \ldots a_{p} a_{1}$, where $a_{i}, i=1, \ldots, p$, are letters of a word of length $p$. The $q_{n+1}$-trajectory of 0 by $S_{\frac{1}{q_{n}}}$ is:

$$
w^{\frac{q_{n+1}}{q_{n}^{n}}}(\sigma(w))^{\frac{q_{n+1}}{q_{n}^{n}}}\left(\sigma^{q_{n}-1}(w)\right)^{\frac{q_{n+1}}{q_{n}^{n}}}
$$

We assume that $q_{n}^{2}$ divides $q_{n+1}$, and not simply that $q_{n}$ divides $q_{n+1}$, because the map $\sigma$ is applied every $q_{n+1} / q_{n}$ iterations, and for convenience, we prefer not to cut a word in the middle.

If $q_{n+1} / q_{n}$ is sufficiently large, we can neglect the effect of the circular permutation $\sigma$ on this trajectory, which fit becomes close to the fit of $w^{\frac{q_{n+1}}{q_{n}}}$. This phenomenon is used to smoothly "quasi-concatenate" words. 
The third observation allows to concatenate different words (the second observation only allows to concatenate the same word). It consists in introducing a smooth "quasipermutation" that allows to permute "tracks" on which rely the "trajectories" of points by our transformation $T$. By using quasi-permutations on separated tracks, we can obtain different trajectories, and thus obtain nLB. This method is possible because the manifold $M$ has dimension greater than two.

This technique of taking "different tracks" is a novelty with respect to the original Anosov-Katok method [1], which does not use dimension two as fully as we do. In their method, they only use one single "track". Basically, most of their construction can be carried on a circle. They need dimension two only when they take the limit in the construction. This approach complicates the coexistence of different trajectories on the same manifold: indeed, in their method, each trajectory is approximated by periodic trajectories. At step $n+1$, we need that the rotation $S_{\frac{p_{n+1}^{\prime}}{q_{n+1}^{\prime}}}$ of the annulus acts on a horizontal partition like a permutation having $N(n+1)=q_{n+1} / q_{n+1}^{\prime}$ cycles, each of length $q_{n+1}^{\prime}$. But the main problem is that the cycles are too closely intertwined (figure 1 ). This does not allow the convergence of the diffeomorphism $T_{n+1}=B_{n+1}^{-1} S_{\frac{p_{n+1}^{\prime}}{q_{n+1}^{\prime}}} B_{n+1}$ : the norm $\left\|B_{n+1}\right\|$ will be of order $q_{n+1}$ at least, whereas in order to get a smooth map at the limit, we need that the series $\sum_{n} \frac{\left\|B_{n+1}\right\|}{q_{n+1}^{\prime}}$ converges (it is a consequence of a generalized mean value theorem). In our construction, we put cycles vertically (figure 2), so that we do not get this problem.

However, we still rely on the core ideas of the Anosov-Katok method: we obtain the smooth diffeomorphism $T$ as the $C^{\infty}$-limit of a sequence $T_{n}=B_{n}^{-1} S \frac{p_{n}}{q_{n}} B_{n}$ of periodic diffeomorphisms, with $B_{n}=A_{n} A_{n-1} \ldots A_{1}, A_{n+1} S_{\frac{1}{q_{1}}}=S_{1} A_{n+1}$ and $q_{n}{ }^{q n}$ divides $q_{n+1}$.

Convergence in the $C^{\infty}$-norm is possible because $T_{n+1}^{q_{n}}$ is taken very close to $T_{n}$ : $q_{n+1}$ is taken large, so that the distance between $S_{\frac{p_{n+1}}{q_{n+1}}}$ and $S \frac{p_{n}}{q_{n}}$ is small with respect to the norm of the conjugacy $\left\|B_{n+1}\right\|$, which norm is related to scale of the smallest quasi-permutation at step $n+1$ (each quasi-permutation has its own scale, different of others, to allow nLB).

Moreover, in order to get $\mathrm{nLB}, T_{n+1}$ is also taken very close to $T_{n}$ : indeed, this closeness implies that $T_{n+1}^{i}$ does not significantly differ with $T_{n}^{i}$ for $i \sim q_{n}$, so that both transformations give similar $i$-trajectories. However, these two maps differ when $i>q_{n}$ (typically, when $i \sim q_{n+1} / q_{n}$ ). For example, $T_{n}$ is $q_{n}$-periodic but not $T_{n+1}$. This closeness allows approaching Feldman's construction: Feldman's maps $T_{n}$ and $T_{n+1}$ always have the same $N(n)$-trajectories, these two maps exactly coincide on increasingly larger sets. In our construction, for most points, there are $N(n+1)$ different kinds of $q_{n+1}$-trajectories, obtained by concatenating $q_{n}$-trajectories in different ways. Up to a circular permutation of letters, and up to other minor modifications, these $q_{n+1}$ trajectories are, for $i=0, \ldots, N(n+1)-1$ :

$$
a_{n+1, i}=\left(a_{n, 0}^{\frac{q_{n+1}}{N(n) q_{n, i} q_{n}}} a_{n, 1}^{\frac{q_{n+1}}{N(n) q_{n, i} q_{n}}} \ldots a_{n, N(n)-1}^{\frac{q_{n+1}}{N(n) q_{n, i} q_{n}}}\right)^{q_{n, i}}
$$

The parameters $q_{n, i}$ are suitably chosen to get nLB: an important characteristic of this choice is that $q_{n}<<q_{n, i}<<q_{n, i+1}<<\ldots<<q_{n+1}$.

In section 2 , we construct $T_{n}$ on $[0,1] \times \mathbb{T}$. In section 3 , we show that the limit $T$ is smooth, nLB and ergodic. In subsections 3.3.2 and 3.3.3, we extend the construction to the cases of $[0,1]^{d-1} \times \mathbb{T}$ and more general manifold $M$. In section 4 , we gener- 


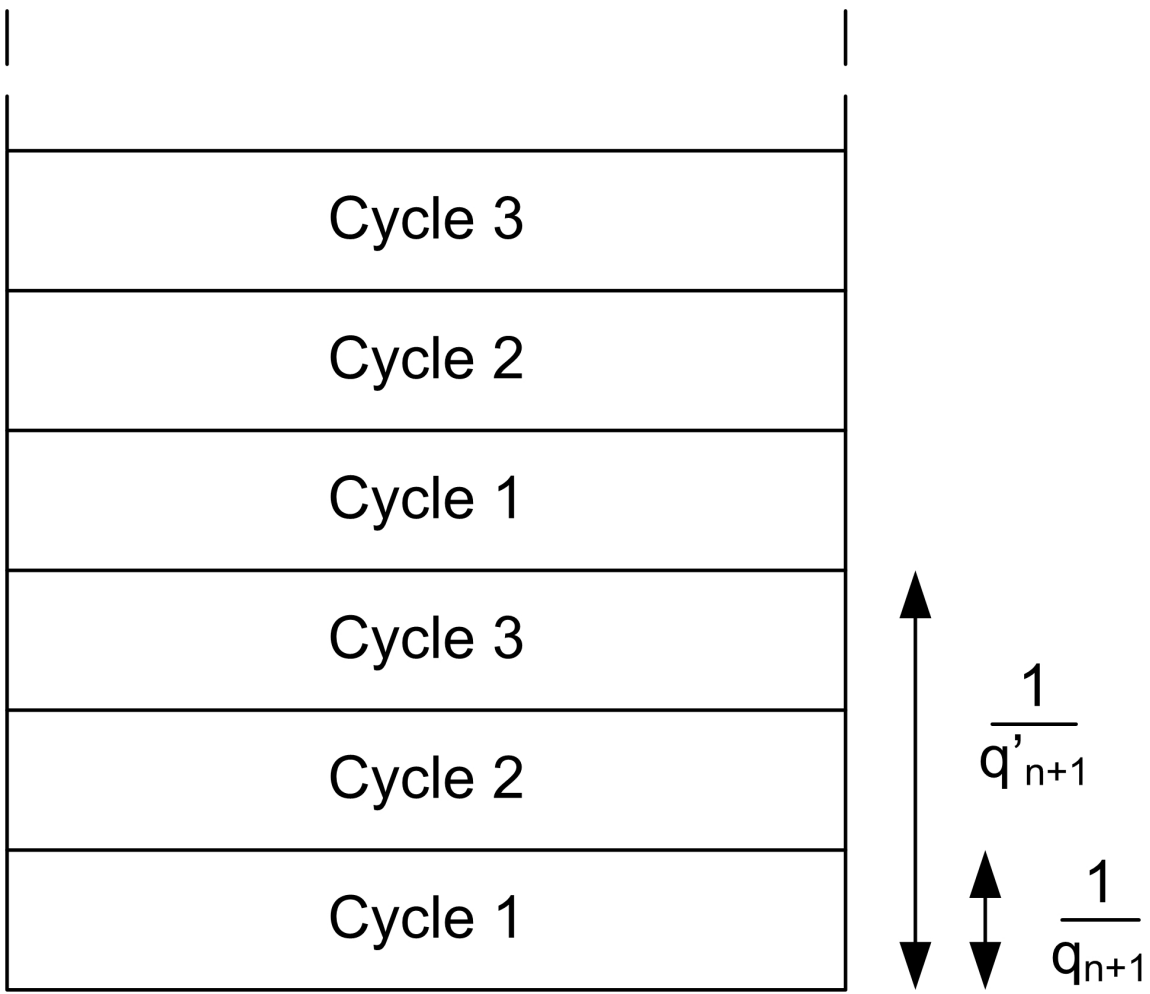

Figure 1: If we take a horizontal partition of $[0,1] \times \mathbb{T}^{1}$, the three cycles are too intertwined. This is an obstacle for the convergence of $T_{n}=B_{n}^{-1} S_{\frac{p_{n}^{\prime}}{q_{n}^{\prime}}} B_{n}$ towards a smooth map.

alize the construction to an uncountable family of pairwise non-Kakutani equivalent diffeomorphisms. Various figures illustrate the construction.

In all the paper, $f$ denotes an explicit function of its variables. The expression of this function can vary from one estimate to the other, but we still denote all these estimating functions in the same way.

\section{Construction of the transformation $T_{n}$}

\subsection{Smooth quasi-permutations}

We introduce smooth quasi-permutations, which are the main tool for the construction of the map $T$.

Proposition 2.1. Let $a<b$ and $c<d$ be real numbers such that $d-c<b-a$. Let $a<a^{\prime}<(a+b) / 2, b^{\prime}=a+b-a^{\prime}, 0<\epsilon<\min \left(\left(a^{\prime}-a\right) / 2,(d-c) / 2\right)$. Let $\tau_{u}$ be the translation of vector $(u, 0)$. There exists a smooth measure-preserving map $\phi\left(a, b, c, d, a^{\prime}, \epsilon\right):[a, b] \times[c, d]$ and a fixed numerical function $f$ such that (see figure 3):

$$
\phi_{\mid[a, b] \times[c, d]-([a+\epsilon / 2, b-\epsilon / 2] \times[c+\epsilon / 2, d-\epsilon / 2])}=i d
$$




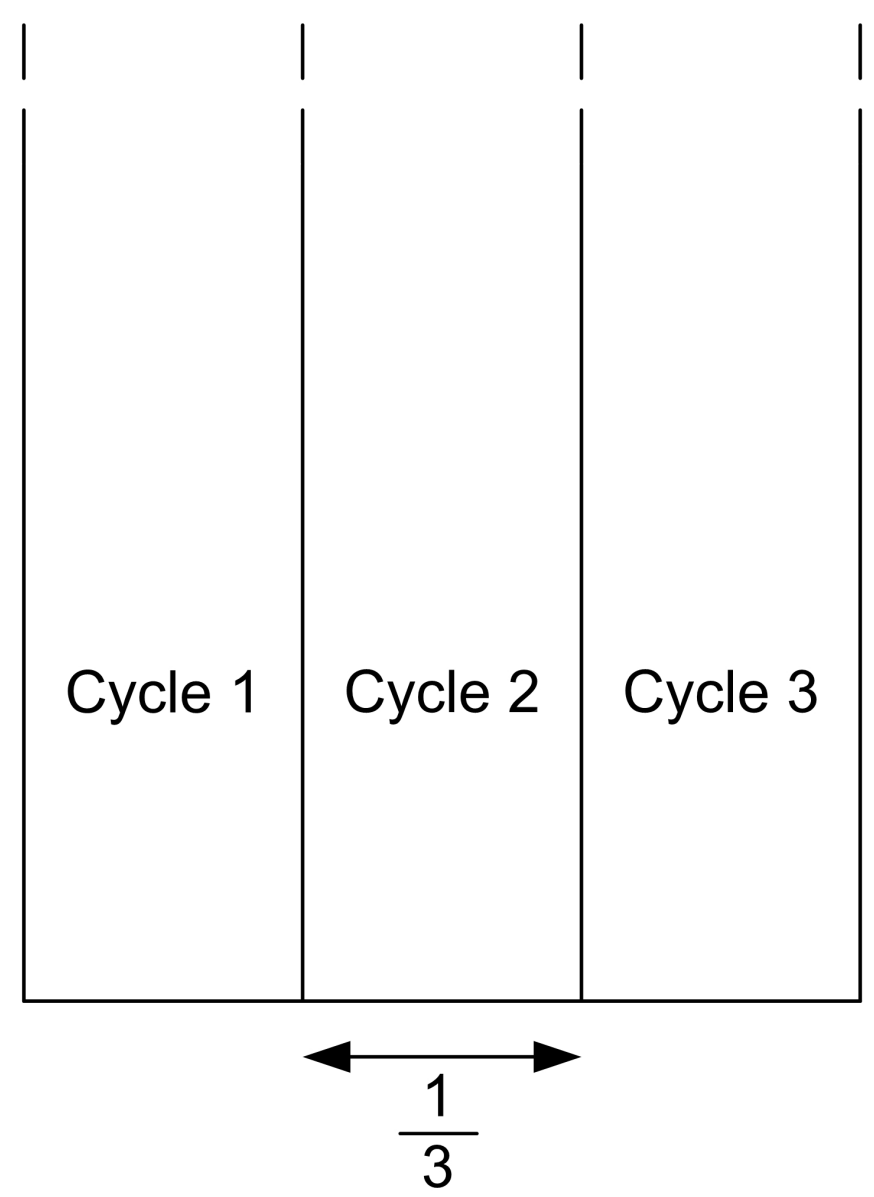

Figure 2: If we take a vertical partition of $[0,1] \times \mathbb{T}^{1}$, having to deal with three cycles is no longer an obstacle for the convergence of the periodic map $T_{n}=B_{n}^{-1} S_{\frac{p n}{q_{n}}} B_{n}$ towards a smooth map. 


$$
\begin{gathered}
\phi_{\mid\left[a^{\prime}+\epsilon, b^{\prime}-\epsilon\right] \times[c+\epsilon, d-\epsilon]}=i d \\
\phi_{\mid\left[a+\epsilon, a^{\prime}-\epsilon\right] \times[c+\epsilon, d-\epsilon]}=\tau_{b^{\prime}-a} \\
\phi_{\mid\left[b^{\prime}+\epsilon, b-\epsilon\right] \times[c+\epsilon, d-\epsilon]}=\tau_{a-b^{\prime}} \\
\|\phi\|_{n} \leqslant f\left(n, a, b, c, d, a^{\prime}, \epsilon\right)
\end{gathered}
$$

Remark 2.2. Assumptions given in this proposition are not the most general in order to define a quasi-permutation. However, they will be easier to write the construction, allowing to avoid writing some unessential technical details.

Remark 2.3. The estimating function $f\left(n, a, b, c, d, a^{\prime}, \epsilon\right)$ could be explicitly determined, but we do not need its expression in this paper.

The norm of the $C^{n}$-norm of $\phi$ is denoted with $\|\phi\|_{n}$. The set where $\|D \phi\|_{n}=1$, which includes $\left[a^{\prime}+\epsilon, b^{\prime}-\epsilon\right] \times[c+\epsilon, d-\epsilon] \cup\left[a+\epsilon, a^{\prime}-\epsilon\right] \times[c+\epsilon, d-\epsilon] \cup\left[b^{\prime}+\right.$ $\epsilon, b-\epsilon] \times[c+\epsilon, d-\epsilon] \cup[a, b] \times[c, d]-([a+\epsilon / 2, b-\epsilon / 2] \times[c+\epsilon / 2, d-\epsilon / 2])$ is called safe zone. The set where $\|\phi\|_{n} \neq 1$ is called turbulence zone.

The basic phenomenon that we use is the following: let $S_{t}$ be the rotation flow on A, $0<a<b<1,0<c<d<1, x=(u, v)$. We have: $S_{t}(u, v)=(u, v+t)$.

Let $A: \mathbb{A} \bigcirc$ such that $A_{\mid[a, b] \times[c, d]}=\phi\left(a, b, c, d, a^{\prime}, \epsilon\right)$, where $\phi\left(a, b, c, d, a^{\prime}, \epsilon\right)$ is the quasi-permutation defined in proposition 2.1, and such that $A=i d$ elsewhere. Let $R(x)=A^{-1} S_{t} A(x)$. We have:

1. If $u \notin[a, b]$, then $R(x)=S_{t}(x)$.

2. If $u \in\left[a+\epsilon, a^{\prime}-\epsilon\right], v \leqslant c$ and $c+\epsilon \leqslant v+t \leqslant d-\epsilon$, then $R(x)=\tau_{b^{\prime}-a} \circ S_{t}(x)$.

3. If $u \in\left[b^{\prime}+\epsilon, b-\epsilon\right], v \leqslant c$ and $c+\epsilon \leqslant v+t \leqslant d-\epsilon$, then $R(x)=\tau_{a-b^{\prime}} \circ S_{t}(x)$.

4. If $u \in\left[a^{\prime}+\epsilon, b^{\prime}-\epsilon\right], v \leqslant c$ and $c+\epsilon \leqslant v+t \leqslant d-\epsilon$, then $R(x)=S_{t}(x)$.

Observe also that, since $R^{2}(x)=A^{-1} S_{t} A A^{-1} S_{t} A(x)=A^{-1} S_{2 t} A(x)$, then if we take $x$ inside a safe zone, and if some iterate of $x$ by $R$ falls inside the turbulence zone, then if we iterate enough by $R$, we get back into a safe zone, as if we went there directly, i.e. as if we never crossed turbulences. So even if we lose the trajectory of $x$ into a turbulence zone, we recover it after sufficient iterations. Therefore, if turbulence zones are sufficiently thin, we can control most of the trajectory of $x$.

On the other hand, if $x$ belongs to a turbulence zone, we cannot control its trajectory by $R$.

Proof of proposition 2.1. We recall the following proposition, which is found in $[6,4$, $3]$ in a slightly modified version:

Proposition 2.4 ([6]). For any $\eta>0$, there exists a smooth measure-preserving map $\phi(\eta):[0,1]^{2} \bigcirc$ such that:

$$
\phi(\eta)_{\mid[\eta, 1-\eta]^{2}}=R(\pi,(1 / 2,1 / 2))
$$




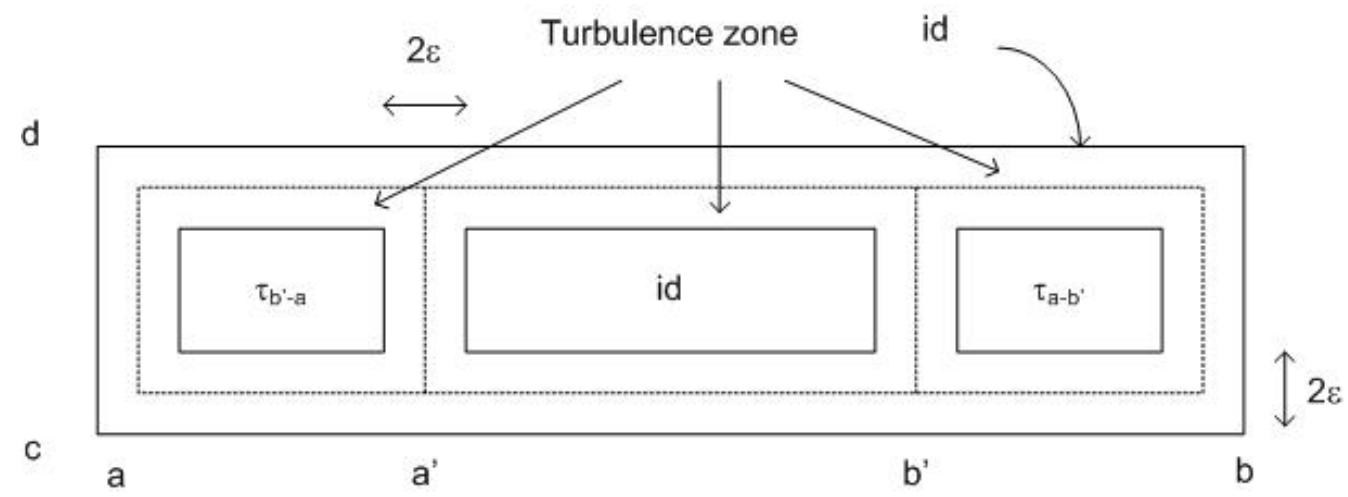

Figure 3: A quasi-permutation: it permutes the two lateral boxes, and keeps the central one fixed.

where $R(\pi,(1 / 2,1 / 2))$ denotes the rotation of angle $\pi$ and center $(1 / 2,1 / 2)$,

$$
\begin{gathered}
\phi(\eta)_{\mid[0,1]^{2}-\left([\eta / 2,1-\eta / 2]^{2}\right)}=i d \\
\|\phi(\eta)\|_{n} \leqslant f(n, \eta)
\end{gathered}
$$

Remark 2.5. In $[6,4,3]$, the angle of the rotation is $\pi / 2$, whereas the angle here is $\pi$.

Let $p \geqslant 1$ and

$$
\begin{aligned}
C_{p}:[0,1] \times\left[0, \frac{1}{p}\right] & \rightarrow[0,1] \times[0,1] \\
(x, y) & \mapsto(x, p y)
\end{aligned}
$$

Let $\phi(\eta, p)=C_{p}^{-1} \phi(\eta) C_{p}$. The map $\phi(\eta, p):[0,1] \times\left[0, \frac{1}{p}\right] \bigcirc$ is smooth and measure-preserving. By the Faa-di-Bruno formula,

$$
\|\phi(\eta, p)\|_{n} \leqslant f(n, \eta, p)
$$

By composing $\phi(\eta, p)$ with translations and homotheties, there exists $\phi^{1}(a, b, c, d, \epsilon)$ : $[a, b] \times[c, d] \bigcirc$ such that:

$$
\begin{gathered}
\phi_{\mid[a, b] \times[c, d]-([a+\epsilon / 2, b-\epsilon / 2] \times[c+\epsilon / 2, d-\epsilon / 2])}^{1}=i d \\
\phi_{\mid[a+\epsilon / 2, b-\epsilon / 2] \times[c+\epsilon / 2, d-\epsilon / 2]}^{1}=R\left(\pi,\left(\frac{a+b}{2}, \frac{c+d}{2}\right)\right) \\
\left\|\phi^{1}\right\|_{n} \leqslant f(n, a, b, c, d, \epsilon)
\end{gathered}
$$

there exists $\phi^{2}\left(a, b, c, d, a^{\prime}, \epsilon\right):[a, b] \times[c, d] \bigcirc$ such that

$$
\phi_{\mid\left[a^{\prime}+\epsilon, b^{\prime}-\epsilon\right] \times[c+\epsilon, d-\epsilon]}^{2}=R\left(\pi,\left(\frac{a+b}{2}, \frac{c+d}{2}\right)\right)
$$

and $\phi^{2}=i d$ on $[a, b] \times[c, d]-\left(\left[a^{\prime}, b^{\prime}\right] \times[c+\epsilon / 2, d-\epsilon / 2]\right)$

$$
\left\|\phi^{2}\right\|_{n} \leqslant f(n, a, b, c, d, \epsilon)
$$


there exists $\phi^{3}\left(a, b, c, d, a^{\prime}, \epsilon\right):[a, b] \times[c, d] \bigcirc$ such that

$$
\phi_{\left[\left[a+\epsilon, a^{\prime}-\epsilon\right] \times[c+\epsilon, d-\epsilon]\right.}^{3}=R\left(\pi,\left(\frac{a+a^{\prime}}{2}, \frac{c+d}{2}\right)\right)
$$

and $\phi^{3}=i d$ on $[a, b] \times[c, d]-\left(\left[a+\epsilon / 2, a^{\prime}\right] \times[c+\epsilon / 2, d-\epsilon / 2]\right)$

$$
\left\|\phi^{3}\right\|_{n} \leqslant f(n, a, b, c, d, \epsilon)
$$

and there exists $\phi^{4}\left(a, b, c, d, a^{\prime}, \epsilon\right):[a, b] \times[c, d] \bigcirc$ such that

$$
\phi_{\left[\left[b^{\prime}+\epsilon, b-\epsilon\right] \times[c+\epsilon, d-\epsilon]\right.}^{4}=R\left(\pi,\left(\frac{b+b^{\prime}}{2}, \frac{c+d}{2}\right)\right)
$$

and $\phi^{4}=i d$ on $[a, b] \times[c, d]-\left(\left[b^{\prime}, b\right] \times[c+\epsilon / 2, d-\epsilon / 2]\right)$

$$
\left\|\phi^{4}\right\|_{n} \leqslant f(n, a, b, c, d, \epsilon)
$$

We let $\phi=\phi^{4} \circ \phi^{3} \circ \phi^{2} \circ \phi^{1}$. We have:

$$
\begin{gathered}
\phi_{\mid[a, b] \times[c, d]-([a+\epsilon / 2, b-\epsilon / 2] \times[c+\epsilon / 2, d-\epsilon / 2])}=i d \\
\phi_{\mid\left[a^{\prime}+\epsilon, b^{\prime}-\epsilon\right] \times[c+\epsilon, d-\epsilon]}=i d \\
\phi_{\mid\left[a+\epsilon, a^{\prime}-\epsilon\right] \times[c+\epsilon, d-\epsilon]}=\tau_{b^{\prime}-a} \\
\phi_{\mid\left[b^{\prime}+\epsilon, b-\epsilon\right] \times[c+\epsilon, d-\epsilon]}=\tau_{a-b^{\prime}} \\
\|\phi\|_{n} \leqslant f\left(n, a, b, c, d, a^{\prime}, \epsilon\right)
\end{gathered}
$$

\subsection{Definition of $T_{n}$}

Figures 4,5 and 6 illustrate the definition. We define $T_{n}$ in the case $M=[0,1] \times \mathbb{T}$. In subsections 3.3.2 and 3.3.3, we extend the construction to the general case.

For $n \geqslant 0$, let $N(n) \geqslant 2$ be a sequence of integers. Additional assumptions on $N(n)$ appear in corollary 3.7 of the next section, to get nLB of the limit $T$. Let $N_{n}=$ $\prod_{k=0}^{n} N(k), N_{-1}=1$. Let strictly positive integers $q_{n}, q_{n, 0}, \ldots, q_{n, N(n+1)-1}$ such that $q_{n}$ divides $q_{n, 0}$, for $i=0, \ldots, N(n+1)-2, q_{n, i}$ divides $q_{n, i+1}$, and $N(n) q_{n} q_{n, N(n+1)-1}$ divides $q_{n+1}$. Additional assumptions on the $q_{n, i}$ appear in corollary 3.7, in order to get $\mathrm{nLB}$ of the limit $T$.

For $i=0, \ldots, N(n+1)-1, j=0, \ldots, N_{n-1}-1, j^{\prime}=0, \ldots, N_{n-1}-1, y=0, \ldots, N(n)-1$, $x=0, \ldots,\lfloor(N(n)+y) / 2\rfloor$, if $\left(j+j^{\prime}\right) / N_{n-1}<1$ then we define (by using notations of proposition 2.1):

$$
\begin{gathered}
A_{n+1}\left(i, j, j^{\prime}, x, y\right):\left(\frac{j}{N_{n-1}}+\frac{i}{N_{n+1}}+\left[\frac{x}{N_{n}}, \frac{N(n)-x+y}{N_{n}}+\frac{1}{N_{n+1}}+\frac{j^{\prime}}{N_{n-1}}\right]\right) \times \\
\left(\frac{j^{\prime}}{q_{n, i} N_{n}}+\left[\frac{y}{q_{n, i} N(n)}, \frac{y}{q_{n, i} N(n)}+\frac{1}{q_{n, i} N_{n}}\right]\right) \circlearrowright
\end{gathered}
$$




$$
\begin{gathered}
(u, v) \mapsto\left(\frac{j}{N_{n-1}}+\frac{i}{N_{n+1}}, \frac{j^{\prime}}{q_{n, i} N_{n}}\right)+ \\
\phi\left(\frac{x}{N_{n}}, \frac{N(n)-x+y}{N_{n}}+\frac{1}{N_{n+1}}+\frac{j^{\prime}}{N_{n-1}}, \frac{y}{q_{n, i} N(n)}, \frac{y}{q_{n, i} N(n)}+\frac{1}{q_{n, i} N_{n}}, \frac{x}{N_{n}}+\frac{1}{N_{n+1}}, \epsilon_{n+1}\right)(u, v)
\end{gathered}
$$

if $\left(j+j^{\prime}\right) / N_{n-1} \geqslant 1$ then we define (by using notations of proposition 2.1):

$$
\begin{gathered}
A_{n+1}\left(i, j, j^{\prime}, x, y\right):\left(\frac{j}{N_{n-1}}+\frac{i}{N_{n+1}}+\left[\frac{N(n)-x+y}{N_{n}}+\frac{j^{\prime}}{N_{n-1}}, \frac{x}{N_{n}}+\frac{1}{N_{n+1}}\right]\right) \times \\
\left(\frac{j^{\prime}}{q_{n, i} N_{n}}+\left[\frac{y}{q_{n, i} N(n)}, \frac{y}{q_{n, i} N(n)}+\frac{1}{q_{n, i} N_{n}}\right]\right) \circlearrowright \\
(u, v) \mapsto\left(\frac{j}{N_{n-1}}+\frac{i}{N_{n+1}}, \frac{j^{\prime}}{q_{n, i} N_{n}}\right)+
\end{gathered}
$$$$
\phi\left(\frac{N(n)-x+y}{N_{n}}, \frac{x}{N_{n}}+\frac{1}{N_{n+1}}+\frac{j^{\prime}}{N_{n-1}}, \frac{y}{q_{n, i} N_{n}}, \frac{y}{q_{n, i} N(n)}+\frac{1}{q_{n, i} N_{n}}, \frac{N(n)-x+y}{N_{n}}+\frac{1}{N_{n+1}}, \epsilon_{n+1}\right)(u, v)
$$

We distinguish the cases $\left(j+j^{\prime}\right) / N_{n-1}<1$ and $\left(j+j^{\prime}\right) / N_{n-1} \geqslant 1$, because if $\left(j+j^{\prime}\right) / N_{n-1} \geqslant 1$, then $\frac{j}{N_{n-1}}+\frac{i}{N_{n+1}}+\frac{N(n)-x+y}{N_{n}}+\frac{1}{N_{n+1}}+\frac{j^{\prime}}{N_{n-1}}>1$.

We briefly explain the different roles played by the indices $i, j, j^{\prime}, x, y$ : the index $i$ is used to label the $N(n+1)$ different $q_{n+1}$-trajectories. The parameters $x, y$ serve to concatenate the $N(n)$ different $q_{n}$-trajectories in the right order and everywhere. The parameters $j, j^{\prime}$ serve to connect the different ergodic components of $T_{n}$ properly, to get ergodicity of the limit transformation $T$.

We extend $A_{n+1}\left(i, j, j^{\prime}, x, y\right)$ to

$$
\begin{gathered}
E(i)=\bigcup_{j=0}^{N_{n-1}-1} \bigcup_{j^{\prime}=0}^{N_{n-1}-j-1} \bigcup_{y=0}^{N(n)-1} \bigcup_{x=0}^{[(N(n)+y) / 2\rfloor} \\
\left(\frac{j}{N_{n-1}}+\frac{i}{N_{n+1}}+\left[\frac{x}{N_{n}}, \frac{N(n)-x+y}{N_{n}}+\frac{1}{N_{n+1}}+\frac{j^{\prime}}{N_{n-1}}\right]\right) \times\left(\frac{j^{\prime}}{q_{n, i} N_{n}}+\left[\frac{y}{q_{n, i} N(n)}, \frac{y}{q_{n, i} N(n)}+\frac{1}{q_{n, i} N_{n}}\right]\right) \\
\left(\frac{j}{N_{n-1}}+\frac{i}{N_{n+1}}+\left[\frac{N(n)-x+y}{N_{n}}+\frac{j^{\prime}}{N_{n-1}}, \frac{x}{N_{n}}+\frac{1}{N_{n+1}}\right]\right) \times\left(\frac{j^{\prime}}{q_{n, i} N_{n}}+\left[\frac{y}{q_{n, i} N(n)}, \frac{y}{q_{n, i} N(n)}+\frac{1}{q_{n, i} N_{n}}\right]\right)
\end{gathered}
$$

by identity. Then, on each $E(i), i=0, . ., N(n+1)-1$, we define:

$$
A_{n+1}(i)=\circ_{j=0}^{N_{n-1}-1} \mathrm{O}_{j^{\prime}=0}^{N_{n-1}-1} \mathrm{o}_{y=0}^{N(n)-1} \mathrm{o}_{x=0}^{\lfloor(N(n)+y) / 2\rfloor} A_{n+1}\left(i, j, j^{\prime}, x, y\right)
$$

The order in which we compose the maps $A_{n+1}\left(i, j, j^{\prime}, x, y\right)$ affects the definition of $A_{n+1}(i)$, because their support are not disjoint: their turbulence zones have intersections 
on sets of small measure. However, this order does not matter for the properties of $A_{n+1}(i)$ that we seek.

We extend $A_{n+1}(i)$ to $E(i)+\left(0, l / q_{n, i}\right), l=0, \ldots, q_{n, i}-1$ by $1 / q_{n, i}$-equivariance, i.e.:

$$
A_{n+1}(i)\left(u, v+l / q_{n, i}\right)=A_{n+1}(i)(u, v)+\left(0, l / q_{n, i}\right)
$$

The parameter $i$ was sorted out because the $q_{n, i}$ depend on $i$.

Finally, on $[0,1] \times \mathbb{T}$, we define: $A_{n+1}=0_{i=0}^{N_{n-1}-1} A_{n+1}(i)$.

Again, the order of composition of the maps $A_{n+1}(i)$ matters for the definition, but not for the properties that we seek.

Let $B_{n}=A_{n} \circ \ldots \circ A_{0}$ and $T_{n}=B_{n}^{-1} S \frac{p_{n}}{q_{n}} B_{n}$. This defines $T_{n}$. In corollary 3.7, we add assumptions on $q_{n}, N(n)$ and $q_{n, i}$ to obtain that $T_{n}$ converges towards a smooth, nLB and ergodic transformation $T$. $T$ will have zero entropy as the limit of maps conjugated to rotations.

\section{Properties of the transformation $T$}

\subsection{Convergence of $T_{n}$ towards a smooth map $T$}

Showing the convergence of $T_{n}$ towards a smooth map $T$ is classical (see e.g. [1, 6]). By construction, there exists $f_{\text {precgce }}\left(n, q_{n}, N_{n+1}, q_{n, N(n+1)-1}, \epsilon_{n+1}\right)$ such that $\left\|B_{n+1}\right\|_{n+1} \leqslant$ $f_{\text {precgce }}\left(n, q_{n}, N_{n+1}, q_{n, N(n+1)-1}, \epsilon_{n+1}\right)$. By the Cauchy criterion, it suffices to show that $\sum_{n \geqslant 0} d_{n}\left(T_{n+1}, T_{n}\right)$ converges. We combine the fact that $A_{n+1}$ commutes with $S_{\frac{1}{q n}}$, the estimation of $B_{n+1}$ and the fact that $p_{n+1} / q_{n+1}=p_{n} / q_{n}+1 / q_{n+1}$. We recall the lemma [6, p.1812]:

Lemma 3.1. Let $k \in \mathbb{N}$. There is a constant $C(k, d)$ such that, for any $h \in \operatorname{Diff}(M)$, $\alpha_{1}, \alpha_{2} \in \mathbb{R}$, we have:

$$
d_{k}\left(h S_{\alpha_{1}} h^{-1}, h S_{\alpha_{2}} h^{-1}\right) \leqslant C(k, d)\|h\|_{k+1}^{k+1}\left|\alpha_{1}-\alpha_{2}\right|
$$

Since $T_{n}=B_{n}^{-1} S_{\frac{p_{n}}{q_{n}}} B_{n}=B_{n+1}^{-1} S_{\frac{p_{n}}{q_{n}}} B_{n+1}$ (because $A_{n+1}$ commutes with $S_{\frac{1}{q_{n}}}$ ), and since, for $n \geqslant 2,\left\|\phi_{n}\right\|_{n+1} \leqslant q_{n}^{R_{1}(n)}$ for a sequence $R_{1}(n)$ independent of $q_{n}$ (because $q_{n} \geqslant 2$ for $\left.n \geqslant 2\right)$, we obtain, for a fixed sequence $f_{c g c e}\left(n, q_{n}, N_{n+1}, q_{n, N(n+1)-1}, \epsilon_{n+1}\right)$ :

$$
\begin{gathered}
d_{n}\left(T_{n+1}, T_{n}\right)=d_{n}\left(B_{n+1}^{-1} S_{\frac{p_{n+1}}{q_{n+1}}} B_{n+1}, B_{n+1}^{-1} S_{\frac{p_{n}}{q_{n}}} B_{n+1}\right) \\
\leqslant C(k, d)\left\|B_{n+1}\right\|_{n+1}^{n+1}\left|\frac{p_{n+1}}{q_{n+1}}-\frac{p_{n}}{q_{n}}\right| \leqslant f_{c g c e}\left(n, q_{n}, N_{n+1}, q_{n, N(n+1)-1}, \epsilon_{n+1}\right)\left|\frac{p_{n+1}}{q_{n+1}}-\frac{p_{n}}{q_{n}}\right|
\end{gathered}
$$

For a sufficiently increasing sequence $q_{n}$, this last estimate guarantees the convergence of $T_{n}$ in the smooth topology. 


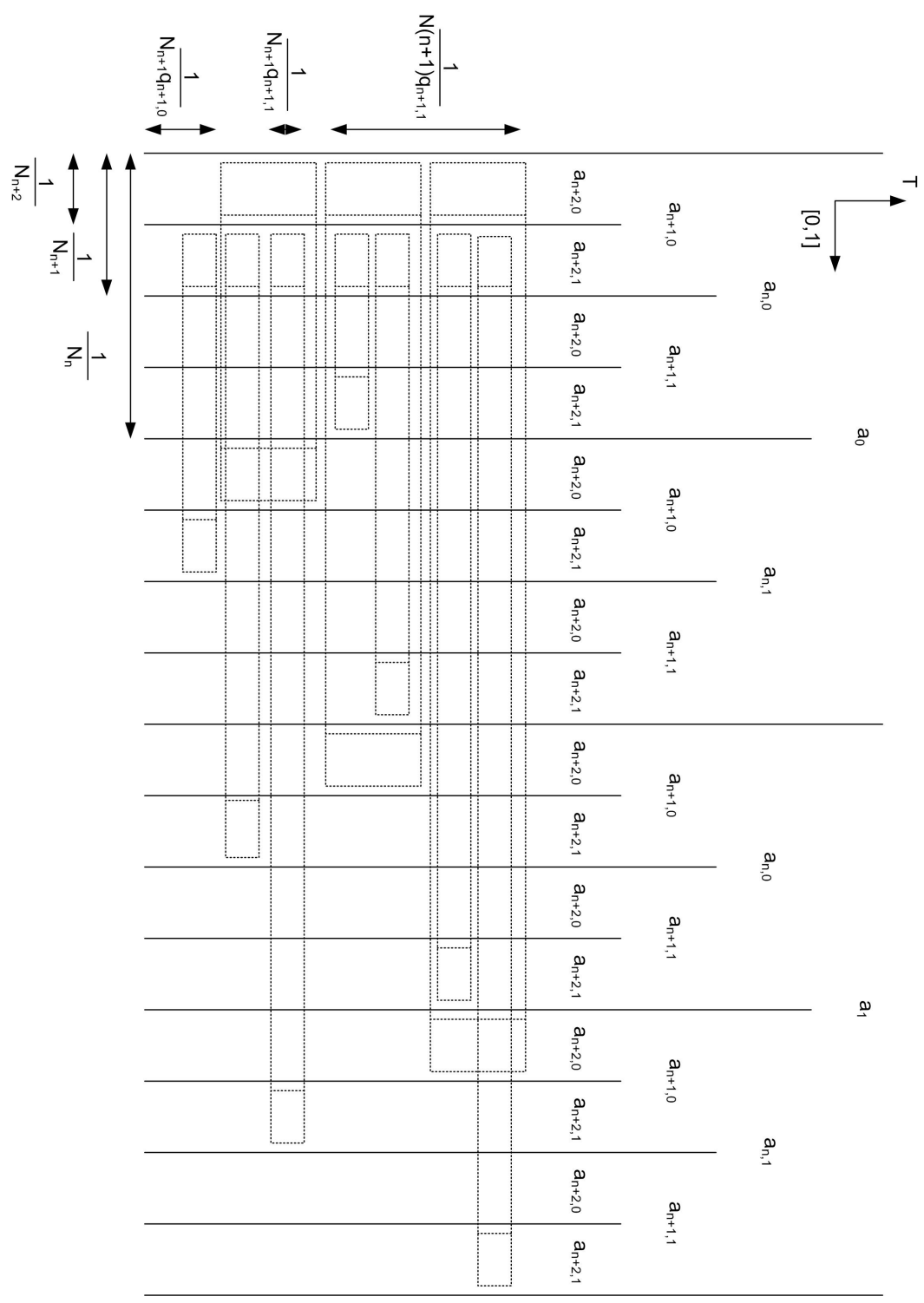

Figure 4: Domains of the different trajectories for the first three iterations of the construction, with $N(k)=2, k=0, \ldots, 3$. Some quasi-permutations are represented in dotted lines. 


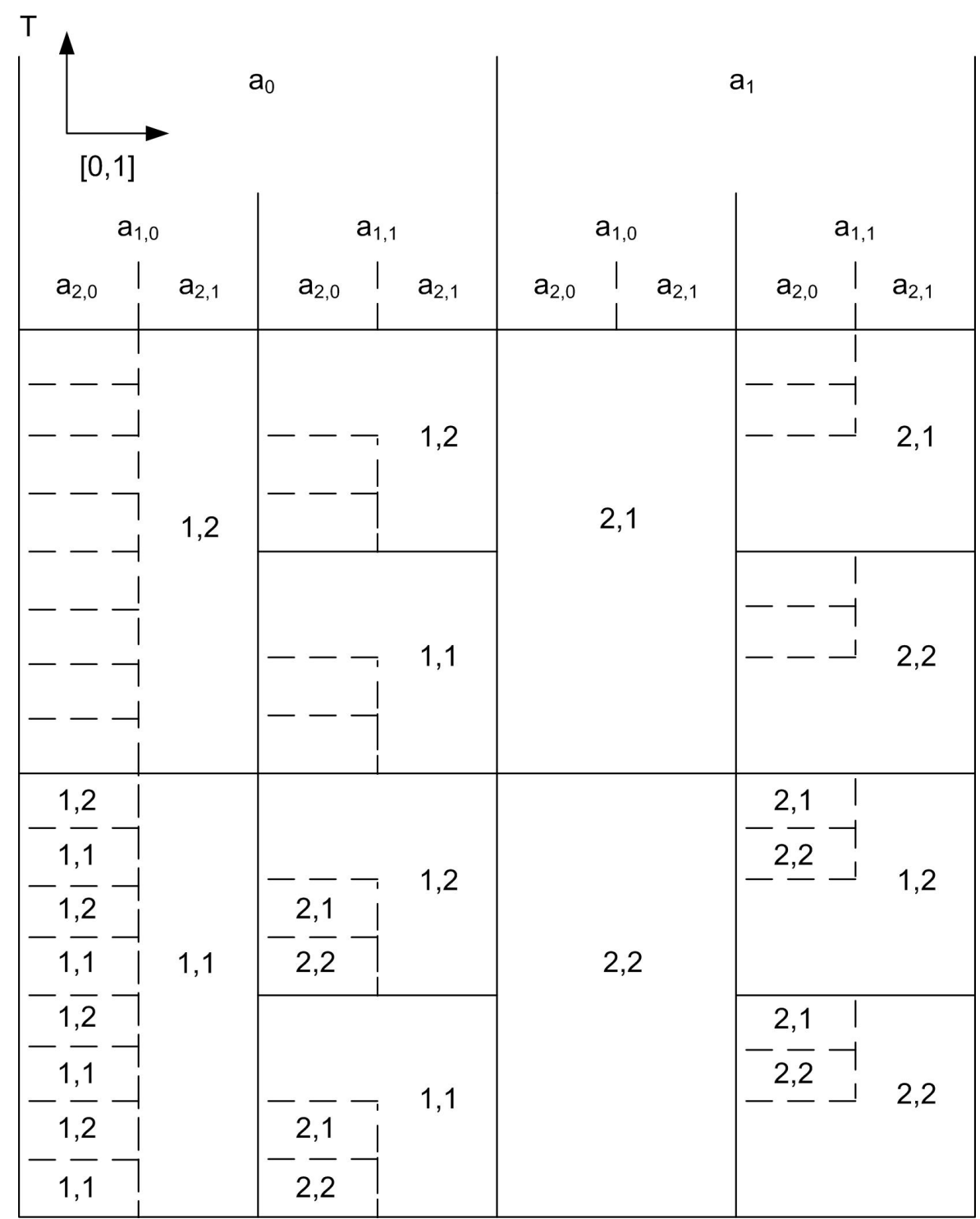

Figure 5: Representation of $A_{1}$ and partial representation of $A_{2}$ (dashed lines), with $N(k)=2, k=0, \ldots, 2$. The rectangle $(i, j)$ is quasi-permuted with the rectangle $(j, i)$ of the same height. In particular, $A_{l}(l=1,2)$ is the identity on $(i, i)$. 

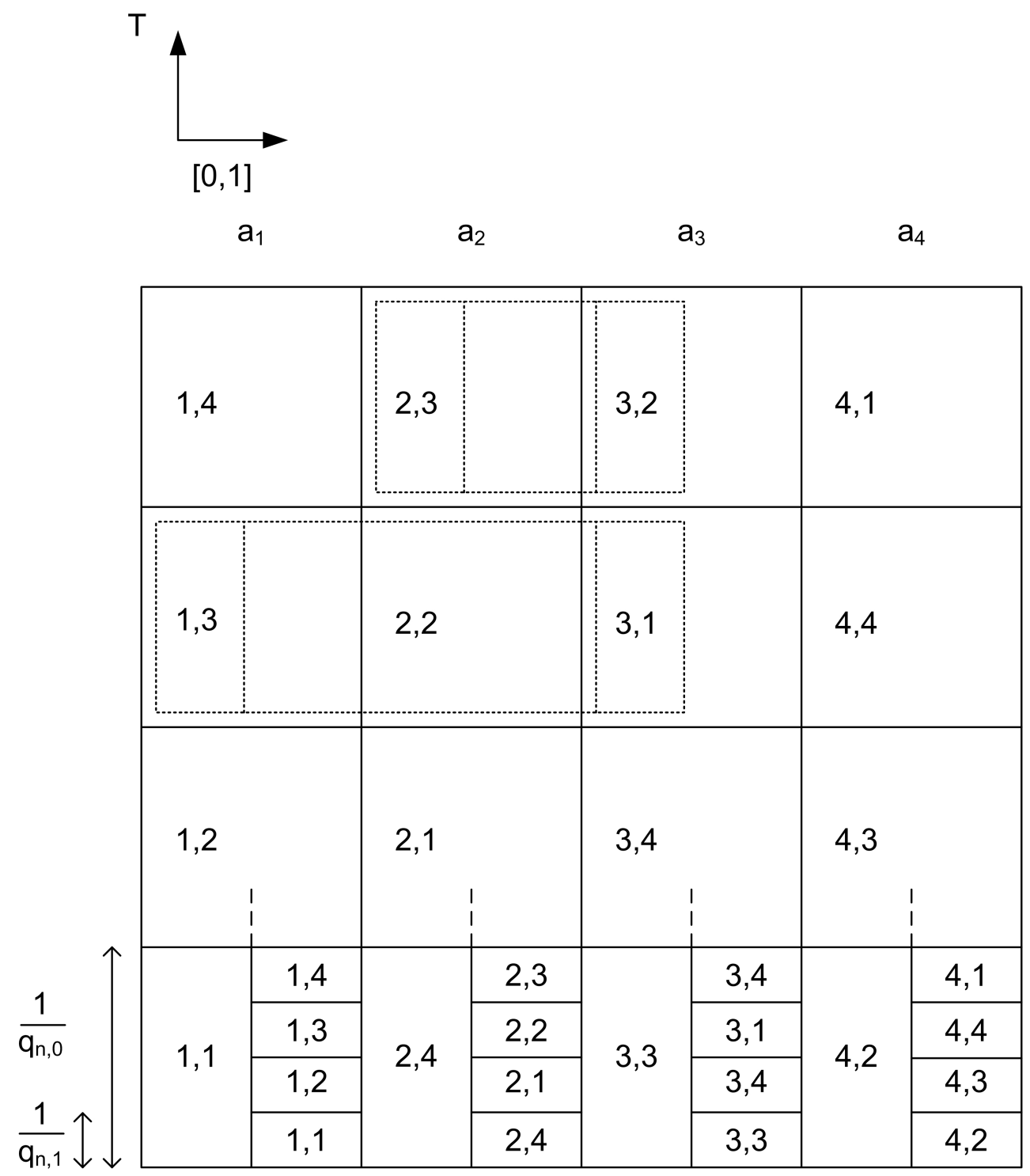

Figure 6: Partial representation of $A_{1}$, with $N(0)=4$ and $N(1)=2$. The rectangle $(i, j)$ is quasi-permuted with the rectangle $(j, i)$ of the same height. Some quasipermutations are represented in dotted lines. 


\section{2 $T$ is non-Loosely Bernoulli}

To get nLB, the idea is that the two words aaaabbbb and $a b a b a b a b$ are far from each other in the $\bar{f}$-distance.

We fix the partition: $\left.\left.P=\left\{c_{j}=\right] \frac{j}{N(0)}, \frac{j+1}{N(0)}\right] \times \mathbb{T}^{1}, j=0, \ldots, N(0)-1\right\}$

The aim of this subsection is to show the following proposition, which is slightly stronger than the $\mathrm{nLB}$ property:

Proposition 3.2. For any $\epsilon>0$, there exists $T \in \operatorname{Diff}^{\infty}(\mathbb{A})$ such that for any $A \in \mathcal{B}$ such that $\mu(A)>2 \epsilon$, there exists $n_{0} \geqslant 0$ such that for any $n \geqslant n_{0}$, there exists $x, y \in A$ such that $\bar{f}\left(a\left(T, q_{n}, x\right), a\left(T, q_{n}, y\right)\right) \geqslant 1-3 \epsilon$.

First, we show that it suffices to consider $q_{n}$-trajectories by $T_{n}$, instead of considering $q_{n}$-trajectories by $T$.

Lemma 3.3. For any $\epsilon>0$, there exists a numerical map $f_{n l b}\left(\epsilon, n, q_{n}, N_{n+1}, q_{n, N(n+1)-1}, \epsilon_{n+1}\right) \geqslant$ $f_{\text {cgce }}\left(n, q_{n}, N_{n+1}, q_{n, N(n+1)-1}, \epsilon_{n+1}\right)$, there exists $E_{n l b} \subset \mathbb{A}$ such that $\mu\left(E_{n l b}\right) \geqslant 1-\epsilon$, and such that for any $n \in \mathbb{N}$, if $q_{n+1} \geqslant f_{n l b}\left(\epsilon, n, q_{n}, N_{n+1}, q_{n, N(n+1)-1}, \epsilon_{n+1}\right)$, then for any $x \in E_{n l b}$ the $q_{n}$-trajectory of $x$ by $T_{n}$ is the same as the $q_{n}$-trajectory of $x$ by $T$.

Proof. Let $f_{n l b}\left(\epsilon, n, q_{n}, N_{n+1}, q_{n, N(n+1)-1}, \epsilon_{n+1}\right) \geqslant f_{\text {cgce }}\left(n, q_{n}, N_{n+1}, q_{n, N(n+1)-1}, \epsilon_{n+1}\right) \geqslant$ $2^{n} q_{n}$ such that, if $q_{n+1} \geqslant f_{n l b}\left(\epsilon, n, q_{n}, N_{n+1}, q_{n, N(n+1)-1}, \epsilon_{n+1}\right)$, then for any $m \in \mathbb{N}$,

$$
\sum_{n \geqslant m} \frac{f_{\text {cgce }}\left(n, q_{n}, N_{n+1}, q_{n, N(n+1)-1}, \epsilon_{n+1}\right)}{q_{n+1}} \leqslant \frac{\epsilon}{q_{m}^{2} N(0) 2^{m+1}}
$$

Remark that as far as $q_{n+1} \geqslant 2^{n} q_{n}, f_{n l b}$ does not depend on the $q_{p}, p \geqslant n+1$. Since

$$
d\left(T, T_{m}\right) \leqslant \sum_{n \geqslant m} \frac{f_{\text {cgce }}\left(n, q_{n}, N_{n+1}, q_{n, N(n+1)-1}, \epsilon_{n+1}\right)}{q_{n+1}}
$$

then

$$
d\left(T, T_{m}\right) \leqslant \frac{\epsilon}{q_{m}^{2} N(0) 2^{m+1}}
$$

Therefore, for $0 \leqslant i \leqslant q_{n}-1$,

$$
d_{0}\left(T^{i}, T_{m}^{i}\right) \leqslant \frac{\epsilon}{q_{m} N(0) 2^{m+1}}
$$

Moreover, for any $F, G$ continuous and measure-preserving transformations, and $A \in \mathcal{B}$,

$$
\mu(F(A) \cap G(A)) \geqslant \mu(A)-\mu[F(A) \bar{f} G(A)] \geqslant \mu(A)-d_{0}(F, G)
$$

Now, let

$$
E_{n l b}=\bigcap_{n \geqslant 0} \bigcap_{i=0}^{q_{n}-1}\left[\bigcup_{j=0}^{N(0)-1} T_{n}^{-i} c_{j} \cap T^{-i} c_{j}\right]
$$

Since, by (3),

$$
\mu\left(T_{n}^{-i} c_{j} \cap T^{-i} c_{j}\right) \geqslant \mu\left(c_{j}\right)-d\left(T_{n}^{-i}, T^{-i}\right)
$$


and since $T^{-i} c_{j} \cap T^{-i} c_{j^{\prime}}=\varnothing$ if $j \neq j^{\prime}$, then

$$
\begin{gathered}
\mu\left(\bigcup_{j=0}^{N(0)-1} T_{n}^{-i} c_{j} \cap T^{-i} c_{j}\right)=\sum_{j=0}^{N(0)-1} \mu\left(T_{n}^{-i} c_{j} \cap T^{-i} c_{j}\right) \\
\geqslant \sum_{j=0}^{N(0)-1} \mu\left(c_{j}\right)-d\left(T_{n}^{-i}, T^{-i}\right) \geqslant 1-\frac{\epsilon}{q_{n} 2^{n+1}}
\end{gathered}
$$

Moreover, for any $A, B \in \mathcal{B}, \epsilon_{A}, \epsilon_{B} \geqslant 0$ such that $\mu(A) \geqslant 1-\epsilon_{A}$ and $\mu(B) \geqslant 1-\epsilon_{B}$, we have:

$$
\mu(A \cap B)=\mu(A)+\mu(B)-\mu(A \cup B) \geqslant 1-\left(\epsilon_{A}+\epsilon_{B}\right)
$$

Therefore,

$$
\mu\left(E_{n l b}\right) \geqslant 1-\sum_{n \geqslant 0} \sum_{i=0}^{q_{n}-1} \frac{\epsilon}{q_{n} 2^{n+1}}=1-\epsilon
$$

Finally, if $x \in E_{n l b}$, then for any $n \in \mathbb{N}$, for any $i=0, \ldots, q_{n}-1$, there exists $j=0, \ldots, N(0)-1$ such that $x \in T_{n}^{-i} c_{j} \cap T^{-i} c_{j}$. Therefore, $T_{n}^{i} x \in c_{j}$ and $T^{i} x \in c_{j}$. Therefore, $x$ has the same $q_{n}$-trajectory by $T_{n}$ and by $T$.

Second, we show that the trajectory by $T_{n}$ of most points is well approximated by the "theoretical" trajectories $a_{n, i}$, defined in (1).

Let $\eta_{0}=0$, and for any $n \geqslant 1$, let

$\eta_{n}=2 \sum_{k=0}^{n-1} \epsilon_{k+1} N_{k+1} \max _{0 \leqslant i \leqslant N(k+1)-1} q_{k, i}+\frac{1}{\min _{0 \leqslant i \leqslant N(k+1)-1} q_{k, i}}+\max _{0 \leqslant i \leqslant N(k+1)-1} \frac{N(k)^{2} q_{k}^{2} q_{k, i}}{q_{k+1}}$

and

$$
E_{\text {safe }}(n)=\bigcap_{k=0}^{n} \operatorname{safe}\left(A_{k}\right)
$$

We have the lemma:

Lemma 3.4. We have a partition $E_{\text {safe }}(n)=\left\{c_{0}(n), \ldots, c_{N(n)-1}(n)\right\}$ such that for any $i=0, \ldots, N(n)-1$,

$$
\left|\mu\left(c_{i}(n)\right)-\frac{1}{N(n)}\right| \leqslant \mu\left(\operatorname{turb}\left(B_{n}\right)\right)
$$

and for any $x \in c_{i}(n)$

$$
\bar{f}\left(a\left(T_{n}, q_{n}, x\right), a_{n, i}\right) \leqslant \eta_{n}
$$

Proof. The proof is by induction on $n$. If $n=0, E_{\text {safe }}(0)=\mathbb{A}$. Moreover, $a\left(T_{0}, q_{0}, x\right)=$ $i_{x}$, where $i_{x} \in I$ is such that $x \in c_{i_{x}}$. Therefore, $a\left(T_{0}, q_{0}, x\right)=a_{0, i_{x}}$, and $\bar{f}\left(a\left(T_{0}, q_{0}, x\right), a_{0, i_{x}}\right) \leqslant$ $\eta_{0}=0$.

Suppose the lemma holds at step $n$, and let $x \in E_{\text {safe }}(n+1)$. Since $\operatorname{safe}\left(A_{n}\right)$ is stable by $A_{n}$, then $E_{\text {safe }}(n+1) \subset$ safe $\left(B_{n+1}\right)$, and therefore, $x \in$ safe $\left(B_{n+1}\right)$. By construction, up to a circular permutation, $x$ has $N(n+1)$ possible types of $q_{n+1}$-trajectories (i.e. $N(n+1)$ if we neglect turbulences, otherwise there are $N_{n+1}$ possible $q_{n+1}$-trajectories), 
depending on which "track" it stands. We denote these (non-connected) elements of this partition $c_{0}(n+1), \ldots, c_{N(n+1)-1}(n+1)$. We have:

$$
\left|\mu\left(c_{i}(n+1)\right)-\frac{1}{N(n+1)}\right| \leqslant \mu\left(\operatorname{turb}\left(B_{n+1}\right)\right)
$$

We show the second estimate. By construction, up to a circular permutation, any $y \in$ $E_{\text {safe }}(n) \subset$ safe $\left(B_{n}\right)$ has $N(n)$ possible types of $q_{n}$-trajectories by $T_{n}$. We denote them $a_{n, i, \text { eff }}$, with $i=0, \ldots, N(n)-1$. Labels $i$ of $a_{n, i, \text { eff }}$ are chosen such that, by induction assumption, $\bar{f}\left(a_{n, i, \text { eff }}, a_{n, i}\right) \leqslant \eta_{n} . a_{n, i, \text { eff }}$ is the "effective" trajectory: it corresponds to an "ideal" trajectory $a_{n, i}$ perturbed by turbulences coming from $B_{n}$. These turbulences depend on the point $y$, and for better precision, we could write $a_{n, i, \operatorname{eff}(y)}$.

First, we neglect turb $\left(A_{n+1}\right)$ (we suppose it infinitely thin). By construction, the $q_{n+1}$-trajectory of $x, a_{n+1, i, \text { neg }}$ (for $0 \leqslant i \leqslant N(n+1)-1$ ) is of the form:

$$
\begin{gathered}
a_{n+1, i, \text { negl }}=\sigma^{u}\left[\left(\left[\sigma^{l_{0}}\left(a_{n, 0, \text { eff }}\right)\right]^{\frac{q_{n+1}}{N(n) q_{n, i} q_{n}}} \ldots\left[\sigma^{l_{N(n)-1}}\left(a_{n, N(n)-1, \text { eff }}\right)\right]^{\frac{q_{n+1}}{N(n) q_{n, i} q_{n}}}\right)^{\frac{q_{n, i}}{q_{n}}} \ldots\right. \\
\left(\left[\sigma^{q_{n}-1+l_{0}}\left(a_{n, 0, \text { eff }}\right)\right]^{\frac{q_{n+1}}{N(n) q_{n, i} q_{n}}} \ldots\left[\sigma^{q_{n}-1+l_{N(n)-1}}\left(a_{n, N(n)-1, \mathrm{eff})}\right]^{\frac{q_{n+1}}{N(n) q_{n, i} q_{n}}}\right)^{\frac{q_{n, i}}{q_{n}}}\right]
\end{gathered}
$$

for some integers $u, l_{0}, \ldots, l_{N(n)-1}$. In particular, at $y$ fixed, there are only $N(n)$ possible words $a_{n, i, \mathrm{eff}(y)}, i=0, \ldots, N(n)-1$ that compose the $q_{n+1}$-trajectory of $y$ in the formula above. Turbulences coming from $B_{n}$ are the same in all these words. This fact is important for the construction of the uncountable family of pairwise non-Kakutani equivalent diffeomorphisms.

For all $i=0, \ldots, N(n+1)-1$, let also:

$$
a_{n+1, i, \text { semeff }}=\left(a_{n, 0, \text { eff }}^{\frac{q_{n+1}}{N(n) q_{n}, q_{n}}} a_{n, 1, \text { eff }}^{\frac{q_{n+1}}{N(n) q_{n, i} q_{n}}} \ldots a_{n, N(n)-1, \text { eff }}^{\frac{q_{n+1}}{N(n) q_{n} q_{n}}}\right)^{q_{n, i}}
$$

(the index "semeff" is for "semi-effective": $a_{n+1, i, \text { semeff }}$ is halfway between the "effective" trajectory $a_{n+1, i, \text { eff }}$ and the "ideal" trajectory $\left.a_{n+1, i}\right)$. Moreover, for any integer $N \geqslant 2$, integer $k$, and word $a, \sigma^{k}\left(a^{N}\right)=a^{\prime} a^{N-2} a^{\prime \prime}$, where $a^{\prime}$ and $a^{\prime \prime}$ are words such that $\left|a^{\prime}\right|+\left|a^{\prime \prime}\right|=|a|$. Therefore,

$$
\bar{f}\left(a^{N}, \sigma^{k}\left(a^{N}\right)\right) \leqslant 1-\frac{(N-2)|a|}{N|a|}=\frac{2}{N}
$$

Therefore,

$$
\bar{f}\left(a_{n+1, i, \text { neg }}, \sigma^{u}\left[a_{n+1, i \text {,semeff }}\right]\right) \leqslant \frac{2 N(n)^{2} q_{n}^{2} q_{n, i}}{q_{n+1}}
$$

Now, we take into account turb $\left(A_{n+1}\right)$. The $q_{n+1}$-trajectory of $x$ crosses turbulences from quasi-permutations making up its own trajectory, but also from quasipermutations making up other trajectories (see figure 4). Therefore, it crosses at most

$2 N_{n+1} \max _{0 \leqslant i \leqslant N(n+1)-1} q_{n, i}$ turbulence zones (the factor 2 is because we cross one turbulence zone to get in and another to get out), each of width $\epsilon_{n+1}$. Therefore, 


$$
\bar{f}\left(a\left(T_{n+1}, q_{n+1}, x\right), a_{n+1, i, \text { neg }}\right) \leqslant 2 \epsilon_{n+1} N_{n+1} \max _{0 \leqslant i \leqslant N(n+1)-1} q_{n, i}
$$

To conclude the proof, we also need the lemma:

Lemma 3.5. Let $a, b, a^{\prime}, b^{\prime}$ words such that $|a|=|b|=\left|a^{\prime}\right|=\left|b^{\prime}\right|$. We have:

$$
\bar{f}\left(a b, a^{\prime} b^{\prime}\right) \leqslant \frac{1}{2}\left(\bar{f}\left(a, a^{\prime}\right)+\bar{f}\left(b, b^{\prime}\right)\right)
$$

By applying lemma 3.5 and the induction assumption, for any integer $u$, we get:

$$
\bar{f}\left(\sigma^{u}\left(a_{n+1, i, \mathrm{Semeff}}\right), \sigma^{u}\left(a_{n+1, i}\right)\right)=\bar{f}\left(a_{n+1, i, \mathrm{Semeff}}, a_{n+1, i}\right) \leqslant \max _{0 \leqslant j \leqslant N(n)-1} \bar{f}\left(a_{n, j, \mathrm{eff}}, a_{n, j}\right) \leqslant \eta_{n}
$$

Moreover, by estimation (5),

$$
\bar{f}\left(\sigma^{u}\left(a_{n+1, i}\right), a_{n+1, i}\right) \leqslant \frac{2}{q_{n, i}}
$$

Therefore, by combining estimates (6), (7), (8), (9), we get:

$$
\begin{gathered}
\bar{f}\left(a\left(T_{n+1}, q_{n+1}, x\right), a_{n+1, i}\right) \leqslant \bar{f}\left(a\left(T_{n+1}, q_{n+1}, x\right), a_{n+1, i, \mathrm{neg}}\right) \\
+\bar{f}\left(a_{n+1, i, \mathrm{neg}}, \sigma^{u}\left[a_{n+1, i, \mathrm{semeff}}\right]\right)+\bar{f}\left(\sigma^{u}\left(a_{n+1, i, \mathrm{semeff}}\right), \sigma^{u}\left(a_{n+1, i}\right)\right) \\
+\bar{f}\left(\sigma^{u}\left(a_{n+1, i}\right), a_{n+1, i}\right) \leqslant 2 \epsilon_{n+1} N_{n+1} \max _{0 \leqslant i \leqslant N(n+1)-1} q_{n, i}+\frac{2 N(n)^{2} q_{n}^{2} q_{n, i}}{q_{n+1}}+\eta_{n}+\max _{0 \leqslant i \leqslant N(n+1)-1} \frac{2}{q_{n, i}}
\end{gathered}
$$

Therefore,

$$
\bar{f}\left(a\left(T_{n+1}, q_{n+1}, x\right), a_{n+1, i}\right) \leqslant \eta_{n+1}
$$

Proof of lemma 3.5. Let $\pi_{a}: a \rightarrow a^{\prime}$ and $\pi_{b}: b \rightarrow b^{\prime}$ two matches. Let $\pi: a b \rightarrow a^{\prime} b^{\prime}$ defined by $\pi_{\mid a}=\pi_{a}$ and $\pi_{\mid b}=\pi_{b} . \pi$ is a match because $\pi_{a}$ and $\pi_{b}$ are matches (it is an order-preserving, injective function). Moreover,

$\operatorname{fit}\left(\pi_{a}\right)+\operatorname{fit}\left(\pi_{b}\right)=\frac{\left|\mathcal{D}\left(\pi_{a}\right)\right|}{\frac{1}{2}\left(|a|+\left|a^{\prime}\right|\right)}+\frac{\left|\mathcal{D}\left(\pi_{b}\right)\right|}{\frac{1}{2}\left(|b|+\left|b^{\prime}\right|\right)}=2 \frac{\left|\mathcal{D}\left(\pi_{a}\right)\right|+\left|\mathcal{D}\left(\pi_{b}\right)\right|}{\frac{1}{2}\left(|a|+\left|a^{\prime}\right|+|b|+\left|b^{\prime}\right|\right)}=2 \operatorname{fit}(\pi)$

Moreover, fit $(\pi) \leqslant 1-\bar{f}\left(a b, a^{\prime} b^{\prime}\right)$. By taking the maximum on possible fits of $\pi_{a}$ and $\pi_{b}$ in the previous equality, we get: $1-\bar{f}\left(a, a^{\prime}\right)+1-\bar{f}\left(b, b^{\prime}\right) \leqslant 2\left(1-\bar{f}\left(a b, a^{\prime} b^{\prime}\right)\right)$. Hence lemma 3.5.

To get nLB, it remains to give a lower bound on $\bar{f}\left(a_{n+1, i}, a_{n+1, j}\right)$, when $i \neq j$. Our method is analogous to [7, p. 34].

Let

$$
u_{n}=\max \left\{\operatorname{fit}(\pi) / \pi: a_{n, i}^{r} \rightarrow a_{n, j}^{s} \text { match, } r, s \in \mathbb{N}, 0 \leqslant i<j \leqslant N(n)-1\right\}
$$

For $i=0, \ldots, N(n+1)-1$, let $r_{n+1, i}=\frac{q_{n+1}}{N(n) q_{n,} q_{n}}$, and for $j=0, \ldots, N(n+1)-1$, $j>i$, let $\lambda_{n, i, j}=q_{n, j} / q_{n, i}$. Note that since $j>i, \lambda_{n, i, j}$ is a positive integer.

We show the slightly stronger lemma: 
Lemma 3.6. We have:

$$
u_{n+1} \leqslant\left(u_{n}+\frac{2}{N(n)}\right)\left(1+\max _{0 \leqslant i<j \leqslant N(n+1)-1} \frac{2 N(n)}{\lambda_{n, i, j}}\right)\left(1+\max _{0 \leqslant i<j \leqslant N(n+1)-1} \frac{2 \lambda_{n, i, j}}{r_{n+1, i}}\right)
$$

Corollary 3.7. If $N(n) \geqslant 2^{n+3} / \epsilon$, and if for any $0 \leqslant i<j \leqslant N(n+1)-1, \lambda_{n, i, j} \geqslant$ $2^{n+5} N(n)$, and $r_{n+1, i} \geqslant 2^{n+5} \lambda_{n, i, j}$, then for any $r, s>0$,

$$
\bar{f}\left(a_{n, i}^{r}, a_{n, j}^{s}\right) \geqslant 1-\epsilon
$$

Proof of lemma 3.6. We denote $\lambda=\lambda_{n, i, j}$. We have:

$$
\begin{aligned}
a_{n+1, i}^{r} & =\left(a_{n, 0}^{r_{n+1, i}} \ldots a_{n, N(n)-1}^{r_{n+1, i}}\right)^{q_{n, i} r} \\
a_{n+1, j}^{s} & =\left(\begin{array}{ll}
a_{n, 0}^{\frac{r_{n+1, i}}{\lambda}} & \ldots a_{n, N(n)-1}^{\frac{r_{n+1, i}}{\lambda}}
\end{array}\right)^{q_{n, i} \lambda \lambda}
\end{aligned}
$$

For $l=0, \ldots, N(n)-1$, let $\alpha_{n, l}=a_{n, l}^{\frac{r_{n+1, i}}{n}}$.

We can write:

$$
a_{n+1, i}^{r}=\left(\alpha_{n, 0}^{\lambda} \ldots \alpha_{n, N(n)-1}^{\lambda}\right)^{q_{n, i} r}=\bar{\alpha}_{n, 0}^{\lambda} \ldots \bar{\alpha}_{n, N(n) q_{n, i} r-1}^{\lambda}
$$

Let

$$
\pi: \mathcal{D}(\pi) \subset a_{n+1, i}^{r} \rightarrow \mathcal{R}(\pi) \subset a_{n+1, j}^{s}
$$

be a match. For $l=0, \ldots, N(n) q_{n, i} r-1$, let $\mathcal{D}(\pi)_{l}=\mathcal{D}(\pi) \cap \bar{\alpha}_{n, l}^{\lambda}$ (i.e. $\mathcal{D}(\pi)_{l}$ is the part of the word $\mathcal{D}(\pi)$ that is included in the subword $\bar{\alpha}_{n, l}^{\lambda}$ of $a_{n+1, i}^{r}$. We have:

$$
\mathcal{D}(\pi)=\mathcal{D}(\pi)_{0} \ldots \mathcal{D}(\pi)_{N(n) q_{n, i} r-1}
$$

Let $\mathcal{R}(\pi)_{l}=\pi\left(\mathcal{D}(\pi)_{l}\right)$. We can write:

$$
a_{n+1, j}^{s}=a_{n+1, j, 0} \ldots a_{n+1, j, N(n) q_{n, i} r-1}
$$

such that $\mathcal{R}(\pi)_{l} \subset a_{n+1, j, l}$, for $l=0, \ldots, N(n) q_{n, i} r-1$.

Let

$$
\pi_{l}: \mathcal{D}(\pi)_{l} \subset \bar{\alpha}_{n, l}^{\lambda} \rightarrow \mathcal{R}(\pi)_{l} \subset a_{n+1, j, l}
$$

be a match, with $\pi_{l}=\pi_{\mid \mathcal{D}(\pi)_{l}}$. We have: $\mathcal{D}\left(\pi_{l}\right)=\mathcal{D}(\pi)_{l}$.

$a_{n+1, j, l}$ is of the form:

$$
a_{n+1, j, l}=\tilde{\alpha}_{l}\left(\alpha_{n, 0} \ldots \alpha_{n, N(n)-1}\right)^{t_{l}} \widehat{\alpha}_{l}
$$

with $t_{l} \geqslant 0$, and such that $\max \left(\left|\tilde{\alpha}_{l}\right|,\left|\hat{\alpha}_{l}\right|\right) \leqslant N(n)\left|\alpha_{n, 0}\right|$.

Moreover, we have $\operatorname{fit}\left(\pi_{l}\right)=\operatorname{fit}\left(\pi_{l}^{-1}\right)$ because $\left|\mathcal{D}\left(\pi_{l}\right)\right|=\left|\mathcal{R}\left(\pi_{l}\right)\right|$. We have:

$$
\pi_{l}^{-1}: \mathcal{R}\left(\pi_{l}\right) \subset \tilde{\alpha}_{l}\left(\alpha_{n, 0} \ldots \alpha_{n, N(n)-1}\right)^{t} \widehat{\alpha}_{l} \rightarrow \mathcal{D}\left(\pi_{l}\right) \subset \bar{\alpha}_{n, l}^{\lambda}
$$

Let

$$
\tilde{\pi}_{l}^{-1}: \mathcal{R}\left(\pi_{l}\right) \subset\left(\alpha_{n, 0} \ldots \alpha_{n, N(n)-1}\right)^{t_{l}+2} \rightarrow \mathcal{D}\left(\pi_{l}\right) \subset \bar{\alpha}_{n, l}^{\lambda}
$$

such that $\tilde{\pi}_{l}^{-1}=\pi_{l}^{-1}$ (we just extend the domain (not the "domain of definition") of the function $\pi_{l}^{-1}$ ). 
Like previously, we can write: $\left(\alpha_{n, 0} \ldots \alpha_{n, N(n)-1}\right)^{t_{l}+2}=\bar{\alpha}_{n, 0} \ldots \bar{\alpha}_{n,\left(t_{l}+2\right) N(n)-1}$.

Moreover, let $\mathcal{R}\left(\pi_{l}\right)_{p}=\mathcal{R}\left(\pi_{l}\right) \cap \bar{\alpha}_{n, p}$, for $p=0, \ldots, N(n)\left(t_{l}+2\right)-1$. We have:

$$
\mathcal{R}\left(\pi_{l}\right)=\mathcal{R}\left(\pi_{l}\right)_{0} \ldots \mathcal{R}\left(\pi_{l}\right)_{N(n)\left(t_{l}+2\right)-1}
$$

Let $\mathcal{D}\left(\pi_{l}\right)_{p}=\pi_{l}^{-1}\left[\mathcal{R}\left(\pi_{l}\right)_{p}\right]$.

We can also write:

$$
\bar{\alpha}_{n, l}^{\lambda}=\alpha_{n, l, 0} \ldots \alpha_{n, l,(t+2) N(n)-1}
$$

for $l=0, \ldots, N(n) q_{n, i} r-1$, with $\alpha_{n, l, p}$ such that $\mathcal{D}\left(\pi_{l}\right)_{p} \subset \alpha_{n, l, p}$. Moreover, since

$$
\left|\mathcal{D}\left(\pi_{l}\right)_{p}\right|=\left|\mathcal{R}\left(\pi_{l}\right)_{p}\right| \leqslant\left|\bar{\alpha}_{n, p}\right|=\left|\alpha_{n, 0}\right|
$$

we can choose $\alpha_{n, l, p}$ such that, if $l=p \bmod N(n),\left|\alpha_{n, l, p}\right| \leqslant\left|\alpha_{n, 0}\right|$.

Let

$$
\pi_{l, p}: \mathcal{R}\left(\pi_{l}\right)_{p} \subset \bar{\alpha}_{n, p} \rightarrow \mathcal{D}\left(\pi_{l}\right)_{p} \subset \alpha_{n, l, p}
$$

$\alpha_{n, l, p}$ is of the form $\alpha_{n, l, p}=\tilde{a} a_{n, l}^{u} \hat{a}$. with $\max (|\tilde{a}|,|\hat{a}|) \leqslant\left|a_{n, l}\right|=q_{n}$. We have: $\mathcal{D}\left(\pi_{l, p}\right)=\mathcal{R}\left(\pi_{l}\right)_{p}$

We have:

$$
\pi_{l, p}: \mathcal{D}\left(\pi_{l, p}\right) \subset \bar{\alpha}_{n, p}=a_{n, p}^{\frac{r_{n+1, i}}{\lambda}} \rightarrow \mathcal{R}\left(\pi_{l, p}\right) \subset \tilde{a} a_{n, l}^{u} \hat{a}
$$

Let

$$
\tilde{\pi}_{l, p}: \mathcal{D}\left(\pi_{l, p}\right) \subset a_{n, p}^{\frac{r_{n+1, i}}{\lambda}} \rightarrow \mathcal{R}\left(\pi_{l, p}\right) \subset a_{n, l}^{u+2}
$$

Let $0 \leqslant p^{\prime}<N(n)$ such that $p^{\prime}=p \bmod N(n)$ and $0 \leqslant l^{\prime}<N(n)$ such that $l^{\prime}=l$ $\bmod N(n)$.

If $p^{\prime}<l^{\prime}$, then fit $\left(\tilde{\pi}_{l, p}\right) \leqslant u_{n}$, by induction hypothesis.

If $p^{\prime}>l^{\prime}$, then we can apply the induction hypothesis to $\left(\tilde{\pi}_{l, p}\right)^{-1}$, and therefore, $\operatorname{fit}\left(\tilde{\pi}_{l, p}\right)=\operatorname{fit}\left(\tilde{\pi}_{l, p}^{-1}\right) \leqslant u_{n}$.

If $p^{\prime}=l^{\prime}$, then fit $\left(\tilde{\pi}_{l, p}\right) \leqslant 1$ (i.e. we cannot say anything).

Now, let us relate fits of $\tilde{\pi}_{l, p}, \pi_{l, p}, \tilde{\pi}_{l}^{-1}, \pi_{l}$ and $\pi$. First, we relate fits of $\pi_{l, p}$ and $\tilde{\pi}_{l, p}$. We have:

$$
\operatorname{fit}\left(\tilde{\pi}_{l, p}\right)=\frac{2\left|\mathcal{D}\left(\pi_{l, p}\right)\right|}{\left(\frac{r_{n+1, i}}{\lambda}+u+2\right)\left|a_{n, l}\right|}
$$

and on the other hand:

$$
\operatorname{fit}\left(\pi_{l, p}\right)=\frac{2\left|\mathcal{D}\left(\pi_{l, p}\right)\right|}{\frac{r_{n+1, i}}{\lambda}\left|a_{n, l}\right|+u\left|a_{n, l}\right|+|\tilde{a}|+|\hat{a}|} \leqslant \frac{2\left|\mathcal{D}\left(\pi_{l, p}\right)\right|}{\left(\frac{r_{n+1, i}}{\lambda}+u\right)\left|a_{n, l}\right|}
$$

Therefore,

$$
\operatorname{fit}\left(\pi_{l, p}\right) \leqslant \frac{\frac{r_{n+1, i}}{\lambda}+u+2}{\frac{r_{n+1, i}}{\lambda}+u} \operatorname{fit}\left(\tilde{\pi}_{l, p}\right) \leqslant\left(1+\frac{2}{\frac{r_{n+1, i}}{\lambda}+u}\right) \operatorname{fit}\left(\tilde{\pi}_{l, p}\right)
$$

Since $u \geqslant 0$, we get:

$$
\operatorname{fit}\left(\pi_{l, p}\right) \leqslant\left(1+\frac{2 \lambda}{r_{n+1, i}}\right) \operatorname{fit}\left(\tilde{\pi}_{l, p}\right)
$$

We relate fits of $\pi_{l, p}$ and $\tilde{\pi}_{l}^{-1}$. We have: 


$$
\operatorname{fit}\left(\pi_{l, p}\right)=\frac{2\left|\mathcal{D}\left(\pi_{l, p}\right)\right|}{\left|\bar{\alpha}_{n, p}\right|+\left|\alpha_{n, l, p}\right|}
$$

Therefore,

$$
\sum_{p=0}^{N(n)\left(t_{l}+2\right)-1}\left|\mathcal{D}\left(\pi_{l, p}\right)\right|=\frac{1}{2} \sum_{p=0}^{N(n)\left(t_{l}+2\right)-1} \operatorname{fit}\left(\pi_{l, p}\right)\left[\left|\bar{\alpha}_{n, p}\right|+\left|\alpha_{n, l, p}\right|\right]
$$

If $p \neq l \bmod N(n)$, then by estimation (10), $\operatorname{fit}\left(\pi_{l, p}\right) \leqslant\left(1+\frac{2 \lambda}{r_{n+1, i}}\right) u_{n}$. If $p=l$ $\bmod N(n)$, we still have fit $\left(\pi_{l, p}\right) \leqslant 1$ (all fits are smaller or equal to one). Therefore, we get:

$$
\begin{aligned}
\sum_{p=0}^{N(n)\left(t_{l}+2\right)-1}\left|\mathcal{D}\left(\pi_{l, p}\right)\right| & \leqslant\left(1+\frac{2 \lambda}{r_{n+1, i}}\right) u_{n} \frac{1}{2} \sum_{p=0, p \neq l}^{N(n)\left(t_{l}+2\right)-1}\left|\bar{\alpha}_{n, p}\right|+\left|\alpha_{n, l, p}\right| \\
+ & \frac{1}{2} \sum_{p=0, p=l}^{N(n)\left(t_{l}+2\right)-1}\left|\bar{\alpha}_{n, p}\right|+\left|\alpha_{n, l, p}\right|
\end{aligned}
$$

On the other hand,

$$
\operatorname{fit}\left(\tilde{\pi}_{l}^{-1}\right)=\frac{2\left|\mathcal{R}\left(\pi_{l}\right)\right|}{\left(t_{l}+2\right) N(n)\left|\alpha_{n, 0}\right|+\left|\bar{\alpha}_{n, l}^{\lambda}\right|}=\frac{2 \sum_{p=0}^{N(n)\left(t_{l}+2\right)-1}\left|\mathcal{D}\left(\pi_{l, p}\right)\right|}{\sum_{p=0}^{N(n)\left(t_{l}+2\right)-1}\left|\bar{\alpha}_{n, p}\right|+\left|\alpha_{n, l, p}\right|}
$$

Therefore,

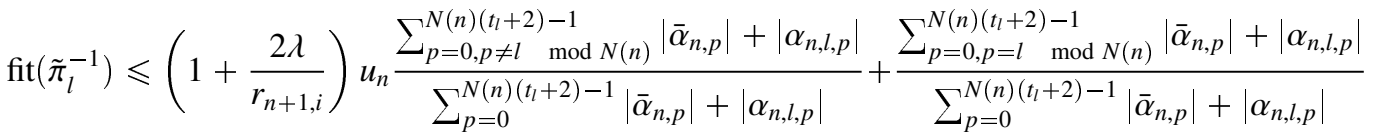

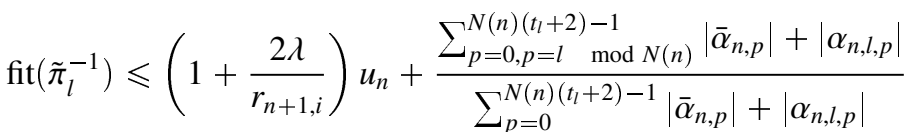

Moreover, $\left|\bar{\alpha}_{n, p}\right|=\left|\alpha_{n, 0}\right|$, and when $p=l \bmod N(n)$, by construction, $\left|\alpha_{n, l, p}\right| \leqslant$ $\left|\alpha_{n, 0}\right|$. We also have:

$$
\sum_{p=0, p=l}^{N(n)\left(t_{l}+2\right)-1} 1 \leqslant t_{l}+2
$$

Therefore,

$$
\operatorname{fit}\left(\tilde{\pi}_{l}^{-1}\right) \leqslant\left(1+\frac{2 \lambda}{r_{n+1, i}}\right) u_{n}+\frac{2\left(t_{l}+2\right)\left|\alpha_{n, 0}\right|}{N(n)\left(t_{l}+2\right)\left|\alpha_{n, 0}\right|+\lambda\left|\alpha_{n, 0}\right|} \leqslant\left(1+\frac{2 \lambda}{r_{n+1, i}}\right) u_{n}+\frac{2}{N(n)}
$$

We relate fits of $\tilde{\pi}_{l}^{-1}$ and $\pi_{l}$. We have: 


$$
\operatorname{fit}\left(\tilde{\pi}_{l}^{-1}\right)=\frac{2\left|\mathcal{R}\left(\pi_{l}\right)\right|}{\left(t_{l}+2\right) N(n)\left|\alpha_{n, 0}\right|+\left|\bar{\alpha}_{n, l}^{\lambda}\right|}=\frac{2\left|\mathcal{R}\left(\pi_{l}\right)\right|}{\left(\left(t_{l}+2\right) N(n)+\lambda\right)\left|\alpha_{n, 0}\right|}
$$

On the other hand, since $a_{n+1, j, l}$ is of the form $a_{n+1, j, l}=\tilde{\alpha}_{l}\left(\alpha_{n, 0} \ldots \alpha_{n, N(n)-1}\right)^{t l} \hat{\alpha}_{l}$, we get:

$$
\operatorname{fit}\left(\pi_{l}\right)=\operatorname{fit}\left(\pi_{l}^{-1}\right)=\frac{2\left|\mathcal{R}\left(\pi_{l}\right)\right|}{(N(n) t+\lambda)\left|\alpha_{n, 0}\right|+\left|\tilde{\alpha}_{l}\right|+\left|\hat{\alpha}_{l}\right|} \leqslant \frac{2\left|\mathcal{R}\left(\pi_{l}\right)\right|}{\left(N(n) t_{l}+\lambda\right)\left|\alpha_{n, 0}\right|}
$$

$\operatorname{fit}\left(\pi_{l}\right) \leqslant \frac{\left(t_{l}+2\right) N(n)+\lambda}{N(n) t_{l}+\lambda} \operatorname{fit}\left(\tilde{\pi}_{l}^{-1}\right) \leqslant\left(1+\frac{2 N(n)}{N(n) t_{l}+\lambda}\right) \operatorname{fit}\left(\tilde{\pi}_{l}^{-1}\right) \leqslant\left(1+\frac{2 N(n)}{\lambda}\right) \operatorname{fit}\left(\tilde{\pi}_{l}^{-1}\right)$

Finally,

$$
\begin{aligned}
& \operatorname{fit}(\pi)=\frac{\sum_{l=0}^{r q_{n, i}-1} 2\left|\mathcal{D}\left(\pi_{l}\right)\right|}{\sum_{l=0}^{r q_{n, i}-1}\left|\alpha_{n, l}^{\lambda}\right|+\left|a_{n+1, j, l}\right|} \leqslant \frac{\sum_{l=0}^{r q_{n, i}-1} \max _{0 \leqslant r q_{n, i}-1}\left(\operatorname{fit}\left(\pi_{l}\right)\right)\left|\alpha_{n, l}^{\lambda}\right|+\left|a_{n+1, j, l}\right|}{\sum_{l=0}^{r q_{n, i}-1}\left|\alpha_{n, l}^{\lambda}\right|+\left|a_{n+1, j, l}\right|} \\
& \leqslant \max _{0 \leqslant l \leqslant r q_{n, i}-1}\left(\operatorname{fit}\left(\pi_{l}\right)\right)
\end{aligned}
$$

By taking the max on all possible fit $(\pi)$, we get:

$$
u_{n+1} \leqslant\left(u_{n}+\frac{2}{N(n)}\right)\left(1+\frac{2 N(n)}{\lambda}\right)\left(1+\frac{2 \lambda}{r_{n+1, i}}\right)
$$

By taking the max on all possible $\lambda$, we get the conclusion.

Proof of corollary 3.7. By induction on $n$, we show:

$$
u_{n} \leqslant \epsilon\left(1-\frac{1}{2^{n}}\right)
$$

If $n=0, u_{0}=0$, so the estimate holds. Suppose the estimate holds at rank $n$. By lemma 3.6,

$$
u_{n+1} \leqslant\left(u_{n}+\frac{2}{N(n)}\right)\left(1+\max _{0 \leqslant i<j \leqslant N(n+1)-1} \frac{2 N(n)}{\lambda_{n, i, j}}\right)\left(1+\max _{0 \leqslant i<j \leqslant N(n+1)-1} \frac{2 \lambda_{n, i, j}}{r_{n+1, i}}\right)
$$

Moreover, for any $i<j$,

$$
\left(1+\frac{2 \lambda_{n, i, j}}{r_{n+1, i}}\right) \leqslant 1+\frac{1}{2^{n+4}}
$$

and

$$
\left(1+\frac{2 N(n)}{\lambda_{n, i, j}}\right) \leqslant 1+\frac{1}{2^{n+4}}
$$

Therefore, 


$$
\left(1+\frac{2 \lambda_{n, i, j}}{r_{n+1, i}}\right)\left(1+\frac{2}{\lambda_{n, i, j}}\right) \leqslant\left(1+\frac{1}{2^{n+4}}\right)^{2}=1+\frac{2}{2^{n+4}}+\frac{1}{\left(2^{n+4}\right)^{2}} \leqslant 1+\frac{1}{2^{n+2}}
$$

Moreover, by induction assumption,

$$
u_{n}+\frac{2}{N(n)} \leqslant \epsilon\left(1-\frac{1}{2^{n}}+\frac{1}{2^{n+2}}\right)
$$

By combining these two estimates, we get:

$$
u_{n+1} \leqslant \epsilon\left(1-\frac{1}{2^{n+1}}\right)
$$

Hence the estimate at step $n+1$.

Proof of proposition 3.2. Let $\epsilon_{n+1}^{\prime}=4 \epsilon_{n+1} N_{n+1}^{2} q_{n, N(n+1)-1}$. Each quasi-permutation constituting $A_{n+1}$ has a Lebesgue density of at most $4 \epsilon_{n+1}$. Moreover, there is less than $N_{n+1}^{2} q_{n, N(n+1)-1}$ quasi-permutations in $A_{n+1}$. Therefore,

$$
\mu\left(\operatorname{turb}\left(A_{n+1}\right)\right) \leqslant \epsilon_{n+1}^{\prime}
$$

By applying estimation (4), we get:

$$
\mu\left(E_{\mathrm{safe}}\right) \geqslant 1-\sum_{n \geqslant 0} \epsilon_{n+1}^{\prime}
$$
then

There exists $f_{\text {turb }}\left(\epsilon, n, N_{n+1}, q_{n, N(n+1)-1}\right)$ such that if $\epsilon_{n+1} \leqslant f_{\text {turb }}\left(\epsilon, n, N_{n+1}, q_{n, N(n+1)-1}\right)$,

$$
\mu\left(E_{\text {safe }}\right) \geqslant 1-\epsilon
$$

Therefore,

$$
\mu\left(E_{\mathrm{safe}} \cap E_{\mathrm{nlb}}\right) \geqslant 1-2 \epsilon
$$

There also exists $f_{\text {dist }}\left(\epsilon, n, N_{n+1}, q_{n, N(n+1)-1}\right)$ such that if $\epsilon_{n+1} \leqslant f_{\text {dist }}\left(\epsilon, n, N_{n+1}, q_{n, N(n+1)-1}\right)$, then $\eta_{n} \leqslant \epsilon$. We take for $\epsilon_{n+1}$ a function of $\epsilon, n, N_{n+1}, q_{n, N(n+1)-1}$ such that

$$
\epsilon_{n+1} \leqslant \min \left(f_{\text {dist }}\left(\epsilon, n, N_{n+1}, q_{n, N(n+1)-1}\right), f_{\text {turb }}\left(\epsilon, n, N_{n+1}, q_{n, N(n+1)-1}\right)\right)
$$

Let $A \in \mathcal{B}$ such that $\mu(A)>2 \epsilon$. Then $\mu\left(A \cap\left(E_{\mathrm{safe}} \cap E_{\mathrm{nlb}}\right)\right)>0$.

Since $N(n) \rightarrow_{n \rightarrow+\infty}+\infty$ then $\mu\left(\max _{0 \leqslant i \leqslant N(n)-1} c_{i}(n)\right) \rightarrow_{n \rightarrow+\infty} 0$. Therefore, for any $n$ sufficiently large, and by applying lemmas 3.3,3.4 and corollary 3.7, there exists $x, y \in A \cap\left(E_{\mathrm{safe}} \cap E_{\mathrm{nlb}}\right)$ and $i \neq j$ such that

$$
\begin{aligned}
& \bar{f}\left(a\left(T, q_{n}, x\right), a_{n, i}\right) \leqslant \eta_{n} \\
& \bar{f}\left(a\left(T, q_{n}, y\right), a_{n, j}\right) \leqslant \eta_{n}
\end{aligned}
$$

Therefore,

$$
\bar{f}\left(a\left(T, q_{n}, x\right), a\left(T, q_{n}, y\right)\right) \geqslant 1-3 \epsilon
$$




\subsection{Ergodicity}

\subsubsection{The case $M=[0,1] \times \mathbb{T}$}

Let

$$
P_{n}=\left\{\left[\frac{i}{N_{n}}, \frac{i+1}{N_{n}}\left[\times\left[\frac{j}{q_{n}}, \frac{j}{q_{n}}\left[, 0 \leqslant i \leqslant N_{n}-1,0 \leqslant j \leqslant q_{n}-1\right\}\right.\right.\right.\right.
$$

Since $P_{n}$ is a partition generating the Lebesgue sigma-algebra, it is sufficient to show that $T$ is ergodic with respect to $\mathcal{B}\left(P_{n}\right)$.

Lemma 3.8. Let $q>0$ that divides $q_{m}$ and $\eta=\left\{\left[i / q,(i+1) / q[, 0 \geqslant i \geqslant q-1\}\right.\right.$. $R \frac{p_{m}}{q_{m}}$ is ergodic with respect to $(\mathcal{B}(\eta), \lambda)$, where $\lambda$ denotes the Lebesgue measure on $\mathbb{T}$, and for any $A, B \in \mathcal{B}(\eta)$,

$$
\frac{1}{q_{m}} \sum_{l=0}^{q_{m}-1} \lambda\left(R_{\frac{l_{m}}{q_{m}}}(A) \cap B\right)=\lambda(A) \lambda(B)
$$

Proof. Let $A \in \mathcal{B}\left(\eta_{n}\right), \lambda(A)>0$ that is $R_{\frac{p_{m}}{q_{m}}}$-invariant. Then there is $0 \leqslant i_{0} \leqslant$ $q_{n}-1$ such that $\left[i_{0} / q_{n},\left(i_{0}+1\right) / q_{n}\left[\subset A\right.\right.$. Therefore, $\mathbb{T}=\cup_{0 \leqslant i \leqslant q_{m}-1} R_{\frac{i p m}{q_{m}}}\left(\left[i_{0} / q_{n},\left(i_{0}+\right.\right.\right.$ 1) $/ q_{n}[) \subset A$, and $R \frac{p_{m}}{q_{m}}$ is $\mathcal{B}\left(\eta_{n}\right)$-ergodic. By $q_{m}$-periodicity and the ergodic theorem, for any integer $L>0$,

$$
\frac{1}{q_{m}} \sum_{i=0}^{q_{m}-1} \lambda\left(R_{\frac{i p_{m}}{q_{m}}}(A) \cap B\right)=\frac{1}{L q_{m}} \sum_{i=0}^{L q_{m}-1} \lambda\left(R_{\frac{i p_{m}}{q_{m}}}(A) \cap B\right) \rightarrow_{L \rightarrow+\infty} \lambda(A) \lambda(B)
$$

Now, we define the finite algebra $\mathcal{B}\left(\zeta_{n}\right)$ that contains the elements of $A_{n+1}\left(P_{n}\right)$, modulo small turbulences (see figure 7). Let

$C=\left\{l=\left(l_{i^{\prime}, j}\right), 0 \leqslant i^{\prime} \leqslant N_{n+1}-1,0 \leqslant j \leqslant \frac{q_{n, i}}{q_{n, 0}}, 0 \leqslant i \leqslant N(n+1)-1, i=i^{\prime} \quad \bmod N(n+1)\right\}$

For $l \in C$, let

$$
C(l)=\bigcup_{i=0}^{N(n+1)-1} \bigcup_{0 \leqslant i^{\prime} \leqslant N_{n+1}, i^{\prime}=i}\left[\frac{i^{\prime}}{N_{n+1}}, \frac{i^{\prime}+1}{N_{n+1}}\left[\times \cup_{j=0}^{\frac{q_{n, i}}{q_{n, 0}}-1}\left[\frac{l_{i, j}}{N_{n} q_{n, i}}, \frac{l_{i, j}+1}{N_{n} q_{n, i}}[\right.\right.\right.
$$

Lemma 3.9. Let $\zeta_{n}=\{C(l), l \in C\}$ ( $\zeta_{n}$ recovers $[0,1] \times \mathbb{T}$, but it is not a partition). For any $m>n, S \frac{p_{m}}{q_{m}}$ is ergodic with respect to $\mathcal{B}\left(\zeta_{n}\right)$, and for any $A, B \in \mathcal{B}\left(\zeta_{n}\right)$,

$$
\frac{1}{q_{m}} \sum_{l=0}^{q_{m}-1} \mu\left(S_{\frac{l p m}{q_{m}}}(A) \cap B\right)=\mu(A) \mu(B)
$$

Proof. For $i^{\prime}=0, \ldots, N_{n+1}-1$, let

$$
P_{i^{\prime}}=\left\{\left[\frac{i^{\prime}}{N_{n+1}}, \frac{i^{\prime}+1}{N_{n+1}}\left[\times\left[\frac{j}{N_{n} q_{n, i}}, \frac{j+1}{N_{n} q_{n, i}}\left[, 0 \leqslant j \leqslant N_{n} q_{n, i}-1\right\}\right.\right.\right.\right.
$$




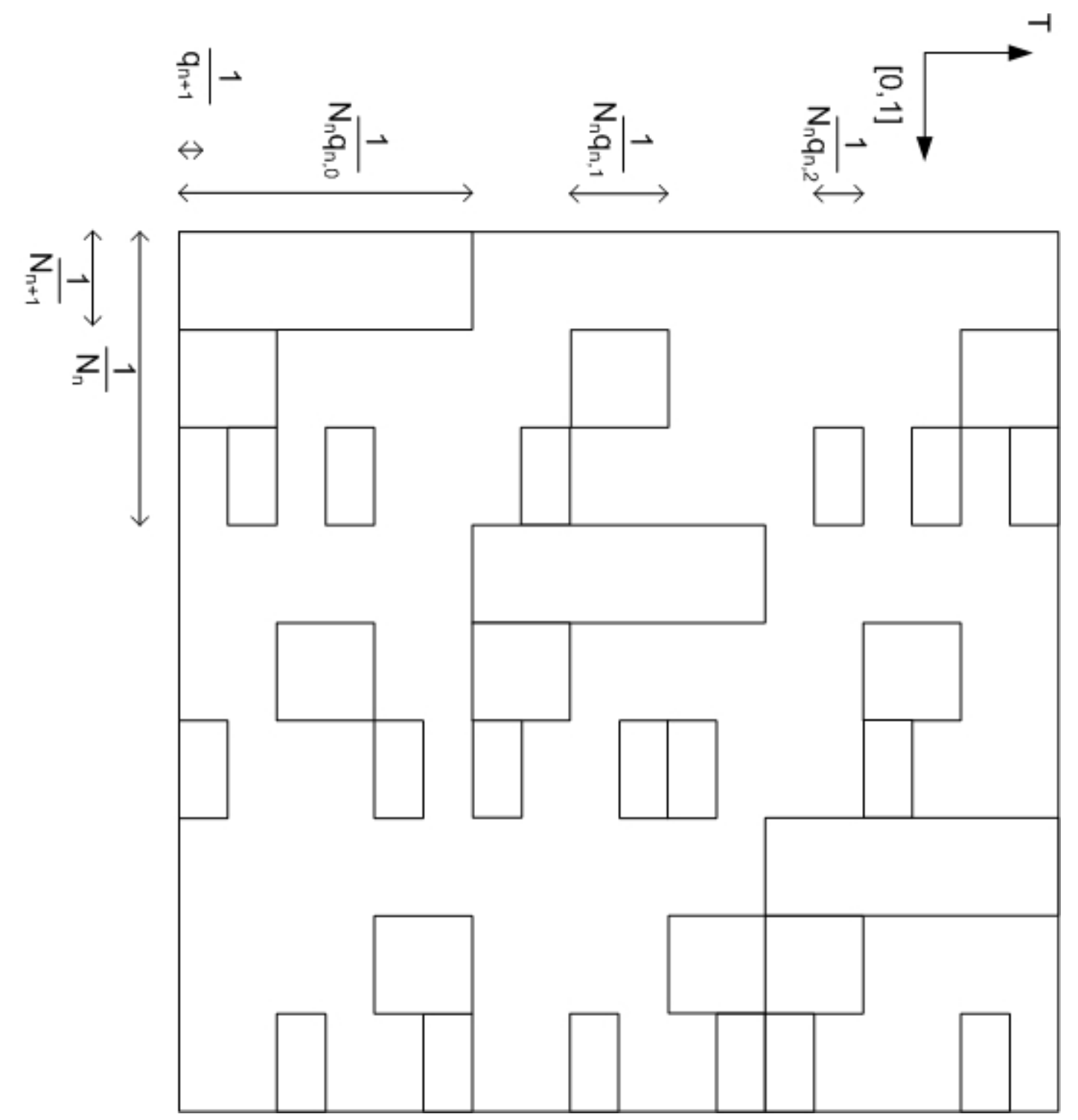

Figure 7: An element of $\zeta_{n}$ with $N_{n}=3, q_{n, 0}=1, q_{n, 1}=3 q_{n, 0}, q_{n, 2}=2 q_{n, 1} . S_{\frac{p m}{q m}}$ is ergodic with respect to $\mathcal{B}\left(\zeta_{n}\right)$. 
$\left(S \frac{p_{m}}{q m} \mid \mathcal{B}\left(P_{i^{\prime}}\right), \mu_{\mid\left[\frac{i^{\prime}}{N_{n+1}}, \frac{i^{\prime}+1}{N_{n+1}}[\times \mathbb{T}\right.}\right)$ is metrically isomorphic to $\left(R_{\frac{p_{m}}{q m}}, \eta_{N_{n} q_{n, i}}, \lambda\right)$ via the map

$$
\pi:\left[\frac{i^{\prime}}{N_{n+1}}, \frac{i^{\prime}+1}{N_{n+1}}\left[\times\left[\frac{j}{N_{n} q_{n, i}}, \frac{j+1}{N_{n} q_{n, i}}\left[\mapsto \left[\frac{j}{N_{n} q_{n, i}}, \frac{j+1}{N_{n} q_{n, i}}[\right.\right.\right.\right.\right.
$$

In particular,

$$
\lambda\left(\pi \left(A \cap\left[\frac{i^{\prime}}{N_{n+1}}, \frac{i^{\prime}+1}{N_{n+1}}[\times \mathbb{T})\right)=\mu(A)\right.\right.
$$

Moreover, for any $A \in \mathcal{B}\left(\zeta_{n}\right),\left[i^{\prime} / N_{n+1},\left(i^{\prime}+1\right) / N_{n+1}\left[\times \mathbb{T} \cap A \in \mathcal{B}\left(P_{i^{\prime}}\right)\right.\right.$. Therefore, we have:

$$
\begin{aligned}
& \frac{1}{q_{m}} \sum_{l=0}^{q_{m}-1} \mu\left(S_{\frac{l p_{m}}{q_{m}}}(A) \cap B\right) \\
& =\frac{1}{q_{m}} \sum_{l=0}^{q_{m}-1} \sum_{i^{\prime}=0}^{N_{n+1}-1} \mu\left(S _ { \frac { l p _ { m } } { q _ { m } } } \left(A \cap \left[\frac{i^{\prime}}{N_{n+1}}, \frac{i^{\prime}+1}{N_{n+1}}[\times \mathbb{T}) \cap\left(B \cap\left[\frac{i^{\prime}}{N_{n+1}}, \frac{i^{\prime}+1}{N_{n+1}}[\times \mathbb{T})\right)\right.\right.\right.\right. \\
& =\frac{1}{q_{m}} \sum_{l=0}^{q_{m}-1} \sum_{i^{\prime}=0}^{N_{n+1}-1} N_{n+1} \mu_{\mid\left[\frac{i^{\prime}}{N_{n+1}}, \frac{i^{\prime}+1}{N_{n+1}}[\times \mathbb{T}\right.}\left(S _ { \frac { p _ { m } } { q _ { m } } } \left(A \cap \left[\frac{i^{\prime}}{N_{n+1}}, \frac{i^{\prime}+1}{N_{n+1}}[\times \mathbb{T}) \cap\left(B \cap\left[\frac{i^{\prime}}{N_{n+1}}, \frac{i^{\prime}+1}{N_{n+1}}[\times \mathbb{T})\right)\right.\right.\right.\right. \\
& =\frac{1}{q_{m}} \sum_{l=0}^{q_{m}-1} \sum_{i^{\prime}=0}^{N_{n+1}-1} N_{n+1} \mu_{\mid\left[\frac{i^{\prime}}{N_{n+1}}, \frac{i^{\prime}+1}{N_{n+1}}[\times \mathbb{T}\right.}\left(S _ { \frac { l _ { p m } } { q _ { m } } } \left(A \cap \left[\frac{i^{\prime}}{N_{n+1}}, \frac{i^{\prime}+1}{N_{n+1}}[\times \mathbb{T}) \cap\left(B \cap\left[\frac{i^{\prime}}{N_{n+1}}, \frac{i^{\prime}+1}{N_{n+1}}[\times \mathbb{T})\right)\right.\right.\right.\right. \\
& =\frac{1}{q_{m}} \sum_{l=0}^{q_{m}-1} \sum_{i^{\prime}=0}^{N_{n+1}-1} N_{n+1} \lambda\left(R _ { \frac { l p m } { q _ { m } } } \left(\pi \left(A \cap [ \frac { i ^ { \prime } } { N _ { n + 1 } } , \frac { i ^ { \prime } + 1 } { N _ { n + 1 } } [ \times \mathbb { T } ) ) \cap \pi \left(\left(B \cap\left[\frac{i^{\prime}}{N_{n+1}}, \frac{i^{\prime}+1}{N_{n+1}}[\times \mathbb{T})\right)\right)\right.\right.\right.\right. \\
& =\frac{1}{q_{m}} \sum_{l=0}^{q_{m}-1} \sum_{i^{\prime}=0}^{N_{n+1}-1} N_{n+1} \lambda\left(R _ { \frac { l _ { m } } { q _ { m } } } \left(\pi \left(A \cap [ \frac { i ^ { \prime } } { N _ { n + 1 } } , \frac { i ^ { \prime } + 1 } { N _ { n + 1 } } [ \times \mathbb { T } ) ) \cap \pi \left(\left(B \cap\left[\frac{i^{\prime}}{N_{n+1}}, \frac{i^{\prime}+1}{N_{n+1}}[\times \mathbb{T})\right)\right)\right.\right.\right.\right. \\
& =\frac{1}{N_{n+1}} N_{n+1} \lambda\left(\pi \left(A \cap [ \frac { i ^ { \prime } } { N _ { n + 1 } } , \frac { i ^ { \prime } + 1 } { N _ { n + 1 } } [ \times \mathbb { T } ) ) \lambda \left(\pi \left(B \cap\left[\frac{i^{\prime}}{N_{n+1}}, \frac{i^{\prime}+1}{N_{n+1}}[\times \mathbb{T})\right)=\mu(A) \mu(B)\right.\right.\right.\right.
\end{aligned}
$$

Lemma 3.10. For any $A, B \in P_{n}$,

$$
\frac{1}{q_{m}} \sum_{l=0}^{q_{m}-1} \mu\left(T_{m}^{l}(A) \cap B\right) \rightarrow_{m \rightarrow+\infty} \mu(A) \mu(B)
$$


Proof. By construction, there exists $A^{\prime}, B^{\prime} \in \mathcal{B}\left(\zeta_{m}\right)$ such that

$$
\begin{gathered}
\mu\left(A^{\prime} \bar{f} B_{m}(A)\right) \leqslant \mu\left(\operatorname{turb}\left(B_{m}\right)\right)=\epsilon_{m}^{\prime \prime} \\
\mu\left(B^{\prime} \bar{f} B_{m}(B)\right) \leqslant \epsilon_{m}^{\prime \prime}
\end{gathered}
$$

Therefore,

$$
\begin{gathered}
\frac{1}{q_{m}} \sum_{l=0}^{q_{m}-1} \mu\left(T_{m}^{l}(A) \cap B\right)=\frac{1}{q_{m}} \sum_{l=0}^{q_{m}-1} \mu\left(S_{l \frac{p_{m}}{q_{m}}} B_{m}(A) \cap B_{m}(B)\right) \\
\leqslant \frac{1}{q_{m}} \sum_{l=0}^{q_{m}-1} \mu\left(S_{l \frac{p_{m}}{q_{m}}} B_{m}\left(A^{\prime}\right) \cap B_{m}\left(B^{\prime}\right)\right)+\mu\left(A^{\prime} \bar{f} B_{m}(A)\right)+\mu\left(A^{\prime} \bar{f} B_{m}(A)\right)+\mu\left(B^{\prime} \bar{f} B_{m}(B)\right)
\end{gathered}
$$

By lemma 3.9, we get

$$
\begin{gathered}
\frac{1}{q_{m}} \sum_{l=0}^{q_{m}-1} \mu\left(T_{m}^{l}(A) \cap B\right) \leqslant \mu\left(A^{\prime}\right) \mu\left(B^{\prime}\right)+2 \epsilon_{m}^{\prime \prime} \\
\leqslant\left[\mu\left(B_{m}(A)\right)+\mu\left(A^{\prime} \bar{f} B_{m}(A)\right)\right]\left[\mu\left(B_{m}(B)\right)+\mu\left(B^{\prime} \bar{f} B_{m}(B)\right)\right]+2 \epsilon_{m}^{\prime \prime} \\
\leqslant \mu(A) \mu(B)+5 \epsilon_{m}^{\prime \prime}
\end{gathered}
$$

Therefore,

$$
\limsup _{m \rightarrow+\infty} \frac{1}{q_{m}} \sum_{l=0}^{q_{m}-1} \mu\left(T_{m}^{l}(A) \cap B\right) \leqslant \mu(A) \mu(B)
$$

Likewise,

$$
\liminf _{m \rightarrow+\infty} \frac{1}{q_{m}} \sum_{l=0}^{q_{m}-1} \mu\left(T_{m}^{l}(A) \cap B\right) \geqslant \mu(A) \mu(B)
$$

Lemma 3.11. For any $A, B \in \mathcal{B}\left(P_{n}\right)$,

$$
\frac{1}{q_{m}} \sum_{l=0}^{q_{m}-1} \mu\left(T^{l}(A) \cap B\right) \rightarrow_{m \rightarrow+\infty} \mu(A) \mu(B)
$$

Proof.

$$
\begin{aligned}
\frac{1}{q_{m}} \sum_{l=0}^{q_{m}-1} \mu\left(T^{l}(A)\right. & \cap B) \leqslant \frac{1}{q_{m}} \sum_{l=0}^{q_{m}-1} \mu\left(T_{m}^{l}(A) \cap B\right)+\max _{0 \leqslant l \leqslant q_{m}-1} d_{0}\left(T^{l}, T_{m}^{l}\right) \\
& =\mu(A) \mu(B)+\max _{0 \leqslant l \leqslant q_{m}-1} d_{0}\left(T^{l}, T_{m}^{l}\right)
\end{aligned}
$$

By estimate (2), we get 


$$
\frac{1}{q_{m}} \sum_{l=0}^{q_{m}-1} \mu\left(T^{l}(A) \cap B\right) \leqslant \mu(A) \mu(B)+\frac{\epsilon}{q_{m} N(0) 2^{m+1}}
$$

Therefore,

$$
\limsup _{m \rightarrow+\infty} \frac{1}{q_{m}} \sum_{l=0}^{q_{m}-1} \mu\left(T^{l}(A) \cap B\right) \leqslant \mu(A) \mu(B)
$$

Likewise,

$$
\liminf _{m \rightarrow+\infty} \frac{1}{q_{m}} \sum_{l=0}^{q_{m}-1} \mu\left(T^{l}(A) \cap B\right) \geqslant \mu(A) \mu(B)
$$

Lemma 3.12. $T$ is ergodic with respect to $\mathcal{B}\left(P_{n}\right)$.

Proof. Let $A \in \mathcal{B}\left(P_{n}\right) T$-invariant modulo zero. By taking $B=A$ in lemma 3.11, we get $\mu(A)=(\mu(A))^{2}$, and so $\mu(A)=0$ or 1 .

3.3.2 Construction in the case $M=[0,1]^{d-1} \times \mathbb{T}, d \geqslant 3$

The construction in the case $M=[0,1]^{d-1} \times \mathbb{T}, d \geqslant 3$ is the same as in the case of the annulus, except that we "fold" other dimensions to obtain ergodicity, in the same way as in $[6,4,3]$. However, the proof of ergodicity needs to be different from those works.

For $n \geqslant 1$, let

$$
\begin{gathered}
P_{n}=\left\{\left[\frac{i_{1}}{N_{n}}, \frac{i_{1}+1}{N_{n}}\left[\times\left[\frac{i_{2}}{q_{n-1}}, \frac{i_{2}+1}{q_{n-1}}\left[\times \ldots \times\left[\frac{i_{d}}{q_{n-1}}, \frac{i_{d}+1}{q_{n-1}}[,\right.\right.\right.\right.\right.\right. \\
\left.0 \leqslant i_{1} \leqslant N_{n}-1,0 \leqslant i_{j} \leqslant q_{n-1}-1,2 \leqslant j \leqslant d\right\}
\end{gathered}
$$

Since the diameter of elements of $P_{n}$ tends to zero as $n \rightarrow+\infty$, then as in the case of the annulus, it is sufficient to show that $T$ is ergodic with respect to $\mathcal{B}\left(P_{n}\right)$, in order to get ergodicity with respect to the Lebesgue algebra.

Let also, for $i=0, \ldots, N_{n}-1$,

$$
\zeta_{n, i}=\left\{\left[\frac{i}{N_{n}}, \frac{i+1}{N_{n}}\left[\times[0,1]^{d-2} \times\left[\frac{i_{d}}{q_{n-1}^{d-1}}, \frac{i_{d}+1}{q_{n-1}^{d-1}}\left[, 0 \leqslant i_{j} \leqslant q_{n-1}^{d-1}-1,2 \leqslant j \leqslant d\right\}\right.\right.\right.\right.
$$

In our construction of the sequence $q_{n}$, we can assume that $q_{n-1}^{d-1}$ divides $q_{n}$. Therefore, by lemma 3.8, for any $i=0, \ldots, N_{n}-1$ fixed, $S \frac{p_{n}}{q_{n}} \mid \mathcal{B}\left(\zeta_{n, i}\right)$ is ergodic.

We denote by $\tilde{A}_{n+1}=\left(\tilde{A}_{n+1,1}, \tilde{A}_{n+1,2}\right)$ the map $A_{n+1}$ of the annulus case, and $A_{n+1}^{1}\left(x_{1}, \ldots, x_{d}\right)=\left(\tilde{A}_{n+1,1}\left(x_{1}, x_{d}\right), x_{2}, \ldots, x_{d-1}, \tilde{A}_{n+1,2}\left(x_{1}, x_{d}\right)\right)$. We denote by $A_{n+1}^{2}$ the application that "folds" other dimensions, i.e. that essentially transforms $P_{n}$ into $\cup_{i=0}^{N_{n}-1} \zeta_{n, i}$ (except on turbulences). We define $A_{n+1}^{2}$ below. Moreover, in the definition of $P_{n}$, we took larger elements, because after their compression by $A_{n+1}^{2}$, they need to be sufficiently wide so that $A_{n+1}^{1}$ can give ergodicity (we use that $q_{n-1}^{d-1} \leqslant q_{n} \leqslant N_{n} q_{n, i}$ ). We let $A_{n+1}=A_{n+1}^{1} A_{n+1}^{2}$. Now, we define $A_{n+1}^{2}$.

We recall the definition of a "quasi-rotation" by $\pi / 2$ [6]: 
Proposition 3.13. For any $n \geqslant 1$, there is a smooth measure preserving map $\phi_{n}$ : $[0,1]^{2} \rightarrow[0,1]^{2}$ (called "quasi-rotation") such that $\phi_{n}=R_{\pi / 2}$ on $\left[\frac{1}{2^{n}}, 1-\frac{1}{2^{n}}\right]^{2}$ and $\phi_{n}=$ Id on $[0,1]^{2}-\left[\frac{1}{2^{n+1}}, 1-\frac{1}{2^{n+1}}\right]^{2}$.

Let $p \geqslant 2$ and

$$
\begin{aligned}
C_{p}:[0,1] \times\left[0, \frac{1}{p}\right] & \rightarrow[0,1] \times[0,1] \\
(x, y) & \mapsto(x, p y)
\end{aligned}
$$

Let $\phi_{n, p}=C_{p}^{-1} \phi_{n} C_{p}$. The map $\phi_{n, p}$ is measure preserving. By the Faa-di-Bruno formula, there exists a fixed function $R_{2}(j)$ such that

$$
\left\|\phi_{n, p}\right\|_{j} \leqslant p^{R_{2}(j)}\left\|\phi_{n}\right\|_{j}
$$

For $i=1, \ldots, d-1$, let $\phi_{n, q_{n-1}}^{i}\left(x_{1}, \ldots, x_{d}\right)=\left(x_{1}, . ., x_{i-1}, \phi_{n, q_{n-1}}\left(x_{i}, x_{i+1}\right), x_{i+2}, \ldots, x_{d}\right)$, extended by $1 / q_{n-1}$-equivariance along the $x_{i+1}$ coordinate. We let

$$
A_{n+1}^{2}\left(x_{1}, \ldots, x_{d}\right)=\phi_{n, q_{n-1}}^{d-1} \ldots \phi_{n, q_{n-1}}^{1}\left(x_{1}, \ldots, x_{d}\right)
$$

Modulo turbulence zones, $A_{n+1}^{2}$ essentially transforms an element of $P_{n}$ into an element of $\zeta_{n, i}$ for some $i$, i.e. into a parallelepipede of height $1 / q_{n-1}^{d-1}$ along the $x_{d}$ coordinate, of width $1 / N_{n}$ along the coordinate $x_{1}$, and of width 1 on all other dimensions. Then, we use the ergodicity of $S \frac{p_{n}}{q_{n}} \mid \mathcal{B}\left(\zeta_{n, i}\right)$ as we used the ergodicity of $S \frac{p_{n}}{q_{n}} \mid \mathcal{B}\left(\zeta_{n}\right)$ in the case of dimension 2. Then, we can proceed with $A_{n+1}^{1}$ (because $q_{n-1}^{d-1}$ divides $N_{n} q_{n, i}$ for any $i$ ) as in dimension 2 to get ergodicity.

\subsubsection{The general case}

We apply the proposition, found in $[1,6,4,3]$ :

Proposition 3.14 ([6]). Let $M$ be a d-dimensional smooth compact connected manifold, with a free modulo zero circle action $\hat{S}$, preserving a smooth volume $\mu$. Let $S_{t}$ denote the circle action on $[0,1]^{d-1} \times \mathbb{T}$. There exists a continuous surjective map $\Gamma:[0,1]^{d-1} \times \mathbb{T} \rightarrow M$ such that:

1. the restriction of $\Gamma$ to $] 0,1\left[{ }^{d-1} \times \mathbb{T}\right.$ is a smooth diffeomorphic embedding.

2. $\mu\left(\Gamma\left(\partial\left([0,1]^{d-1} \times \mathbb{T}\right)\right)\right)=0$

3. $B \subset \Gamma\left(\partial\left([0,1]^{d-1} \times \mathbb{T}\right)\right)$

4. $\Gamma_{*}(L e b)=\mu$

5. $\widehat{S} \Gamma=\Gamma S$

Let $\widehat{T}_{n}: M \rightarrow M$ defined by $\widehat{T}_{n}(x)=\Gamma B_{n}^{-1} S_{\frac{p_{n}}{q_{n}}} B_{n} \Gamma^{-1}(x)$ if $x \in \Gamma(] 0,1\left[{ }^{d-1} \times \mathbb{T}\right)$ and $\widehat{T}_{n}(x)=\widehat{S}_{\frac{p_{n}}{q_{n}}}(x)$ otherwise.

We proceed as in [4]: we take $q_{n+1}$ large enough so that the possible divergence of $\Gamma$ on the border of $] 0,1\left[^{d-1} \times \mathbb{T}\right.$ does not affect the convergence of $\widehat{T}_{n}$ towards a smooth, $\mathrm{nLB}$ and ergodic diffeomorphism. 


\section{Generalization to an uncountable family of pairwise non-Kakutani equivalent diffeomorphisms}

In order to obtain an uncountable family of pairwise non-Kakutani equivalent diffeomorphisms, we adapt the construction of Rudolph, Ornstein and Weiss [12], which generalizes the construction of Feldman. Let $u \in\{0,1\}^{\mathbb{N}}$ be a sequence of 0 and 1 . We construct a family $T_{u}$ of diffeomorphisms in the following way: if $u_{n+1}=0$, then we define the diffeomorphism $A_{n+1}$, appearing in the successive conjugacies, as previously, i.e. such that, up to small perturbations, the $q_{n+1}$-trajectories are of the form:

$$
a_{n+1, i}=\left(a_{n, 0}^{\frac{q_{n+1}}{N(n) q_{n, i} q_{n}}} a_{n, 1}^{\frac{q_{n+1}}{N(n) q_{n, i} q_{n}}} \ldots a_{n, N(n)-1}^{\frac{q_{n+1}}{N(n) q_{n, i} q_{n}}}\right)^{q_{n, i}}
$$

On the other hand, if $u_{n+1}=1$, we define the diffeomorphism $A_{n+1}$ such that, up to small perturbations, the $q_{n+1}$-trajectories are of the form:

$$
a_{n+1, i}=\left(a_{n, N(n)-1}^{\frac{q_{n+1}}{N(n) q_{n} q_{n}}} \ldots a_{n, 1}^{\frac{q_{n+1}}{N(n) q_{n, i} q_{n}}} a_{n, 0}^{\frac{q_{n+1}}{N(n) q_{n, i} q_{n}}}\right)^{q_{n, i}}
$$

Thus, the constructions of $T_{u}$ and $T$ are analogous, we do not write the explicit definition of $A_{n+1}$ for $T_{u}$. We have:

Theorem 4.1. If $u, v \in\{0,1\}^{\mathbb{N}}$ such that $u_{n} \neq v_{n}$ infinitely many times. Then $T_{u}$ and $T_{v}$ are not Kakutani-equivalent.

To show theorem 4.1, we follow and adapt the proof of Ornstein, Rudolph, and Weiss [12]. The proof is based on two ideas: the first idea, as in the nLB case, is that the two words $a a a a b b b b$ and $a b a b a b a b$ are far from each other in the $\bar{f}$-distance. The second idea is that the two words $a b c a b c a b c$ and bcabcabca are also far from each other in the $\bar{f}$-distance (see figure 15 ). We can adapt their proof to the smooth case for two reasons: first, turbulences from $A_{n+1}$ are "grouped" in vanishingly small places, and consequently, they rarely affect $q_{n+1}$-trajectories, and second, turbulences from $B_{n}$ are periodic in the $q_{n+1}$-trajectories. However, the detailed proof is a little technical. We need to introduce some additional definitions.

\subsection{Definitions}

\subsubsection{The distances $\bar{f}$ and $\bar{d}$}

The definitions recalled here are mainly taken from [12, p.8]. First, we can generalize the distance $\bar{f}$ to a semi-distance on infinite words. Let

$$
w=\ldots a_{-1} a_{0} a_{1} \ldots \quad w^{\prime}=\ldots a_{-1}^{\prime} a_{0}^{\prime} a_{1}^{\prime} \ldots
$$
by:

be infinite words, i.e. $w, w^{\prime} \in I^{\mathbb{Z}}$. Let $w_{n}, w_{n}^{\prime} \in I^{2 n+1}$ be the truncated words defined

$$
w_{n}=a_{-n} \ldots a_{-1} a_{0} a_{1} \ldots a_{n} \quad w^{\prime}=a_{-n}^{\prime} \ldots a_{-1}^{\prime} a_{0}^{\prime} a_{1}^{\prime} \ldots a_{n}^{\prime}
$$

Let $\bar{f}\left(w, w^{\prime}\right)=\lim \sup _{n \rightarrow+\infty} \bar{f}\left(w_{n}, w_{n}^{\prime}\right)$.

Likewise, we can define the Hamming distance $\bar{d}\left(w_{n}, w_{n}^{\prime}\right)=\#\left\{i / a_{i} \neq a_{i}^{\prime}\right\}$ and $\bar{d}\left(w, w^{\prime}\right)=\lim \sup _{n \rightarrow+\infty} \bar{d}\left(w_{n}, w_{n}^{\prime}\right)$. 
Now, we define the $\bar{f}$ and $\bar{d}$ distances between two ergodic transformations $T$ and $T^{\prime}$ coded with the partitions $P$ and $P^{\prime}$ respectively ( $T$ and $T^{\prime}$ are not necessarily defined on the same space, and possibly $P \neq P^{\prime}$ ).

Let $v, v^{\prime}$ be two measures on $I^{\mathbb{Z}}$ and let $v_{n}, v_{n}^{\prime}$ be two measures on $I^{n}$ defined by $v, v^{\prime}$ via the projection $I^{\mathbb{Z}} \rightarrow I^{n}$ onto the coordinates $(1, \ldots, n)$. Let:

$$
\bar{f}\left(v_{n}, v_{n}^{\prime}\right)=\inf _{\lambda} \int_{I^{n} \times I^{n}} \bar{f}\left(w_{n}, w_{n}^{\prime}\right) d \lambda
$$
let:

Where the inf is taken on measures $\lambda$ on $I^{n} \times I^{n}$ whose marginals are $v_{n}$ and $v_{n}^{\prime}$. We

$$
\bar{f}\left(v, v^{\prime}\right)=\inf \left\{\epsilon>0 / \bar{f}\left(v_{n}, v_{n}^{\prime}\right) \leqslant \epsilon \text { for an infinity of } n\right\}
$$

Let $T: M \rightarrow M$ be an ergodic transformation, and $P$ be a measurable partition indexed by $I$ that is generating, i.e. $\mathcal{B}=\mathcal{B}\left(\bigvee_{i=-\infty}^{+\infty} T^{i}(P)\right)$. Then $(T, M, \mu)$ is metrically isomorphic to $\left(\sigma, I^{\mathbb{Z}}, v\right)$, where $\sigma$ is the shift on $I^{\mathbb{Z}}$ and preserves $v$, and we have: $\mu\left(c_{i}\right)=v\left(\pi_{0}^{-1}(i)\right)$, where $\pi_{0}: I^{\mathbb{Z}} \rightarrow I$ is the projection on the coordinate 0 . Let $v, v^{\prime}$ associated with $(T, P),\left(T^{\prime}, P^{\prime}\right)$ respectively. We define:

$$
\begin{aligned}
& \bar{f}\left((T, P),\left(T^{\prime}, P^{\prime}\right)\right)=\bar{f}\left(v, v^{\prime}\right) \\
& \bar{d}\left((T, P),\left(T^{\prime}, P^{\prime}\right)\right)=\bar{d}\left(v, v^{\prime}\right)
\end{aligned}
$$

We also use the proposition $[12, \mathrm{p} .8]$ :

Proposition 4.2. If $\bar{f}\left(v, v^{\prime}\right)=\epsilon$, then there are generic points $x, x^{\prime}$ for $\mu, \mu^{\prime}$ respectively such that

$$
\bar{f}\left(x, x^{\prime}\right) \leqslant \epsilon
$$

Let $P=\left\{c_{0}, \ldots, c_{N(0)-1}\right\}$ and $P^{\prime}=\left\{c_{0}^{\prime}, \ldots, c_{N(0)-1}^{\prime}\right\}$ two measurable partitions of the same size. Their distance is defined by:

$$
d\left(P, P^{\prime}\right)=\min _{\theta \in \varsigma_{N(0)}} \sum_{i=0}^{N(0)-1} \mu\left(c_{i} \Delta c_{\theta(i)}^{\prime}\right)
$$

where $\Im_{N(0)}$ denotes the set of permutations of $\{0, \ldots, N(0)-1\}$, and $\Delta$ denotes the symmetric difference.

Let $\mu(A)>0$ and $r_{A, T}: A \rightarrow \mathbb{N}^{*}$, defined by $r_{A, T}(x)=\min \left\{k \geqslant 1, T^{k}(x) \in A\right\}$, be the first return map of $T$ in $A$. By the Poincaré recurrence theorem, $r_{A, T}$ is finite almost everywhere, and by ergodicity (see [12, p.1]),

$$
\int_{A} r_{A, T}(x) d \mu(x)=1
$$

We denote by $\mu_{A}=\mu(.) / \mu(A)$ the measure induced by $\mu$ on $A$, and $\mathcal{B}_{A}$ the sigmaalgebra induced by $\mathcal{B}$. For almost every $x \in A$, let

$$
T_{A}(x)=T^{r_{A, T}(x)}(x)
$$

$T_{A}$ is the transformation induced by $T$ on $A$. $T_{A}$ is a measure preserving transformation of $\left(A, \mathcal{B}_{A}, \mu_{A}\right)$. 
Let also $S$ be an ergodic and measure preserving transformation of $(M, \mathcal{B}, \mu) . T$ and $S$ are Kakutani-equivalent (Russians called it monotone equivalent [8]) if there exists $A, B \in \mathcal{B}$ such that $\mu(A)>0, \mu(B)>0$, and such that $T_{A}$ and $S_{B}$ are metrically isomorphic.

\subsubsection{The tower construction}

We introduce the tower construction (see figure 8). Let $g: M \rightarrow \mathbb{N}^{*}$ integrable. Let

$$
\begin{gathered}
M^{g}=\left\{(x, i) \in M \times \mathbb{N}^{*}, 1 \leqslant i \leqslant g(x)\right\} \\
T_{v}^{g}: M^{g} \rightarrow M^{g} \\
T_{v}^{g}(x, i)= \begin{cases}(x, i+1) & \text { if } i+1 \leqslant g(x) \\
\left(T_{v}(x), 1\right) & \text { if } i+1>g(x)\end{cases}
\end{gathered}
$$

If $T_{v}$ is ergodique, then $T_{v}^{g}$ is also ergodic. Let $P=\left\{c_{0}, c_{1}, \ldots, c_{N(0)-1}\right\}$ be a measurable partition of $M$. Let $H=\left\{(x, i) \in M^{g} / i \geqslant 2\right\}$, and $P^{g}=\left\{c_{0}, c_{1}, \ldots, c_{N(0)-1}, H\right\}$ be the corresponding measurable partition of $M^{g}$. If $\left(T_{v}^{g}\right)^{j}(x, i) \in H$, the corresponding letter in the trajectory is denoted $h$. The $\left(T_{v}^{g}, P^{g}\right)$-trajectory is obtained from the $\left(T_{v}^{g}, P^{g}\right)$-trajectory by adding letters $h$.

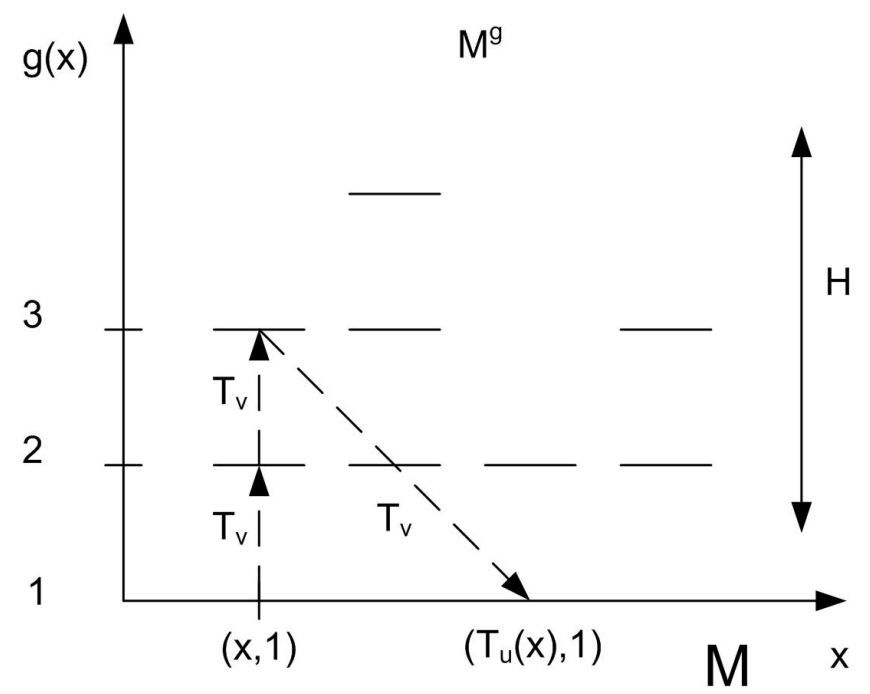

Figure 8: The tower construction $\left(M^{g}, T_{v}^{g}, P^{g}\right)$

We will also need the lemma:

Lemma 4.3. For any $\gamma>0$, there exists an integer $N$, a set $E_{N} \subset M^{g}$ such that $\mu\left(E_{N}\right) \geqslant 1-\gamma$ and such that if $(x, i) \in E_{N}$ and $n \geqslant N$, then

$$
\left|\frac{1}{n} \#\left\{j \in\{1, \ldots, n\} /\left(T_{v}^{g}\right)^{j}(x, i) \in H\right\}-\mu(H)\right| \leqslant \gamma
$$


Proof. For any integer $n$, let

$$
E_{n}=\left\{(x, i) \in M^{g} / \text { if } p \geqslant n \text { then }\left|\frac{1}{p} \#\left\{j \in\{1, \ldots, p\} /\left(T_{v}^{g}\right)^{j}(x, i) \in H\right\}-\mu(H)\right| \leqslant \gamma\right.
$$

The sequence of $E_{n}$ is increasing for the inclusion, and by the ergodic theorem, $\mu\left(\bigcup_{n \geqslant 0} E_{n}\right)=1$. Therefore, $\mu\left(M^{g}-E_{n}\right) \rightarrow_{n \rightarrow+\infty} 0$. Let $N$ such that $\mu\left(M^{g}-E_{N}\right) \leqslant \gamma$. We have $\mu\left(E_{N}\right) \geqslant 1-\gamma$.

In subsection 4.2, we show:

Proposition 4.4. For any $g: M \rightarrow \mathbb{N}^{*}$ integrable, such that $g \neq 1$ ( 1 is the constant function equal to 1$)$, and any sequences $u, v($ even if $u=v), T_{u}$ and $T_{v}^{g}$ are not isomorphic.

Corollary 4.5. If $\mu(A) \neq \mu(B)$, then $\left(T_{u}\right)_{A}$ and $\left(T_{v}\right)_{B}$ are not isomorphic.

In subsection 4.3, we show:

Proposition 4.6. If $\mu(A)=\mu(B)$, and $u_{n} \neq v_{n}$ infinitely many times, then $\left(T_{u}\right)_{A}$ and $\left(T_{v}\right)_{B}$ are not isomorphic (i.e. $T_{u}$ and $T_{v}$ are not evenly equivalent).

By combining corollary 4.5 and proposition 4.6, we obtain theorem 4.1.

Proof of corollary 4.5. We show how proposition 4.4 implies corollary 4.5. By absurd, we suppose that $\left(T_{u}\right)_{A}$ is isomorphic to $\left(T_{v}\right)_{B}$. We can suppose $\mu(A)<\mu(B)$. Let $\Phi:\left(A, \mu_{A}\right) \rightarrow\left(B, \mu_{B}\right)$ a metric isomorphism such that $\Phi\left(T_{u}\right)_{A}=\left(T_{v}\right)_{B} \Phi$.

For any $g: M \rightarrow \mathbb{N}^{*}$ integrable, $\left(\left(T_{u}\right)_{A}\right)^{g}$ is isomorphic to $\left(\left(T_{v}\right)_{B}\right)^{g \circ \Phi^{-1}}$ via the isomoprhism $\tilde{\Phi}: M^{g} \bigcirc$ defined by $\tilde{\Phi}((x, i))=(\Phi(x), i)$. We have:

$$
\int_{B} r_{B, T_{v}}\left(x^{\prime}\right) d \mu_{B}\left(x^{\prime}\right)=\frac{1}{\mu(B)}
$$

On the other hand, since $\Phi:\left(A, \mu_{A}\right) \rightarrow\left(B, \mu_{B}\right)$ is an isomorphism, then

$$
\int_{B} r_{A, T_{u}}\left(\Phi^{-1}\left(x^{\prime}\right)\right) d \mu_{B}\left(x^{\prime}\right)=\int_{A} r_{A, T_{u}}(x) d \mu_{A}(x)=\frac{1}{\mu(A)}>\frac{1}{\mu(B)}
$$

Moreover, by relation (7) in [12, p.2], $T_{u}$ is isomorphic to $\left(T_{u}\right)_{A}^{r_{A, T_{u}}}$, which is isomorphic to $\left(T_{v}\right)_{B}^{r_{A, T_{u}} \circ \Phi^{-1}}$. Likewise, $T_{v}$ is isomorphic to $\left(T_{v}\right)_{B}^{r_{B, T_{v}}}$. Therefore, by lemma 1.3 of [12, p.3], there exists $g: M \rightarrow \mathbb{N}^{*}$ integrable, $g \neq 1$, such that $T_{v}^{g}$ is isomorphic to $T_{u}$. This contradicts proposition 4.4 .

\section{2 $T_{u}$ and $T_{v}^{g}$ are not isomorphic}

By absurd, we suppose there is a metric isomorphism $\Phi:\left(T_{u}, P\right) \rightarrow\left(T_{v}^{g}, P^{g}\right)$. Then for any $\tau>0$, there exists $K(\tau)>0, P(\tau) \subset \bigvee_{i=-K(\tau)}^{K(\tau)} T_{u}^{i}(P)$ such that $|P(\tau)|=\left|P^{g}\right|$ and $d\left(\Phi^{-1}\left(P^{g}\right), P(\tau)\right) \leqslant \tau$, where $d$ denotes the distance between partitions. We can put an equivalence relation on $\bigvee_{i=-K(\tau)}^{K(\tau)} T_{u}^{i}(P)$ : two elements $Q_{1}, Q_{2}$ of this partition 
are equivalent if there exists $Q_{3} \in P(\tau)$ that contains both $Q_{1}$ and $Q_{2}$. We denote $\bar{Q}_{1}$ this equivalent class.

For $x \in E_{n l b}$, we consider the $\left(T_{u}, P\right)$-trajectory of $x$ :

$$
\ldots a_{-1} a_{0} a_{1} \ldots
$$

where $T_{u}^{i}(x) \in c_{a_{i}}$. From this $\left(T_{u}, P\right)$-trajectory, we can derive a $\left(T_{u}, \bigvee_{i=-K(\tau)}^{K(\tau)} T_{u}^{i}(P)\right)$ trajectory:

$$
\begin{gathered}
\left(a_{-K(\tau)-1} a_{-K(\tau)} \ldots a_{-1} a_{0} \ldots a_{K(\tau)-1}\right)\left(a_{-K(\tau)} a_{-K(\tau)+1} \ldots a_{0} a_{1} \ldots a_{K(\tau)}\right) \\
\left(a_{-K(\tau)+1} a_{-K(\tau)+2} \ldots a_{1} a_{2} \ldots a_{K(\tau)+1}\right)
\end{gathered}
$$

where $\left(a_{-K(\tau)+i} a_{-K(\tau)+i+1} \ldots a_{i} a_{i+1} \ldots a_{K(\tau)+i}\right)$ is such that:

$$
x \in T_{u}^{-(i-K(\tau))}\left(c_{a_{i-K(\tau)}}\right) \cap \ldots \cap T_{u}^{-(i+K(\tau))}\left(c_{a_{i+K(\tau)}}\right)
$$

By taking the equivalent classes, we can derive a $\left(T_{u}, P(\tau)\right)$-trajectory (an overline denotes the equivalent class):

$$
\begin{gathered}
\left(\overline{a_{-K(\tau)-1} a_{-K(\tau)} \ldots a_{-1} a_{0} \ldots a_{K(\tau)-1}}\right)\left(\overline{a_{-K(\tau)} a_{-K(\tau)+1} \ldots a_{0} a_{1} \ldots a_{K(\tau)}}\right) \\
\left(\overline{a_{-K(\tau)+1} a_{-K(\tau)+2} \ldots a_{1} a_{2} \ldots a_{K(\tau)+1}}\right)
\end{gathered}
$$

where $\left(\overline{a_{-K(\tau)+i} a_{-K(\tau)+i+1} \ldots a_{i} a_{i+1} \ldots a_{K(\tau)+i}}\right)$ is such that:

$$
x \in \overline{T_{u}^{-(i-K(\tau))}\left(c_{a_{i-K(\tau)}}\right) \cap \ldots \cap T_{u}^{-(i+K(\tau))}\left(c_{a_{i+K(\tau)}}\right)}
$$

We also consider the $\left(T_{u}, \Phi^{-1}\left(P^{g}\right)\right)$-trajectory of $x$, which is identified with the $\left(T_{v}^{g}, P^{g}\right)$-trajectory of $\Phi(x)$. It corresponds to a $\left(T_{v}, P\right)$-trajectory, in which we insert letters $h$. Thus, this trajectory is of the form:

$$
\ldots b_{-1} h h b_{0} h b_{1} b_{2} h h h \ldots
$$

Since, by absurd, we assumed

$$
d\left(\Phi^{-1}\left(P^{g}\right), P(\tau)\right) \leqslant \tau
$$

then by the ergodic theorem,

$$
\bar{d}\left(a\left(T_{u}, \Phi^{-1}\left(P^{g}\right), x\right), a\left(T_{u}, P(\tau), x\right)\right)=\bar{d}\left(a\left(T_{v}^{g}, P^{g}, \Phi(x)\right), a\left(T_{u}, P(\tau), x\right)\right) \leqslant \tau
$$

We let $\tau=\frac{1}{64} \frac{1-4 \epsilon}{\int g}\left(1-\frac{1}{\int g}\right)$, and to get a contradiction, we show:

Proposition 4.7. For any $x \in E_{n l b}$,

$$
\bar{d}\left(a\left(T_{v}^{g}, P^{g}, \Phi(x)\right), a\left(T_{u}, P(\tau), x\right)\right) \geqslant \frac{1}{32} \frac{1-4 \epsilon}{\int g}\left(1-\frac{1}{\int g}\right)
$$

Proof. The proof of proposition 4.7 has two steps. First (lemma 4.8), when segments of the $\left(T_{u}, P\right)$-trajectory of $x$, and segments of the $\left(T_{v}^{g}, P^{g}\right)$-trajectory of $\Phi(x)$, have different types, we show that the $\bar{d}$-distance between their $\left(T_{u}, P(\tau)\right)$ and $\left(T_{v}^{g}, P^{g}\right)$ trajectories is larger than a fixed bound. 
Indeed, in this case, repetitions of the words $a_{n, i, \text { eff }}$ in the $\left(T_{u}, P\right)$-trajectory, and repetitions of the words $\tilde{a}_{n, i, \text { eff }}$ in the $\left(T_{v}^{g}, P^{g}\right)$-trajectory, have different periodicities (moreover, the periodicity of repetitions of $\check{a}_{n, i, \text { eff }}$ in the $\left(T_{u}, P(\tau)\right)$-trajectory is the same as the periodicity of repetitions of $a_{n, i \text {,eff }}$ in the $\left(T_{u}, P\right)$-trajectory, because $K(\tau)<$ $\left.q_{n} / 2\right)$. Turbulences remain packed in rare locations, and do not sensibly affect $\bar{d}$.

Second (lemma 4.9), we show that segments of the $\left(T_{u}, P\right)$ and $\left(T_{v}^{g}, P^{g}\right)$-trajectories are not very often of the same type, because the $T_{v}^{g}$-trajectory is an expansion of the $T_{v}$-trajectory by $g$. On average, the trajectory is expanded by a factor $\int g$ because of the ergodic theorem, and as a consequence, only $\frac{1}{\int g}$ of segments are of the same type, which reduces the $\bar{d}$-distance by a factor $1-\frac{1}{\int g}$. Combining lemmas 4.8 and 4.9 gives proposition 4.7 .

First, we explain how we decompose the $\left(T_{u}, P\right)$ and $\left(T_{v}^{g}, P^{g}\right)$-trajectories in overlaps of segments of the same type. By lemma 3.3, the $q_{n+2}$-trajectories of $x \in E_{n l b}$ with respect to $\left(T_{u}, P\right)$ and $\left(T_{n+2, u}, P\right)$ are the same $\left(T_{n+2, u}\right.$ is the periodic approximation of $T_{u}$ at step $\left.n+2\right)$. If we neglect turb $\left(A_{n+2}\right)$, they are of the form:

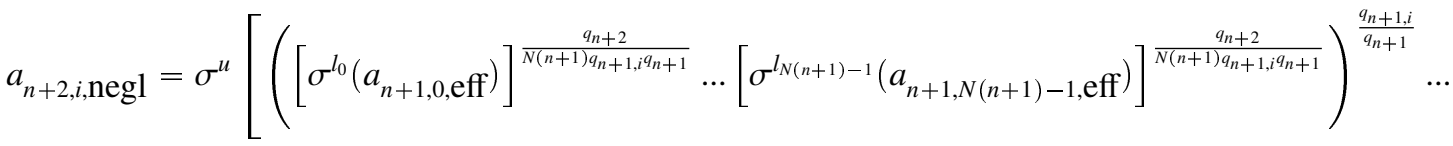

$$
\begin{aligned}
& \left.\left(\left[\sigma^{q_{n+2}-1+l_{0}}\left(a_{n+1,0, \mathrm{eff}}\right)\right]^{\frac{q_{n+2}}{N(n+1) q_{n+1, i} q_{n+1}}} \ldots\left[\sigma^{q_{n+1}-1+l_{N(n+1)-1}}\left(a_{n+1, N(n+1)-1, \mathrm{eff}}\right)\right]^{\frac{q_{n+2}}{N(n+1) q_{n+1, i} q_{n+1}}}\right)^{\frac{q_{n+1, i}}{q_{n+1}}}\right]
\end{aligned}
$$

where $\sigma$ is a circular permutation (see proof of lemma 3.4 page 16).

What is important is that at $x$ and $i$ fixed, the $a_{n+1, i, \text { eff }}$ are identical. Therefore, the $\left(T_{u}, P(\tau)\right) q_{n+2}$-trajectory of $x$ will have the same form most of the time (i.e. repeated

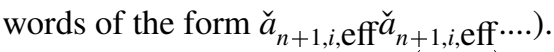

We take into account turb $\left(A_{n+2}\right)$. Given the localization of $\operatorname{turb}\left(A_{n+2}\right)$, the $\left(T_{u}, P\right)$ $q_{n+2}$-trajectory of $x$ meets a new turbulence zone of $A_{n+2}$ at most every $q_{n+2} /\left(N_{n+2} q_{n+1, N(n+2)}\right)$ iterations (see figure 4). This $q_{n+2}$-trajectory is of the form, where $t$ denotes letters in $\operatorname{turb}\left(A_{n+2}\right)$ (or boundary effects due to the cyclic permutation $\sigma$, which have total lengths $\left.q_{n+1}\right)$ :

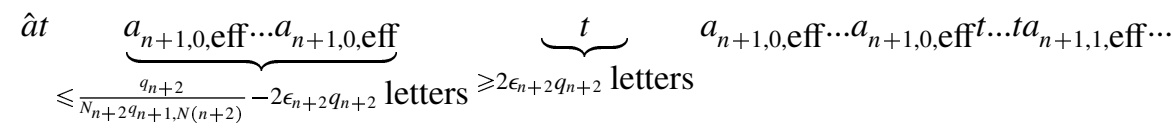

with $|\hat{a}| \leqslant q_{n+2} / q_{n+1,0}$. The $\left(T_{v}^{g}, P^{g}\right)$-trajectory of $\Phi(x)$ is of the form:

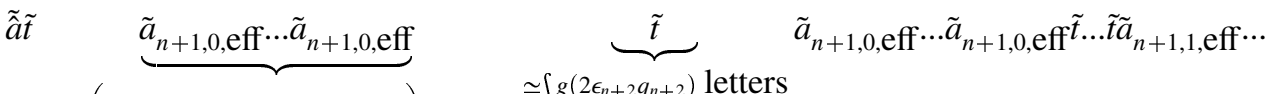

$$
\begin{aligned}
& \simeq \int g\left(\frac{q_{n+2}}{N_{n+2} q_{n+1, N(n+2)}}-2 \epsilon_{n+2} q_{n+2}\right) \text { letters } \simeq \int g\left(2 \epsilon_{n+2} q_{n+2}\right) \text { letters }
\end{aligned}
$$

Tilded words are like untilded words, except that we added letters $h$ in them.

We decompose $\mathbb{Z}$, the set of indices of trajectories, into

$$
\ldots \tilde{t} G_{-1} G_{0} G_{1} \ldots G_{\frac{q_{n+2}}{N_{n+2} q_{n+1, N(n+2)}}} \ldots t G_{2} \ldots \tilde{t} \ldots
$$


where $G_{i}=a_{n+1, l, \text { eff }} \cap \tilde{a}_{n+1, l^{\prime} \text {, eff }}$, i.e. $G_{i}$ is the intersection of the sets of indices of an element $a_{n+1, l, \text { eff in }}\left(T_{u}, P\right)$-trajectory, and of the set of indices of an element $\tilde{a}_{n+1, l^{\prime}, \text { eff }}$ in the $\left(T_{v}^{g}, P^{g}\right)$-trajectory. $G_{i}$ is an overlap of a $n+1$-block in the $\left(T_{u}, P\right)$ trajectory and a $n+1$-block in the $\left(T_{v}^{g}, P^{g}\right)$-trajectory (see figure 9 ).

In the decomposition of $\mathbb{Z}$ in different $G_{i}$, we put aside turbulences, because in general $2 \epsilon_{n+2} q_{n+2}=|t|>>\left|G_{i}\right| \simeq q_{n+1}$. One turbulence is much larger than any individual $G_{i}$ (when the turbulence comes from $A_{n+2}$, not from the effect of the cyclic permutation).

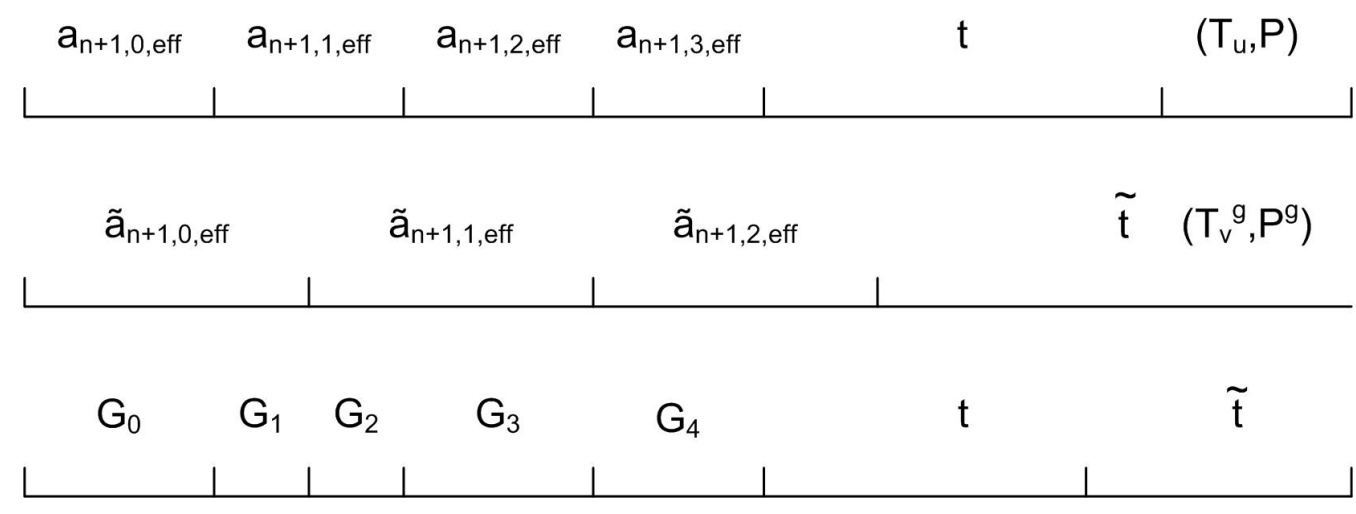

Figure 9: The decomposition of $\mathbb{Z}$ into $G_{i}$ and turbulences $t$ and $\tilde{t}$.

To show proposition 4.7, we combine the lemmas:

Lemma 4.8. Let $\frac{1}{2 \int g}>\epsilon^{\prime}>0$. In $G_{i}=a_{n+1, l, \text { eff }} \cap \tilde{a}_{n+1, l^{\prime}, \text { eff, }}$ if the $n+1$-types $l_{1}$ of $a_{n+1, l, \text { eff }}$ and $l_{1}^{\prime}$ of $\tilde{a}_{n+1, l^{\prime}, \text { eff }}$ are different, and if $\left|G_{i}\right| \geqslant \epsilon^{\prime} q_{n+1}$, then for $n$ sufficiently large,

$$
\bar{d}\left(G_{i}\left(T_{v}^{g}, P^{g}\right), G_{i}\left(T_{u}, P(\tau)\right)\right) \geqslant\left(1-\epsilon^{\prime}\right) \frac{1-4 \epsilon}{32 \int g}
$$

Lemma 4.9. For any $\left(\int g-1\right) / 2 \geqslant \epsilon^{\prime}>0$, and almost every $x \in E_{n l b}$, for $n$ sufficiently large, the density of the set of indices $\left\{G_{i} / l_{1}=l_{1}^{\prime}\right\}$ is less than $1 / \int g+\epsilon^{\prime}$.

Proof of proposition 4.7. We show that for any $\epsilon^{\prime}>0$ such that $\epsilon^{\prime}<\frac{\int g-1}{2}, \epsilon^{\prime}<\frac{1}{2 \int g}$, for any $x \in E_{n l b}$,

$$
\bar{d}\left(a\left(T_{v}^{g}, P^{g}, \Phi(x)\right), a\left(T_{u}, P(\tau), x\right)\right) \geqslant\left(1-3 \epsilon^{\prime}\right)\left(1-\epsilon^{\prime}\right) \frac{1}{32} \frac{1-4 \epsilon}{\int g}\left(1-\frac{1}{\int g}-\epsilon^{\prime}\right)
$$

For almost every $x \in E_{n l b}$, in the $\left(T_{u}, P\right)$-trajectory of $x$, the density of turbulences $t$ from $A_{n+2}$ (and from cyclic permutation $\sigma$ ) is less than $2 \epsilon_{n+2} N_{n+2} q_{n+1, N(n+2)}$.

Moreover, for almost every $x \in M$, in the $\left(T_{v}^{g}, P^{g}\right)$-trajectory of $\Phi(x)$, by lemma 4.3 , for $n$ sufficiently large, the density of turbulences $\tilde{t}$ from $A_{n+2}$ is less than $\epsilon^{\prime}$. Therefore, for $n$ sufficiently large, the density of indices $G_{i}$ in $\mathbb{Z}$ is more than $1-2 \epsilon^{\prime}$

Moreover, in each $a_{n+1, l, \text { eff }}$, there is at most one $G_{i}$ such that $\left|G_{i}\right|<\epsilon^{\prime} q_{n+1}$. Therefore, for $n$ sufficiently large, the total density of $G_{i}$ such that $\left|G_{i}\right| \geqslant \epsilon^{\prime} q_{n+1}$ is greater than $1-3 \epsilon^{\prime}$. Therefore, by combining lemmas 4.8 and 4.9 , we get (12). 
Proof of lemma 4.8. First, we suppose $l_{1}>l_{1}^{\prime}$, i.e. $q_{n, l_{1}}>q_{n, l_{1}^{\prime}}\left(\right.$ and $\left.r_{n, l_{1}}<r_{n, l_{1}^{\prime}}\right)$.

We denote $G_{i}\left(T_{u}, P\right)$ the $\left(T_{u}, P\right)$-trajectory of $x$ on the set of indices $G_{i}$. We can write

$$
G_{i}\left(T_{u}, P\right)=\widehat{a} G_{i, 1}\left(T_{u}, P\right) \ldots G_{i, w}\left(T_{u}, P\right) \widehat{a}^{\prime}
$$

where:

$$
G_{i, l}\left(T_{u}, P\right)=a_{n, 1, \mathrm{eff}} \ldots a_{n, 1, \mathrm{eff}} t \ldots . t a_{n, N(n), \mathrm{eff}} \cdots a_{n, N(n), \mathrm{eff}}{ }^{t}
$$

where $|\hat{a}| \leqslant q_{n+1} / q_{n, l_{1}}$ and $\left|\hat{a}^{\prime}\right| \leqslant q_{n+1} / q_{n, l_{1}}$, i.e.

$$
G_{i}\left(T_{u}, P\right)=\hat{a} \underbrace{a_{n, 1, \mathrm{eff}} \ldots t \ldots a_{n, N(n), \mathrm{eff}}}_{G_{i, 1}\left(T_{u}, P\right)} \underbrace{a_{n, 1, \mathrm{eff}} \ldots t \ldots a_{n, N(n), \mathrm{eff}}}_{G_{i, 2}\left(T_{u}, P\right)} \ldots
$$

The $G_{i, l}\left(T_{u}, P\right)$ are complete cycles of $n$-types. $G_{i, l}\left(T_{u}, P\right)$ include turbulences from $A_{n+1}$, because their density in $G_{i, l}\left(T_{u}, P\right)$ is vanishingly small (although $|t|>>a_{n, i, \text { eff }}$ for each $i$ ).

Since at $i$ fixed, all the $a_{n, i, \text { eff }}$ are identical, and since $K(\tau)<q_{n}$, then $G_{i, l}\left(T_{u}, P(\tau)\right)$ is of the form:

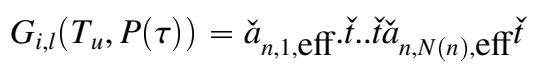

Moreover, $|\tilde{t}| \leqslant|t|(2 K(\tau)+1)$. Therefore, for any integers $j, k$, and for $n$ sufficiently large,

$$
\bar{f}\left(G_{i, j}\left(T_{u}, P(\tau)\right), G_{i, k}\left(T_{u}, P(\tau)\right)\right) \leqslant 2(2 K(\tau)+1) 2 \epsilon_{n+1} N_{n+1} q_{n, N(n+1)} \leqslant \frac{1}{2^{n+2}}
$$

On the other hand, we can write (we do not neglect boundary effects similar to $\hat{a}$ and $\hat{a}^{\prime}$ here):

$$
G_{i}\left(T_{v}^{g}, P^{g}\right)=\bar{G}_{i, 1}\left(T_{v}^{g}, P^{g}\right) \ldots \bar{G}_{i, \bar{w}}\left(T_{v}^{g}, P^{g}\right)
$$

such that:

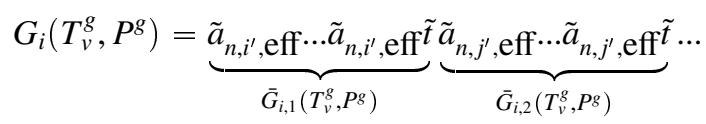

Modulo turbulences, $\bar{G}_{i, j}\left(T_{v}^{g}, P^{g}\right)$ is a segment of the same $n$-type.

If $1<j<\bar{w}$, then $\left|\bar{G}_{i, j}\right| \geqslant \frac{q_{n+1}}{N(n) q_{n, l_{1}^{\prime}}}$. Since $\left|G_{i, j}\right| \leqslant \frac{q_{n+1}}{q_{n, l_{1}}}$, then $\bar{G}_{i, j}$ contains at least $\frac{q_{n, l_{1}}}{\left(N(n) q_{n, l_{1}^{\prime}}\right.}-1 \geqslant 2^{n}$ sets of indices $G_{i, j}$. We can write:

$$
\bar{G}_{i, j}=\hat{a} G_{i, j_{1}} \ldots G_{i, j_{u}} \hat{a}^{\prime}
$$

with $j_{u}-j_{1} \geqslant 2^{n}$. Therefore, $\hat{a}$ and $\hat{a}^{\prime}$ occupy a density of less than $1 / 2^{n}$ of $\bar{G}_{i, j}$. Therefore,

$$
\bar{f}\left(\bar{G}_{i, j}\left(T_{u}, P(\tau)\right), \bar{G}_{i, k}\left(T_{u}, P(\tau)\right)\right) \leqslant\left(1+1 / 2^{n}\right) \frac{1}{2^{n+2}}
$$

For $j=1$ or $\bar{w}$, if $\left|\bar{G}_{i, j}\right| \geqslant 2^{n} \frac{q_{n+1}}{q_{n, 1}}$, the same reasoning applies.

If $\left|\bar{G}_{i, j}\right|<2^{n} \frac{q_{n+1}}{q_{n, l_{1}}}$, then we do not do this estimate. However, we become able to neglect this segment: at most two $\bar{G}_{i, j}$ in $G_{i}$ are short like that. Therefore, in the worst 
case, since $\left|G_{i}\right| \geqslant q_{n+1} \epsilon^{\prime}$, then these boundary effects only take a fraction $2^{n} /\left(q_{n, l_{1}} \epsilon^{\prime}\right)$ of $G_{i}$, and we can write:

$$
G_{i}=\hat{G} \bar{G}_{i, 2} \ldots \bar{G}_{i, \bar{w}-1} \hat{G}^{\prime}
$$

with $|\hat{G}|$ and $\left|\hat{G}^{\prime}\right| \leqslant 2^{n} q_{n+1} / q_{n, l_{1}}$

We want a lower bound on $\bar{f}\left(\bar{G}_{i, j}\left(T_{v}^{g}, P^{g}\right), \bar{G}_{i, k}\left(T_{v}^{g}, P^{g}\right)\right)$, when they are of different $n$-types.

For $i_{1} \neq j_{1}\left(0 \leqslant i_{1} \leqslant N(n)-1\right.$ and $0 \leqslant j_{1} \leqslant N(n)-1$ are $n$-types $)$, and for $n$ sufficiently large, by combining corollary 3.7 and lemma 3.4 , we get:

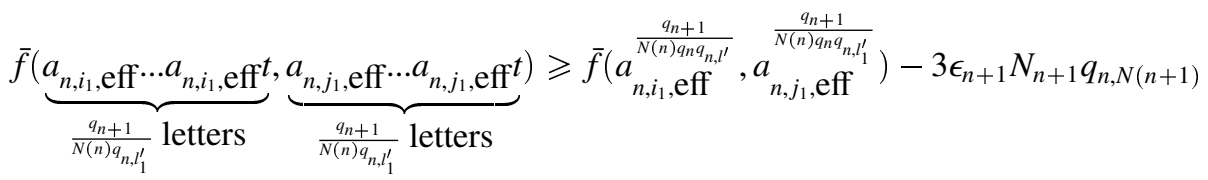

$$
\begin{aligned}
& \geqslant 1-3 \epsilon-\epsilon=1-4 \epsilon
\end{aligned}
$$

To get a lower bound on $\bar{f}\left(\bar{G}_{i, j}\left(T_{v}^{g}, P^{g}\right), \bar{G}_{i, k}\left(T_{v}^{g}, P^{g}\right)\right)$, we need the following lemma, which is straightforward:

Lemma 4.10. Let two words $A, A^{\prime}$ on the alphabet $I$, and we obtain $\bar{A}$ and $\bar{A}^{\prime}$ by inserting at most $(\beta-1)|A|$ letters $h$ in $A$, and $(\beta-1)\left|A^{\prime}\right|$ letters $h$ in $A^{\prime}$, where $h$ is a letter not in the alphabet $I$. We have:

$$
\bar{f}\left(\bar{A}, \bar{A}^{\prime}\right) \geqslant \frac{\bar{f}\left(A, A^{\prime}\right)}{\beta}
$$

By lemma 4.3, we have, for $n$ sufficiently large, except on a set of density less than $\epsilon^{\prime}$ :

$$
\left|\frac{\left|\bar{G}_{i, j}\right|-\frac{q_{n+1}}{N(n) q_{n, l_{1}}}}{\left|\bar{G}_{i, j}\right|}-\frac{\int g-1}{\int g}\right| \leqslant \frac{1}{2 \int g}
$$

Therefore, except on a set of density less than $\epsilon^{\prime}$,

$$
\frac{\left|\bar{G}_{i, j}\right|}{\frac{q_{n+1}}{N(n) q_{n, l_{1}}}} \leqslant 2 \int g
$$

By lemma 4.10 and estimate (15), we get, except on a set of density less than $\epsilon^{\prime}$, for $\bar{G}_{i, j}\left(T_{v}^{g}, P^{g}\right)$ and $\bar{G}_{i, k}\left(T_{v}^{g}, P^{g}\right)$ of different $n$-types:

$$
\bar{f}\left(\bar{G}_{i, j}\left(T_{v}^{g}, P^{g}\right), \bar{G}_{i, k}\left(T_{v}^{g}, P^{g}\right)\right) \geqslant \frac{1-4 \epsilon}{2 \int g}
$$

We denote by $F_{0}$ the set of $\bar{G}_{i, j}$ that do not satisfy (16). For $n$ sufficiently large, the set of indices $\left\{\bar{G}_{i, j} / \bar{G}_{i, j} \in F_{0}\right\}$ has a density of less than $\epsilon^{\prime}$. The number $w^{\prime}$ of segments $\bar{G}_{i, j}$ satisfying (16) satisfies, for $n$ sufficiently large:

$$
w^{\prime} \geqslant \epsilon^{\prime}\left(1-\epsilon^{\prime}\right) q_{n+1} \frac{N(n) q_{n, l_{1}}}{q_{n+1} 2 \int g} \geqslant 2^{n}+2
$$


This estimate allows to control the effect of the possible boundaries $\hat{G}$ and $\hat{G}^{\prime}$ on the total $\bar{d}$-distance.

Now, let $\rho=\min _{j} \bar{d}\left(\bar{G}_{i, j}\left(T_{u}, P(\tau)\right), \bar{G}_{i, j}\left(T_{v}^{g}, P^{g}\right)\right)$. If $\rho \geqslant \frac{1}{8} \frac{1-4 \epsilon}{2 \int g}$, then the proposition obtains. Otherwise, let $j_{0}$ be an indice realizing this minimum. The proportion of segments $\bar{G}_{i, j}$ not in $F_{0}$ and such that $\bar{G}_{i, j}$ and $\bar{G}_{i, j_{0}}$ have a different a $n$-type is more than $1-2 / N(n)$. For $n$ sufficiently large, we get, by applying estimates (14) and (17):

$$
\begin{gathered}
\bar{d}\left(\bar{G}_{i}\left(T_{v}^{g}, P^{g}\right), \bar{G}_{i}\left(T_{u}, P(\tau)\right)\right) \\
\geqslant\left(1-\epsilon^{\prime}\right)\left(1-1 / 2^{n}\right) \frac{1}{\bar{w}-2-\left|F_{0}\right|} \sum_{j=2, \bar{G}_{i, j} \notin F_{0}}^{\bar{w}-1} \bar{d}\left(\bar{G}_{i, j}\left(T_{v}^{g}, P^{g}\right), \bar{G}_{i, j}\left(T_{u}, P(\tau)\right)\right) \\
\geqslant\left(1-\epsilon^{\prime}\right)\left(1-1 / 2^{n}\right) \frac{1}{\bar{w}-2-\left|F_{0}\right|} \sum_{j=2, \bar{G}_{i, j} \notin F_{0}}^{\bar{w}-1} \bar{f}\left(\bar{G}_{i, j}\left(T_{v}^{g}, P^{g}\right), \bar{G}_{i, j_{0}}\left(T_{v}^{g}, P^{g}\right)\right) \\
-\bar{f}\left(\bar{G}_{i, j_{0}}\left(T_{v}^{g}, P^{g}\right), \bar{G}_{i, j_{0}}\left(T_{u}, P(\tau)\right)\right)-\bar{f}\left(\bar{G}_{i, j_{0}}\left(T_{u}, P(\tau)\right), \bar{G}_{i, j}\left(T_{u}, P(\tau)\right)\right) \\
\geqslant\left(1-\epsilon^{\prime}\right)\left(1-1 / 2^{n}\right)\left(\left((1-2 / N(n))\left(\frac{1-4 \epsilon}{2 \int g}\right)-\frac{1}{8}\left(\frac{1-4 \epsilon}{2 \int g}\right)-\left(1+1 / 2^{n}\right) \frac{1}{2^{n+2}}\right)\right. \\
\geqslant\left(1-\epsilon^{\prime}\right) \frac{1-4 \epsilon}{8 \int g}
\end{gathered}
$$

The case $q_{n, l_{1}}<q_{n, l_{1}^{\prime}}$ is analogous: we decompose $G_{i}\left(T_{v}^{g}, P^{g}\right)$ into segments of complete cycles (instead of segments of the same type), and we decompose $G_{i}\left(T_{u}, P(\tau)\right)$ into segments of the same type (instead of segments of complete cycles), and we proceed in the same way.

Proof of lemma 4.9. We decompose the $\left(T_{v}^{g}, P^{g}\right)$-trajectory of $(x, i)$ into

$$
\tilde{a}_{n+2,0, \mathrm{eff}} \tilde{a}_{n+2,1, \mathrm{eff}} \cdots \tilde{t}_{\cdots} \ldots
$$

$\tilde{a}_{n+2, i \text {,eff }}$ denotes the $i^{\text {th }}$ word. Its $n+2$-type is $i_{1}\left(i_{1} \in\{0, \ldots, N(n+2)-1\}\right)$. Let $\tilde{B}_{n+2, i}$ be the set of indices such that $\tilde{B}_{n+2, i}\left(T_{v}^{g}, P^{g}\right)=\tilde{a}_{n+2, i, \text { eff }}$.

By lemma 4.3, for $n$ sufficiently large, the set of indices of $\tilde{B}_{n+2, i}$ which does not belong to a word $\tilde{a}_{n+1, j, \mathrm{eff}}$ that satisfies:

$$
\left|\frac{\left|\tilde{a}_{n+1, j, \mathrm{eff}}\right|}{\left|a_{n+1, j, \mathrm{eff}}\right|}-\int g\right| \leqslant \epsilon^{\prime} / 2
$$

has a density of at most $\epsilon^{\prime} / 2$. Thus, the density of indices that we consider is $1-\epsilon^{\prime} / 2$. 


$$
a_{n+1, l} r(n+1, j 1)
$$

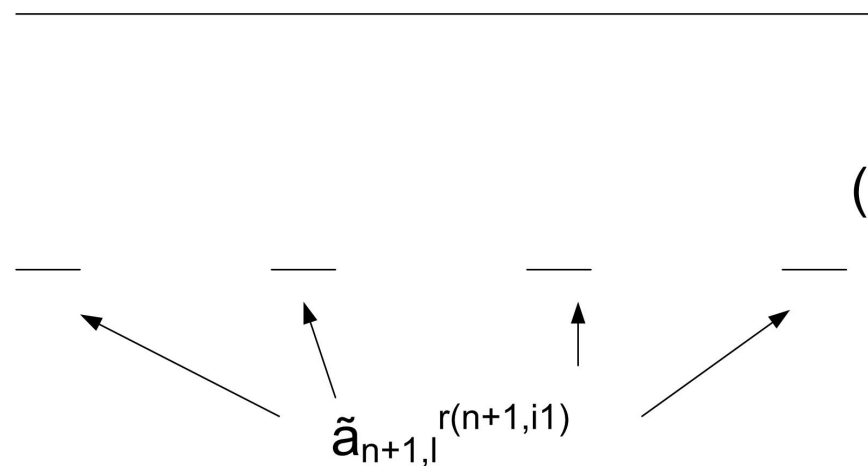

$\left(T_{v}{ }^{g}, P^{g}\right)$

Figure 10: Overlap of a $\left(T_{u}, P\right)$ and $\left(T_{v}^{g}, P^{g}\right)$-trajectories when $r_{n+1, j_{1}}>r_{n+1, i_{1}}$. Turbulences are not represented for simplification.

On $\tilde{B}_{n+2, i}$, which has a $\left(T_{v}^{g}, P^{g}\right)$-trajectory of $n+2$-type $i_{1}$, we distinguish different segments according to the $n+2$-type $j_{1}$ of the $\left(T_{u}, P\right)$-trajectory. We distinguish the cases $j_{1}<i_{1}, j_{1}>i_{1}$ and $j_{1}=i_{1}\left(j_{1} \in\{0, \ldots, N(n+2)-1\}\right)$.

We suppose $j_{1}<i_{1}$ (i.e. $r_{n+1, j_{1}}>r_{n+1, i_{1}}$ ). Let $\tilde{B}_{n+2, i, j}$ be a set of indices such that $\tilde{B}_{n+2, i, j}\left(T_{u}, P\right)$ is a segment of $r_{n+1, j_{1}}$ words of the same $n+1$-type $l$ (modulo turbulences from $A_{n+2}$ and from the effect of the cyclic permutation), i.e. is of the form

$$
\tilde{B}_{n+2, i, j}\left(T_{u}, P\right)=\underbrace{a_{n+1, l, \mathrm{eff}} \cdots a_{n+1, l, \mathrm{eff}} t_{n+1, l, \mathrm{eff}} \cdots a_{n+1, l, \mathrm{eff}}}_{q_{n+1} r_{n+1, j_{1}}=\frac{q_{n+2}}{a_{(n+1) q_{n+1, j_{1}}}} \text { letters }}
$$

Since, for $n$ sufficiently large, $\int g-\epsilon^{\prime} / 2 \geqslant 2^{n} r_{n+1, i_{1}} / r_{n+1, j_{1}}$, then $\tilde{B}_{n+2, i, j}$ contains

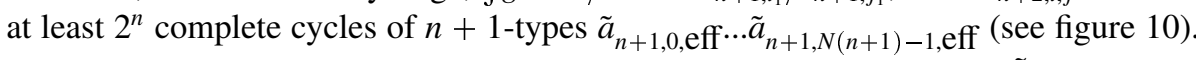
Therefore, the density of $\tilde{a}_{n+1, l, \text { eff }}$ words (i.e. words of $n+1$-type $l$ ) in $\tilde{B}_{n+2, i, j}$ is less than $2 / N(n+1)+1 / 2^{n}$.

We neglected turb $\left(A_{n+1}\right)$ (and cyclic permutation effect), so we need to add an error of density at most $3 \epsilon_{n+1} N_{n+1} q_{n, N(n+1)}$.

If $j_{1}>i_{1}$ (i.e. $r_{n+1, j_{1}}<r_{n+1, i_{1}}$ ), then except maybe on boundaries, there is another segment of indices $\widetilde{B}_{n+2, i, k}$ such that $\tilde{B}_{n+2, i, k}\left(T_{v}^{g}, P^{g}\right)$ is a segment of $r_{n+1, j_{1}}$ words of the same $n+1$-type $l$, i.e. of the form

$$
\tilde{B}_{n+2, i, k}\left(T_{v}^{g}, P^{g}\right)=\underbrace{\tilde{a}_{n+1, l, \text { eff }} \tilde{t}_{\cdots} \ldots \tilde{a}_{n+1, l, \text { effers }}}_{\geqslant q_{n+1} r_{n+1, i,}}
$$

There are more than $q_{n+1} r_{n+1, i_{1}}$ letters because $\int g-\epsilon^{\prime}>1$.

Since $\int g+\epsilon^{\prime} / 2 \leqslant r_{n+1, j_{1}} /\left(2^{n} r_{n+1, i_{1}}\right)$, then $\tilde{B}_{n+2, i, l}\left(T_{u}, P\right)$ contains at least $2^{n}$ com-

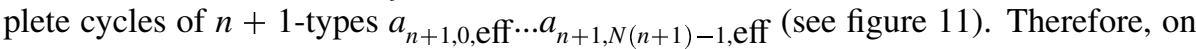
this segment, the $n+1$-types of $\left(T_{v}^{g}, P^{g}\right)$ and $\left(T_{u}, P\right)$ coincide on a set of density at most $2 / N(n+1)+1 / 2^{n}$.

Again, we add an error of density at most $3 \epsilon_{n+1} N_{n+1} q_{n, N(n+1)}$, due to $\operatorname{turb}\left(A_{n+1}\right)$ and cyclic permutation effect. 


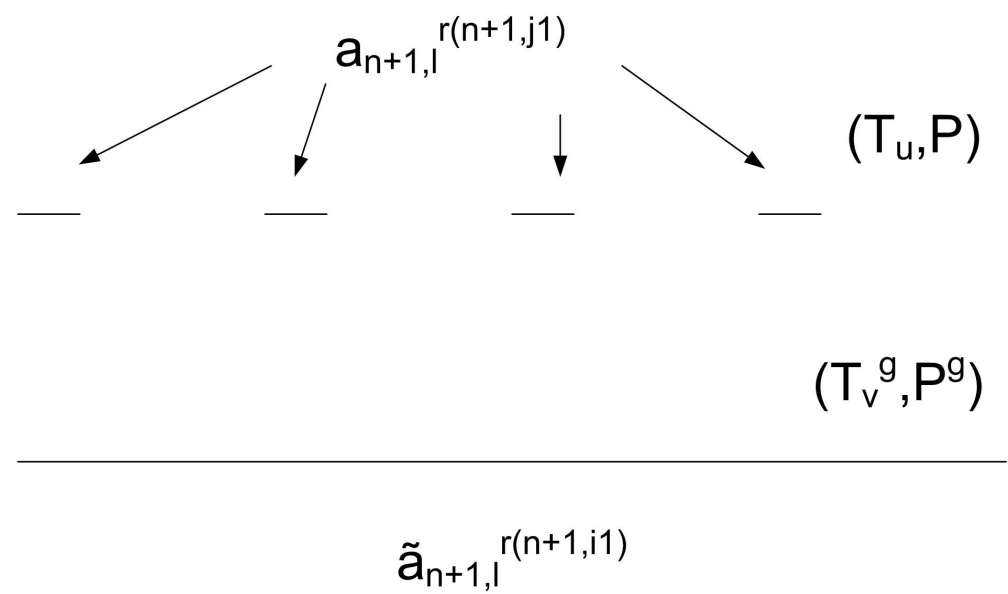

Figure 11: Overlap of a $\left(T_{u}, P\right)$ and $\left(T_{v}^{g}, P^{g}\right)$-trajectories when $r_{n+1, j_{1}}<<r_{n+1, i_{1}}$.

Since $N(n+1)>\int g+\epsilon^{\prime}$ for $n$ sufficiently large, the case $j_{1}=i_{1}$ occurs at most once on every set of indices where $\left(T_{v}^{g}, P^{g}\right)$ is of the form (see figure 12):

$$
\underbrace{\tilde{a}_{n+1, l, \mathrm{eff}} \tilde{t} \ldots \tilde{a}_{n+1, l, \mathrm{eff}}}_{\geqslant q_{n+1} r_{n+1, i_{1}} \text { letters }}
$$

Therefore, there exists $n_{0}$ such that for any $n \geqslant n_{0}$ the density of indices on which the $n+1$-types of the $\left(T_{v}^{g}, P^{g}\right)$ and $\left(T_{u}, P\right)$-trajectories coincide is less than $\epsilon^{\prime}+1 / \int g$.

The proof works because for $n$ sufficiently large, scales for $\left(T_{v}^{g}, P^{g}\right)$ and $\left(T_{u}, P\right)$ are either extremely different, or equal. A more problematic case would be if those scales were different but comparable, e.g. if $r_{n+1, i_{1}}\left|\tilde{a}_{n+1,1}\right| \simeq r_{n+1, j_{1}}\left|a_{n+1,1}\right|$ (see figure 13).

\subsection{Even equivalence}

We show that if $u_{n} \neq v_{n}$ infinitely often, then $T_{u}$ and $T_{v}$ are not evenly equivalent. We apply the proposition [12, p.92]:

Proposition 4.11. If $(S, P)$ and $(T, Q)$ are evenly equivalent, then for any $\tau>0$, there is $K(\tau)>0$ and $P(\tau) \subset \bigvee_{i=-K(\tau)}^{K(\tau)} T^{i}(Q)$ such that $\bar{f}((S, P),(T, P(\tau)))<\tau$.

We contradict this proposition with $\tau=(1-4 \epsilon) / 200$. Indeed, we show:

Proposition 4.12. Let $x, y$ two points in $E_{n l b}$ and $P(\tau) \subset \bigvee_{i=-K(\tau)}^{K(\tau)} T_{u}^{i}(P)$. Then

$$
\liminf _{m \rightarrow+\infty} \bar{f}\left(a\left(T_{u}, P(\tau), m, x\right), a\left(T_{v}, P, m, y\right)\right) \geqslant \frac{1-4 \epsilon}{128}
$$

Proof. The scheme of the proof is not sensibly different from the scheme of the proof of proposition 4.7: we decompose successively the trajectories from scale $n+2$ to scale 


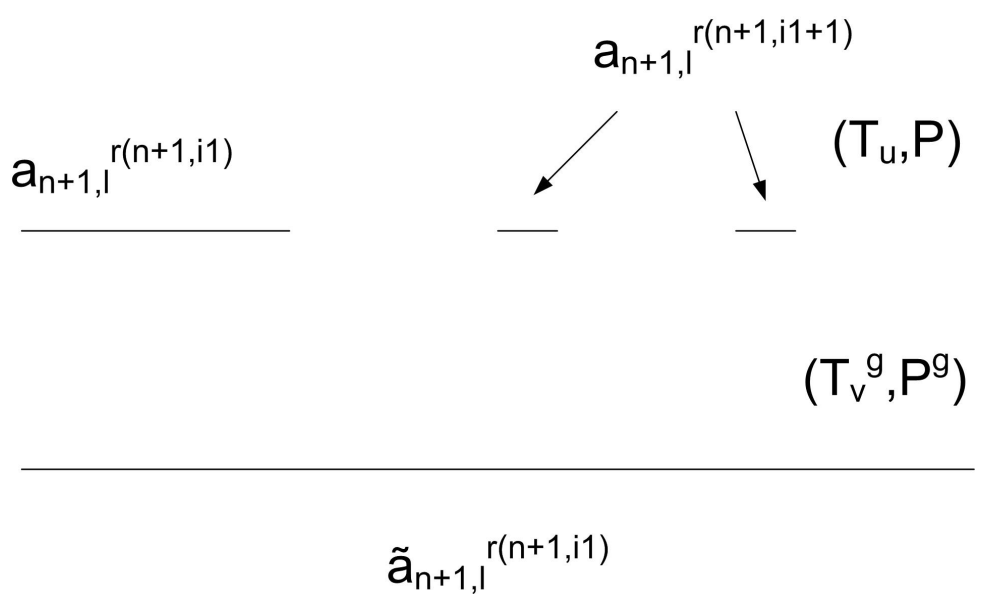

Figure 12: Overlap of a $\left(T_{u}, P\right)$ and $\left(T_{v}^{g}, P^{g}\right)$-trajectories when $r_{n+1, j_{1}}=r_{n+1, i_{1}}$. In the next segment, $r_{n+1, j_{1}}=r_{n+1, i_{1}+1}<<r_{n+1, i_{1}}$

$n$, but along a maximal match, because we are working with $\bar{f}$ (whereas in proposition 4.7, we decomposed along identical ranks of indices, because we were working with $\bar{d}$ ). For $u_{n+1} \neq v_{n+1}$, the orders of complete cycles of the $\left(T_{u}, P\right)$ and $\left(T_{v}, P\right)$-trajectories are the reverse of each other (see figure 15). It implies that in most cases, a $\left(T_{u}, P\right)$ segment of a given $n+1$-type must be matched with a $\left(T_{v}, P\right)$-segment that has a different $n+1$-type. Here, the "reverse orders of cycles" separate trajectories for $\bar{f}$ in the same way as the expansion of trajectories by $g$ separated trajectories for $\bar{d}$ in proposition 4.7 (see lemma 4.9).

In this case, the fit of their match is small, because repetitions of the words $a_{n, i, \mathrm{eff}}$ in the $\left(T_{v}, P\right)$-trajectory and of the words $\check{a}_{n, i, \text { eff }}$ in the $\left(T_{u}, P(\tau)\right)$-trajectory have different periodicities (the periodicity of repetitions of $\breve{a}_{n, i, \mathrm{eff}}$ in the $\left(T_{u}, P(\tau)\right)$-trajectory is the same as the periodicity of repetitions of $a_{n, i, \mathrm{eff}}$ in the $\left(T_{u}, P\right)$-trajectory, because $\left.K(\tau)<q_{n} / 2\right)$.

Turbulences remain packed in rare locations, and do not sensibly affect $\bar{f}$. Thus, we can conclude as in proposition 4.7 .

Let $n$ be sufficiently large such that $q_{n} \geqslant 2^{n+4}(2 K(\tau))$, and such that $u_{n+1} \neq v_{n+1}$ (e.g. $u_{n+1}=0, v_{n+1}=1$ ). Let $m=2^{n} q_{n+2}$. Let $\alpha=1-3 \epsilon$.

If $\bar{f}\left(a\left(T_{u}, P(\tau), m, x\right), a\left(T_{v}, P, m, y\right)\right) \geqslant \frac{1-3 \epsilon}{64}$ then we obtain the proposition. Otherwise, let $\pi: a\left(T_{v}, P, m, y\right) \rightarrow a\left(T_{u}, P(\tau), m, x\right)$ a match minimizing the $\bar{f}$-distance. We see it as a match $\pi: a\left(T_{v}, P, m, y\right) \rightarrow a\left(T_{u}, P, m, x\right)$ (we can do this because $\pi$ is a function of $\{1, \ldots, m\}$ into itself). We decompose these two words in $q_{n+2}$-trajectories:

$$
\begin{aligned}
& a\left(T_{u}, P, m, x\right)=\hat{a}_{1} a_{n+2,1, \mathrm{eff}} a_{n+2,2, \mathrm{eff}} \ldots t \ldots \hat{a}_{2} \\
& a\left(T_{v}, P, m, y\right)={\hat{a_{1}^{\prime}}}_{a_{n+2,1, \mathrm{eff}}^{\prime}}^{a_{n+2,2, \mathrm{eff}}^{\prime} \ldots t \ldots \hat{a}_{2}^{\prime}}
\end{aligned}
$$

such that $\left|\widehat{a_{1}}\right|,\left|\widehat{a_{1}^{\prime}}\right|,\left|\hat{a}_{2}\right|,\left|\hat{a_{2}^{\prime}}\right| \leqslant q_{n+2}$, and where $t$ is a turbulence from $A_{n+3}$ (or a cyclic permutation effect). We write $a\left(T_{u}, P, m, x\right)$ and $a\left(T_{v}, P, m, y\right)$ in the form (see figure 14): 


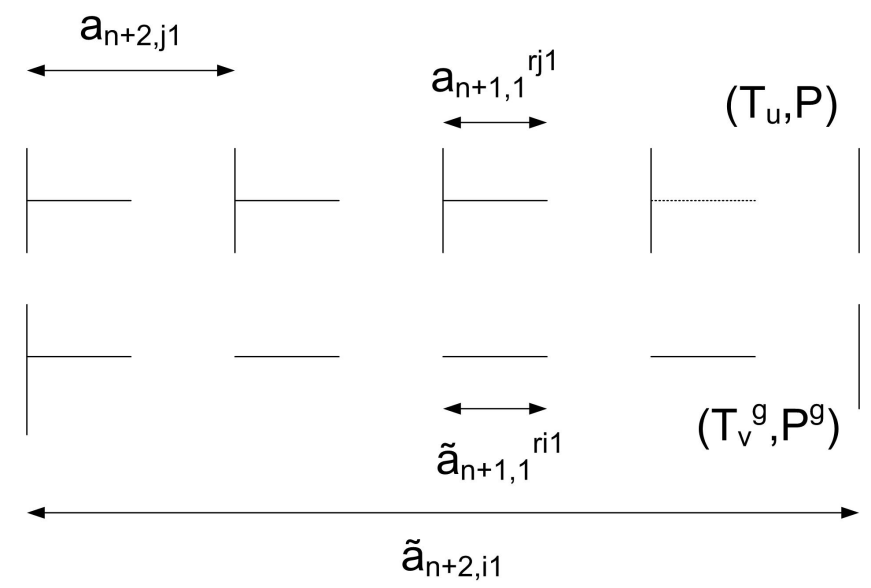

Figure 13: Overlap of a $\left(T_{u}, P\right)$ and $\left(T_{v}^{g}, P^{g}\right)$-trajectories when $r_{n+1, i_{1}}\left|\tilde{a}_{n+1,1}\right|=$ $r_{n+1, j_{1}}\left|a_{n+1,1}\right|$. This case does not happen with our assumptions.

$$
\begin{aligned}
& a\left(T_{v}, P, m, y\right)=G_{1}\left(T_{v}, P, y\right) G_{2}\left(T_{v}, P, y\right) \ldots t \ldots G_{w}\left(T_{v}, P, y\right) \\
& a\left(T_{u}, P, m, x\right)=G_{1}^{\prime}\left(T_{u}, P, x\right) G_{2}^{\prime}\left(T_{u}, P, x\right) \ldots t \ldots G_{w}^{\prime}\left(T_{u}, P, x\right)
\end{aligned}
$$

where $G_{i}, G_{i}^{\prime}$ are sets of indices maximal for the inclusion such that $G_{i} \subset a_{n+2, j_{v}, \text { eff, }}$, $G_{i}^{\prime} \subset a_{n+2, j_{u}}$,eff for some ranks $j_{v}, j_{u}$, and with $G_{i}^{\prime}=\left\{w_{1}, \ldots, w_{2}\right\}$ such that:

$$
\begin{gathered}
w_{1}=1+\max \left\{\pi(u), u<G_{i}, u \in \mathcal{D}(\pi)\right\} \\
w_{2}= \begin{cases}\max \left\{\pi(u), u \in G_{i} \cap \mathcal{D}(\pi)\right\} & \text { if } G_{i} \cap \mathcal{D}(\pi) \neq \varnothing \\
w_{1} & \text { otherwise }\end{cases}
\end{gathered}
$$

In particular, $\left.\pi\left(G_{i}\right) \subset G_{i}^{\prime}\right) . G_{i}^{\prime}$ lies between (but excluding) the rightmost letter matched with a letter left of $G_{i}$ and (including) the rightmost letter identified with a letter to the left and including $G_{i}$. Again, we exclude $A_{n+3}$-turbulences from $G_{i}$, because $|t|>>q_{n+2} \simeq\left|G_{i}\right|$.

If $\bar{f}\left(G_{i}\left(T_{v}, P, y\right), G_{i}^{\prime}\left(T_{u}, P(\tau), x\right)\right) \geqslant \frac{\alpha}{64}$, then we can stop the decomposition here. Otherwise, then

$$
1-1 / 2^{7} \leqslant \frac{\left|G_{i}\right|}{\left|G_{i}^{\prime}\right|} \leqslant 1+1 / 2^{7}
$$

In each $a_{n+2, l, \text { eff }}$, there is at most one $G_{i}$ such that $\left|G_{i}\right| \leqslant q_{n+2} / 2^{n+4}$ (and so $\left|G_{i}^{\prime}\right| \leqslant q_{n+2} / 2^{n+3}$, and there is at most one $G_{i}^{\prime}$ like that in each $a_{n+2, l^{\prime}, \text { eff }}$ ), and therefore, the total density of indices of this kind is at most $1 / 2^{n+2}$. If both $\left|G_{i}\right|$ and $\left|G_{i}^{\prime}\right| \geqslant q_{n+2} / 2^{n+3} \geqslant 2^{n+2} q_{n+1} N(n+1)$, we write:

$$
G_{i}=\widehat{G_{1}} G_{i, 1} \ldots G_{i, r} \widehat{G_{2}}
$$

with $r \geqslant 2^{n+1}$, such that each $G_{i, j}\left(T_{v}, P, y\right)$ is a complete cycle of $n+1$-types, i.e. $G_{i}\left(T_{v}, P, y\right)$ is of the form (since we assumed $v_{n+1}=1$ ): 


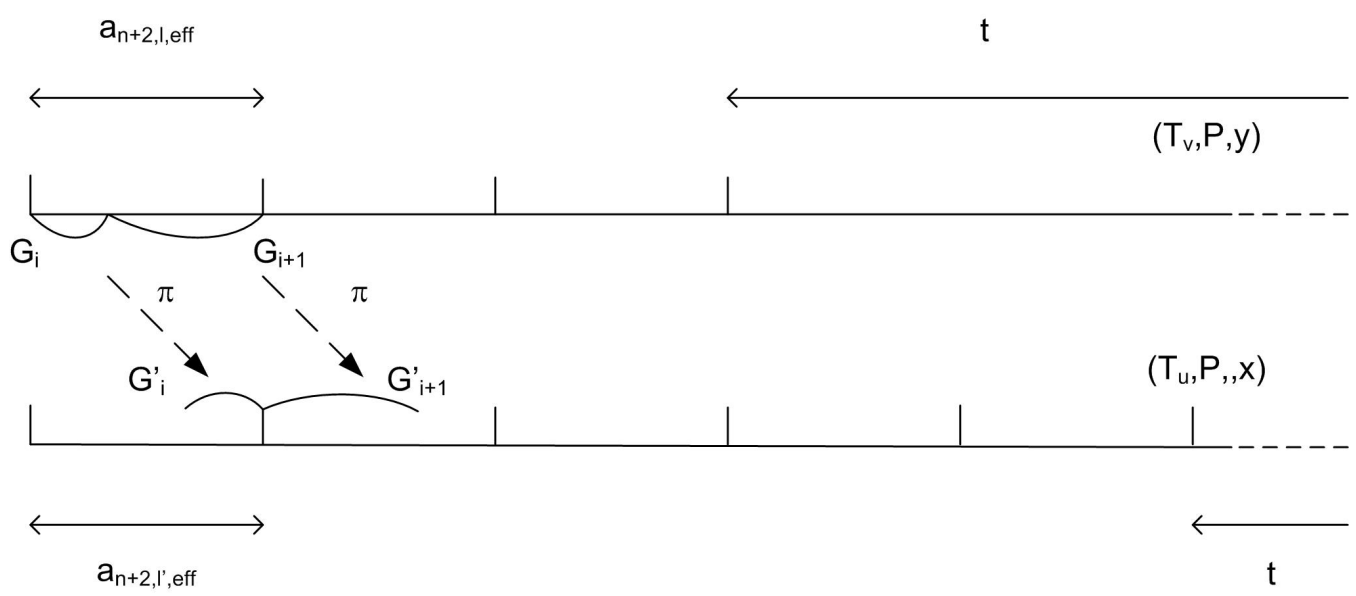

Figure 14: The decomposition of trajectories $a\left(T_{v}, P, m, y\right)$ and $a\left(T_{u}, P, m, x\right)$ in segments $G_{i}$ and $G_{i}^{\prime}$.

$$
G_{i}\left(T_{v}, P, y\right)=\widehat{G_{1}} \underbrace{a_{n+1, N(n+1)-1, \mathrm{eff} \cdots t \ldots a_{n+1,0, \mathrm{eff}}}^{t}}_{G_{i, 1}\left(T_{v}, P, y\right)} \underbrace{a_{n+1, N(n+1)-1, \mathrm{eff}} \ldots t \ldots a_{n+1,0, \mathrm{eff}} t}_{G_{i, 2}\left(T_{v}, P, y\right)} \ldots \widehat{G_{2}}
$$

Moreover, $\left|\widehat{G_{1}}\right|,\left|\widehat{G_{2}}\right| \leqslant N(n+1) q_{n+1}$, and they occupy a density of less than $1 / 2^{n}$. Here, we include $A_{n+2}$-turbulences in $G_{i, j}$, because their density is relatively small. We also write:

$$
G_{i}^{\prime}=\widehat{G_{1}^{\prime}} G_{i, 1}^{\prime} \ldots G_{i, r}^{\prime} \widehat{G_{2}^{\prime}}
$$

such that $G_{i, j}^{\prime}$ corresponds to $G_{i, j}$ by $\pi$, in the same way as $G_{i}^{\prime}$ corresponded to $G_{i}$, (i.e. $G_{i, j}^{\prime}$ lies between the rightmost letter matched with a letter left of $G_{i, j}$ and the rightmost letter identified with a letter to the left and including $G_{i, j}$ ).

If $\bar{f}\left(G_{i, j}\left(T_{v}, P, y\right), G_{i, j}^{\prime}\left(T_{u}, P(\tau), x\right)\right) \geqslant \frac{\alpha}{64}$, then we can stop the decomposition here. Otherwise, then

$$
1-1 / 2^{7} \leqslant \frac{\left|G_{i, j}\right|}{\left|G_{i, j}^{\prime}\right|} \leqslant 1+1 / 2^{7}
$$

We write:

$$
G_{i, j}=G_{i, j, 1} \ldots t . . G_{i, j, s}
$$

and

$$
G_{i, j}^{\prime}=G_{i, j, 1}^{\prime} \ldots t \ldots G_{i, j, s}^{\prime}
$$

where $t$ is a turbulence of $A_{n+2}$ (or a cyclic permutation effect), and $G_{i, j, k}, G_{i, j, k}^{\prime}$ are analogous to $G_{i}$ and $G_{i}^{\prime}$, but at rank $n+1$, i.e. they are sets of indices maximal for the inclusion such that $G_{i, j, k} \subset a_{n+1, k_{v}, \text { eff }}, G_{i, j, k}^{\prime} \subset a_{n+1, k_{u}}$,eff for some ranks $k_{v}, k_{u}$, and $G_{i, j, k}^{\prime}$ lies between the rightmost letter matched with a letter left of $G_{i, j, k}$ and the rightmost letter identified with a letter to the left and including $G_{i, j, k}$. 
If $\bar{f}\left(G_{i, j, k}\left(T_{v}, P, y\right), G_{i, j, k}^{\prime}\left(T_{u}, P(\tau), x\right)\right) \geqslant \frac{\alpha}{64}$, then we can stop the decomposition here. Otherwise, then

$$
1-1 / 2^{7} \leqslant \frac{\left|G_{i, j, k}\right|}{\left|G_{i, j, k}^{\prime}\right|} \leqslant 1+1 / 2^{7}
$$

If either $\left|G_{i, j, k}\right| \leqslant q_{n+1} / 2^{n+3}$ or $\left|G_{i, j, k}^{\prime}\right| \leqslant q_{n+1} / 2^{n+3}$, we can neglect both.

Otherwise, let $k_{1}$ denotes the $n+1$-type of $a_{n+1, k_{v}^{\prime}, \text { eff }} \supset G_{i, j, k}\left(k_{1} \in\{0, \ldots, N(n+\right.$ $1)-1\}$. Since $q_{n+1} / 2^{n+3} \geqslant 2^{n} r_{n, k_{1}} N(n) q_{n}$, then $G_{i, j, k}\left(T_{v}, P, y\right)$ contains $2^{n}$ complete cycles of $n$-types. We can write:

$$
G_{i, j, k}=\hat{a}_{1} a_{n, 0, \mathrm{eff}} \ldots a_{n, 1, \mathrm{eff}} t \ldots a_{n, N(n)-1, \mathrm{eff}} \widehat{a_{2}}
$$

where $\left|\hat{a}_{1}\right|,\left|\hat{a}_{2}\right| \leqslant N(n) q_{n}$, and $\hat{a}_{1}, \hat{a}_{2}$ occupy a density of less than $1 / 2^{n}$.

Let $k_{2}$ be the $n+1$-type of $a_{n+1, k_{u}}$,eff $\supset G_{i, j, k}^{\prime}$. We observe that any match between the words $12 \ldots N 12 \ldots N 12 \ldots N$ and $N \ldots 21 N \ldots 21 N \ldots 21$ (same word repeated $p$ times) has a fit smaller than $\frac{2 p-1}{p} \frac{1}{N} \leqslant 3 / N$, where $N$ is the number of types (see figure 15). Therefore, $G_{i, j, k}\left(T_{v}, P, y\right)$ and $G_{i, j, k}^{\prime}\left(T_{u}, P, x\right)$ have the same type in only a fraction $3 / N(n+1)$ of cases, which makes a density of indices of less than $4 / N(n+1)$.

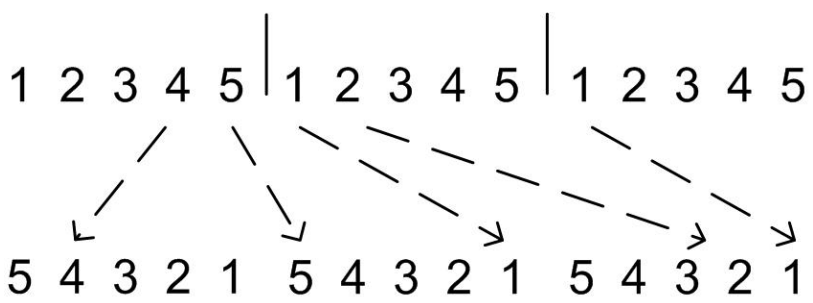

Figure 15: The $\bar{f}$-distance between these two words is large.

If $k_{1}>k_{2}$, then $r_{n, k_{1}}<r_{n, k_{2}}$ and we proceed as in proposition 4.7. We can write:

$$
G_{i, j, k}^{\prime}=\widehat{a}_{3} G_{i, j, k, 1}^{\prime} \ldots G_{i, j, k, L}^{\prime} \widehat{a}_{4}
$$

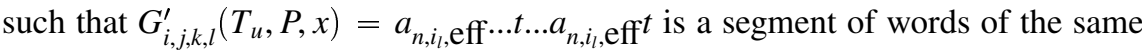
$n$-type $i_{l}$. $G_{i, j, k, l}^{\prime}$ corresponds by $\pi$ to $G_{i, j, k, l}$ (as before).

If $\bar{f}\left(G_{i, j, k, l}\left(T_{v}, P, y\right), G_{i, j, k, l}^{\prime}\left(T_{u}, P(\tau), x\right)\right) \geqslant \frac{\alpha}{64}$, then we can stop the work with this segment here. Otherwise, then

$$
1-1 / 2^{7} \leqslant \frac{\left|G_{i, j, k, l}\right|}{\left|G_{i, j, k, l}^{\prime}\right|} \leqslant 1+1 / 2^{7}
$$

$G_{i, j, k, l}\left(T_{v}, P, y\right)$ contains at least $2^{n}$ complete cycles of $n$-types (with turbulences), because $2^{n+1} r_{n, k_{1}}<r_{n, k_{2}}$.

At $i, j, k$ fixed, the $G_{i, j, k, l}^{\prime}\left(T_{u}, P, x\right)$, when $l$ varies, have the same $n$-type in a proportion of less than $2 / N(n)$. Since $K(\tau) \leqslant q_{n} / 2$, and the $a_{n, i_{l} \text {,eff }}$ are the same (turbulences coming from $B_{n}$ are located at the same place throughout every $\left.a_{n, i_{l}, \text { eff }}\right)$, then the $G_{i, j, k, l}^{\prime}\left(T_{u}, P(\tau), x\right)$ also have the same $n$-type in a proportion of less than $2 / N(n)$. If $l_{1} \neq l_{2}$, then because of $\operatorname{turb}\left(A_{n+1}\right)$ (and cyclic permutation effect),

$$
\bar{f}\left(G_{i, j, k, l_{1}}^{\prime}\left(T_{u}, P(\tau), x\right), G_{i, j, k, l_{2}}^{\prime}\left(T_{u}, P(\tau), x\right)\right) \geqslant \alpha-4(2 K(\tau)+1) \epsilon_{n+1} N_{n+1} q_{n, N(n+1)}
$$


On the other hand, since $G_{i, j, k, l}\left(T_{v}, P, y\right)$ contains at least $2^{n}$ complete cycles of $n$-types (with turbulences), then for any $l_{1}, l_{2}$,

$$
\bar{f}\left(G_{i, j, k, l_{1}}\left(T_{v}, P, y\right), G_{i, j, k, l_{2}}\left(T_{v}, P, y\right)\right) \leqslant \frac{1}{2^{n}}+4 \epsilon_{n+1} N_{n+1} q_{n, N(n+1)}
$$

If $k_{1}<k_{2}$, the proof is analogous.

Let $\rho_{0}=\min _{l} \bar{f}\left(G_{i, j, k, l}^{\prime}\left(T_{u}, P(\tau), x\right), G_{i, j, k, l}\left(T_{v}, P, y\right)\right)$ and $l_{0}$ an indice realizing this minimum. If $\rho_{0}>\alpha / 128$, we can stop here. Otherwise, by applying estimates (18) and (19), and by taking into account boundary effects, and for $n$ sufficiently large such that $a_{n+1} \neq b_{n+1}$ :

$$
\begin{gathered}
\bar{f}\left(G_{i, j, k}^{\prime}\left(T_{u}, P(\tau), x\right), G_{i, j, k}\left(T_{v}, P, y\right)\right) \geqslant\left(1-1 / 2^{n}\right) \frac{1}{L} \sum_{l=1}^{L} \bar{f}\left(G_{i, j, k, l}^{\prime}\left(T_{u}, P(\tau), x\right), G_{i, j, k, l}\left(T_{v}, P, y\right)\right) \\
\geqslant\left(1-1 / 2^{n}\right) \frac{1}{L} \sum_{l=1}^{L} \bar{f}\left(G_{i, j, k, l}^{\prime}\left(T_{u}, P(\tau), x\right), G_{i, j, k, l_{0}}^{\prime}\left(T_{u}, P(\tau), x\right)\right) \\
-\bar{f}\left(G_{i, j, k, l_{0}}^{\prime}\left(T_{u}, P(\tau), x\right), G_{i, j, k, l_{0}}\left(T_{v}, P, y\right)\right)-\bar{f}\left(G_{i, j, k, l_{0}}\left(T_{v}, P, y\right), G_{i, j, k, l}\left(T_{v}, P, y\right)\right) \\
\geqslant \frac{1-4 \epsilon}{128}
\end{gathered}
$$

\section{References}

[1] D.V. Anosov and A.B. Katok. New examples in smooth ergodic theory. Ergodic diffeomorphisms. Trans. Moscow Math. Soc, 23(1):35, 1970.

[2] P. Arnoux, D.S. Ornstein, and B. Weiss. Cutting and stacking, interval exchanges and geometric models. Israel journal of mathematics, 50(1):160-168, 1985.

[3] M. Benhenda. Non-standard couples of angles of rotations. http://hal. archives-ouvertes. fr/hal-00669028, February 2012.

[4] M. Benhenda. Non-standard smooth realization of translations on the torus. http: //hal . archives-ouvertes . fr/hal-00669027, February 2012.

[5] M. Brin, J. Feldman, and A.B. Katok. Bernoulli diffeomorphisms and group extensions of dynamical systems with non-zero characteristic exponents. Ann. Math, 113:159-179, 1981.

[6] B. Fayad, M. Saprykina, and A. Windsor. Non-standard smooth realizations of Liouville rotations. Ergodic Theory and Dynamical Systems, 27(06):1803-1818, 2007.

[7] J. Feldman. New K-automorphisms and a problem of Kakutani. Israel Journal of Mathematics, 24(1):16-38, 1976.

[8] A.B. Katok. Monotone equivalence in ergodic theory. Mathematics of the USSRIzvestiya, 11:99, 1977. 
[9] A.B. Katok. Smooth non-Bernoulli K-automorphisms. Inventiones Mathematicae, 61(3):291-299, 1980.

[10] W. Krieger. On entropy and generators of measure-preserving transformations. Trans. Amer. Math. Soc, 149:453-464, 1970.

[11] D.S. Ornstein and B. Weiss. Statistical properties of chaotic systems. Bulletin of the American Mathematical Society, 24(1):11-116, 1991.

[12] Rudolph D. Ornstein, D.S. and B. Weiss. Equivalence of measure-preserving transformations. Mem. Amer. Math. Soc., 37(262), 1982.

[13] M. Ratner. The Cartesian square of the horocycle flow is not loosely Bernoulli. Israel Journal of Mathematics, 34(1):72-96, 1979.

[14] D.J. Rudolph. Asymptotically Brownian skew products give non-loosely Bernoulli K-automorphisms. Inventiones mathematicae, 91(1):105-128, 1988.

[15] J.P. Thouvenot. Entropy, isomorphism and equivalence in ergodic theory. Handbook of dynamical systems, 1:205-238, 2002. 